\title{
Perturbações Periódicas de Ciclos Heteroclínicos Infinitos de Campos Vetoriais Polinomiais Planares
}

\author{
Marcelo Messias
}

TESE APRESENTADA AO

INSTITUTO DE MATEMÁTICA E ESTATÍSTICA

DA UNIVERSIDADE DE SÃO PAULO

PARA OBTENÇÃO DO GRAU DE

DOUTOR EM MATEMÁTICA APLICADA

Área de Concentração: Equações Diferenciais e Sistemas Dinâmicos

Orientador: Prof. Dr. Jorge Manuel Sotomayor Tello

São Paulo - Junho de 2000

Durante a elaboração deste trabalho o autor recebeu auxilio financeiro da CAPES - Convênio PICDT 045/97. 


\title{
Perturbações Periódicas de Ciclos Heteroclínicos Infinitos de Campos Vetoriais Polinomiais Planares
}

\author{
Este exemplar corresponde à redação \\ final da tese, devidamente corrigida, \\ defendida por Marcelo Messias e \\ aprovada pela comissão julgadora.
}

São Paulo, 15 de Junho de 2000.

Banca examinadora:

- Prof. Dr. Jorge Manuel Sotomayor Tello (Orientador) - IME-USP

- Prof. Dr. Clodoaldo Grotta Ragazzo - IME-USP

- Prof. Dr. Plácido Zoega Táboas - ICMC - USP

- Prof. Dr. Hildebrando Munhoz Rodrigues - ICMC - USP

- Prof. Dr. Ronaldo Alves Garcia - UFG 
Para minhas meninas

Iracimara e Sofia 


\section{AGRADECIMENTOS}

Agradeço a Deus, por tudo.

Em primeiro lugar, sou imensamente grato ao Prof. Jorge Sotomayor, pela indicação do problema aqui estudado, pela orientação e incentivo a mim dedicados ao longo destes quatro anos do Curso de Doutorado, através de sua grande experiência e admirável conhecimento matemático.

Agradeço de coração aos meus amigos e familiares, especialmente minha esposa Iracimara, pela paciência, apoio e incentivo durante estes anos de estudo.

Sou grato aos colegas e companheiros de doutoramento no IME-USP, especialmente ao Roberto, a Ana Lúcia e ao Luiz Fernando, por todos os momentos que passamos juntos, na busca de um objetivo comum. Sou muito grato também ao amigo Orlando Stanley, pelo apoio, pelas conversas e pela sincera e fraterna amizade de sempre.

Durante a elaboração do trabalho obtive apoio financeiro da CAPES - Convênio PICDT-UNESP 045/97, pelo qual sou imensamente grato. Procurarei trabalhar para que o apoio financeiro que recebi do Estado se reverta em benefícios à sociedade.

Sou grato também aos colegas do Departamento de Matemática da FCT-UNESP, que propiciaram o afastamento integral de minhas atividades docentes durante a maior parte do doutoramento, sem o qual seria inviável a realização deste trabalho.

Finalmente, agradeço ao pessoal do Instituto de Matemática e Estatística da Universidade de São Paulo, particularmente do Departamento de Matemática Aplicada, onde foi desenvolvido o trabalho.

Sinceramente sou,

Marcelo Messias. 


\section{RESUMO}

Neste trabalho são estudadas perturbações periódicas, a dois parâmetros, de campos vetoriais polinomiais planares, que possuem um ciclo heteroclínico infinito, que consiste de uma solução ilimitada, que conecta dois pontos de sela "no infinito". O estudo global, envolvendo o infinito, é elaborado via compactificação de Poincaré. Mostra-se que, para certos tipos de perturbações periódicas, existem curvas diferenciáveis, contidas em uma vizinhança da origem no espaço de parâmetros, para as quais o sistema perturbado apresenta tangências quadráticas entre as variedades invariantes locais de órbitas periódicas no infinito. Tais curvas dividem uma vizinhança da origem, no espaço de parâmetros, em quatro regiões disjuntas: em duas delas, as variedades invariantes mencionadas acima, localizadas ao longo da órbita de tangência heteroclínica, se intersectam transversalmente; nas outras duas, a intersecção entre tais variedades é vazia.

A existência de instersecções transversais entre as variedades invariantes das órbitas periódicas no infinito implica, via o Teorema de Birkhoff-Smale, em um complexo comportamento dinâmico das soluções do sistema perturbado, na parte finita do plano.

Analisa-se também o caso em que existe um anel de órbitas periódicas de grande amplitude que se acumula nos ciclos heteroclínicos infinitos. Mostra-se que, para determinados valores dos parâmetros, existem subharmônicas de ordem $m$, que são órbitas periódicas de período $m$ do sistema perturbado, que bifurcam de órbitas periódicas ressonantes, contidas no anel que se acumula nos ciclos heteroclínicos infinitos.

Por fim, faz-se uma conexão entre o comportamento limite das bifurcações subharmônicas das órbitas periódicas de grande amplitude, e as bifurcações heteroclínicas dos ciclos heteroclínicos infinitos. 


\begin{abstract}
In this thesis are studied periodic perturbations, depending on two parameters, of planar polynomial vector fields having an infinite heteroclinic cycle, which is a trajectory joining two saddle points at infinity. The global study envolving infinity is performed via the Poincaré compactification. It is shown that for certain types of periodic perturbations, there exist two differentiable curves in the neighborhood of the origin in the parameter space, for which the perturbed system has heteroclinic tangencies between the local stable and unstable manifolds of the hyperbolic periodic orbits at infinity. Also, such curves divide the neighborhood of the origin in the parameter space into four regions: in two of them, the stable and unstable manifolds mentioned above present transverse heteroclinic points, while in the others, the intersection of the manifolds is empty.

The existence of transverse intersection between the invariant manifolds of the periodic orbtis at infinity implies, via the Bikhoff-Smale Theorem, in a complex dynamic behaviour of the solutions of the perturbed system, in a finite part of the phase plane.

An analysis of the case on which there exists an annulus of periodic orbits of large amplitude that accumulate on the infinite heteroclinic cycle is performed. Its shown that, for certain values of parameters, there exist subharmonics of order $m$ (i.e., periodic orbits of period $m$ of the perturbed system), which bifurcate from the ressonant periodic orbits, belonging to the annulus that accumulate on the infinite heteroclinic cycle.

Finally, it is established a connection between the limit behaviour of the subharmonic bifurcations and the heteroclinic bifurcations occurred for the infinite heteroclinic cycles.
\end{abstract}




\section{Sumário}

1 Introdução 3

1.1 Perturbações periódicas . . . . . . . . . . . . . . . . 4

1.2 Campos vetoriais polinomiais no plano . . . . . . . . . . 7

1.3 Principais resultados obtidos . . . . . . . . . . . . . 8

2 Construções e Resultados Preliminares $\quad 15$

2.1 Introdução . . . . . . . . . . . . . . . . . . . . 15

2.2 Compactificação usando coordenadas polares . . . . . . . . . . 16

2.3 Compactificação de perturbações periódicas de campos polinomiais pla-

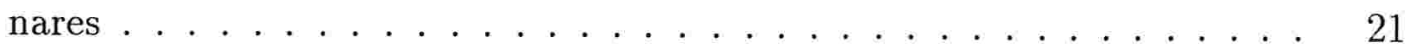

2.4 A função de separação para variedades invariantes de órbitas periódicas no infinito . . . . . . . . . . . . . . . . . . . 24

2.5 A integral de Melnikov-Sotomayor em coordenadas polares . . . . . . 32

3 Perturbações Periódicas de Ciclos Heteroclínicos Infinitos 36

3.1 Caso 1: Campos quadráticos com uma reta invariante . . . . . . . . 36

3.2 Simulações numéricas . . . . . . . . . . . . . . . . . . . . . . 54

3.3 Um resultado mais geral . . . . . . . . . . . . . . . . 61

3.4 Caso 2: Campos polinomiais de grau $n$ com uma reta invariante . . . 65

3.5 Caso 3: Campos polinomiais com uma curva qualquer invariante . . . 66 
3.6 Caso 4: Ciclos singulares semi-infinitos . . . . . . . . . . . . 72

4 Órbitas Periódicas Ressonantes e Bifurcações Subharmônicas $\quad 75$

4.1 Definições e resultados preliminares . . . . . . . . . . . . . . 76

4.1.1 Equações variacionais e bifurcações subharmônicas . . . . . . 79

4.1.2 Bifurcações subharmônicas em um anel regular de órbitas periódicas 82

4.2 Bifurcações subharmônicas de campos polinomiais com uma reta invari-

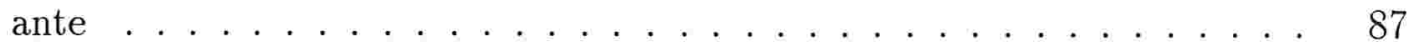

4.3 Estudo do caso quadrático . . . . . . . . . . . . . . . . . . 103

4.4 Comportamento assintótico das bifurcações subharmônicas - caso qua-

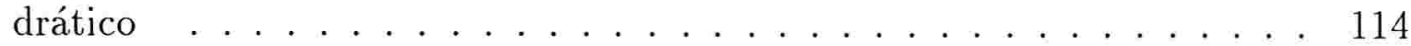

5 Conclusões e Considerações Finais $\quad 122$

Referências Bibliográficas $\quad \mathbf{1 2 5}$ 


\section{Capítulo 1}

\section{Introdução}

O estudo da teoria qualitativa das equações diferenciais ordinárias teve início com o trabalho de H.Poincaré "Sur les courbes définies par une équation differéntielle", publicado em 1881. Desde então, apresentou um desenvolvimento intenso e atualmente é bastante difundido, em virtude de seu interesse teórico e de sua aplicabilidade no estudo de fenômenos naturais em diversas áreas do conhecimento.

Um problema importante desta teoria é o estudo de pequenas perturbações dos sistemas de equações diferenciais (ou campos vetoriais). Neste contexto, busca-se saber se a estrutura topológica das soluções do sistema se altera ou não por tais perturbações. Trata-se de um problema importante no âmbito das aplicações, pois quando se busca modelar fenômenos físicos utilizando-se equações diferenciais, inevitavelmente se cometem erros inerentes ao processo de modelagem. Assim, é importante saber se pequenas alterações do sistema implicam ou não em significativas alterações na estrutura topológica de suas soluções.

Sabe-se que certos tipos de perturbações não só alteram a estrutura topológica das soluções dos sistemas, mas também podem levar ao comportamento caótico destas soluções. Um caso conhecido é o de perturbações periódicas de campos vetoriais que possuem órbitas homoclínicas ou heteroclínicas. 


\subsection{Perturbações periódicas}

Dentre os tipos estudados de perturbações de campos vetoriais no plano estão as perturbações periódicas. Por exemplo, os osciladores lineares e não-lineares perturbados com uma função periódica (oscilações forçadas) são bastante estudados, tanto pelo rico comportamento dinâmico que apresentam quanto pelo fato de serem utilizados como modelos matemáticos de importantes fenômenos da Física e da Engenharia. Alguns dos osciladores forçados mais estudados são o de Duffing, dado por $\ddot{x}=x-x^{3}+\epsilon(\gamma \cos \omega t-\delta y)$, o do pêndulo, $\ddot{x}=-\operatorname{sen} x+\epsilon \cos \omega t$, e o de Van der Pol, $\ddot{x}=-x-\epsilon\left(x^{2}-1\right) y+\epsilon \alpha \cos \omega t$. O plano de fase dos sistemas acima apresenta, para $\epsilon=0$, três tipos importantes de órbitas: periódicas, homoclínicas e heteroclínicas (Figura 1.1).

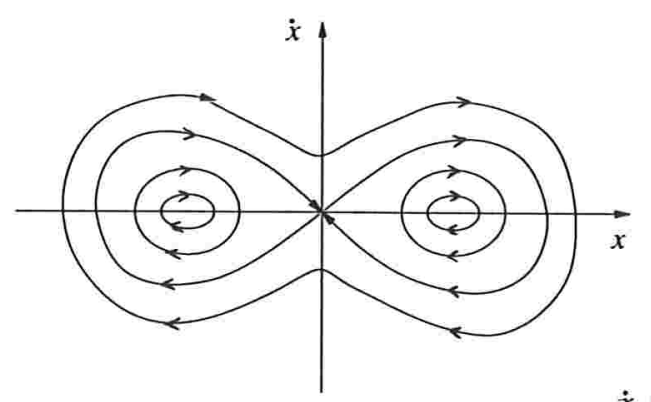

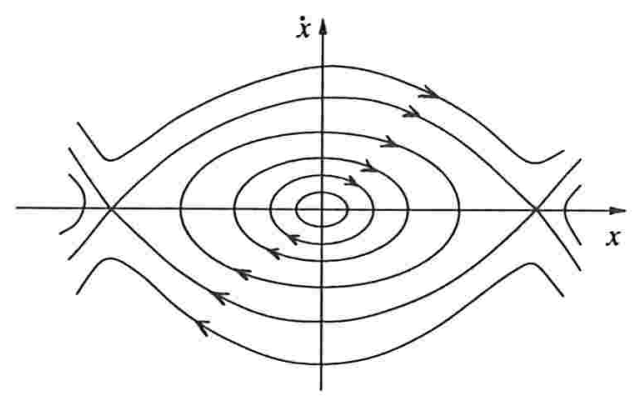

(b)

(a)

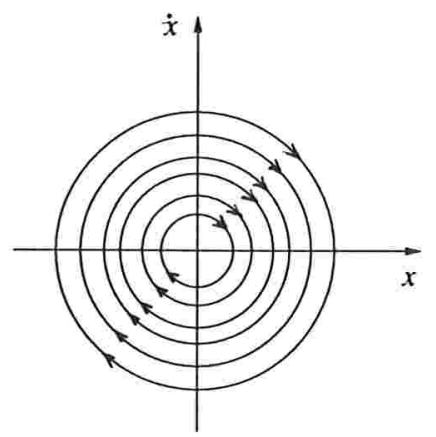

(c)

Figura 1.1: Plano de fase das equações de Duffing (a), do pêndulo (b) e de Van der Pol (c), com $\epsilon=0$.

Em decorrência deste fato, pequenas perturbações periódicas introduzidas nestes 
sistemas, tomando-se $\epsilon \neq 0$ suficientemente pequeno nas equações acima, podem elevar o grau de complexidade das soluções, do ponto de vista dinâmico. Por exemplo, para $\epsilon=0$, as soluções dos sistemas considerados estão contidas no plano de fase $\mathbb{R}^{2}$, enquanto que, para $\epsilon \neq 0$, as soluções dos sistemas perturbados (não-autônomos) estão contidas no espaço de fase $\mathbb{R}^{3}$, ou melhor, considerando-se o caráter periódico da perturbação, no cilindro de fase $\mathbb{R}^{2} \times \mathrm{S}^{1}$. As soluções podem apresentar-se bastante complexas, dependendo dos valores de $\epsilon$ e $\omega$. De fato, pode-se mostrar, no caso do pêndulo e da equação de Duffing, a existência de pontos homoclínicos transversais para a transformação de Poincaré associada aos sistemas perturbados, que leva ao fenômeno de "horseshoe" e a consequente complexidade dinâmica de suas soluções (ver, por exemplo, [15] ou [29]).

Sabe-se, desde Poincaré, que a existência de um ponto homoclínico transversal para um difeomorfismo (neste caso dado pela transformação de Poincaré associada ao sistema perturbado) implica na existência de infinitos pontos homoclínicos transversais. Birkhoff mostrou que todo ponto homoclínico tranversal é limite de pontos periódicos. Mais tarde, Smale mostrou também que a presença de um ponto homoclínico transversal implica a existência de um conjunto de Cantor invariante, contido no conjunto de pontos não-errantes, e que, a partir de um certo número de iterações, o difeomorfismo restrito a esse conjunto é conjugado ao automorfismo "shift" sobre sequências de dois símbolos, herdando assim sua complexidade dinâmica (existência de infinitas órbitas periódicas de todos os períodos, existência de órbitas densas, etc..). Para uma referência sobre estes assuntos, ver, por exemplo, o artigo [22].

Ainda com relação aos sistemas acima, estuda-se o que ocorre com o anel de órbitas periódicas existente pára $\epsilon=0$, em virutde de perturbações periódicas. Neste contexto, mostra-se que, se existe uma órbita periódica contida no anel, cujo período satisfaz uma condição de ressonância com o período da função de perturbação, então, sob certas condições de não-degenerescencia, desta órbita bifurcam soluções periódicas do sistema perturbado [4], [5], [16], [29]. Tais órbitas são chamadas subharmônicas (ver Capítulo 4). 
Resumindo, dado um sistema da forma

$$
\begin{aligned}
& \dot{x}=f(x, y) \\
& \dot{y}=g(x, y)
\end{aligned}
$$

onde $f$ e $g$ são funções de classe $C^{r}$, pode-se considerar os seguintes sistemas perturbados

$$
\begin{aligned}
\dot{x} & =f(x, y)+\epsilon f_{1}(x, y, t) \\
\dot{y} & =g(x, y)+\epsilon g_{1}(x, y, t)
\end{aligned}
$$

onde $\epsilon$ é um parâmetro real, $f_{1}$ e $g_{1}$ são funções periódicas na variável $t$. Tal perturbação pode introduzir (e, de modo geral, introduz) novos fenômenos à dinâmica do sistema, como a existência de atratores estranhos e fenômenos tipo "horseshoe", em adição a pontos de equilíbrio e ciclos-limites, comuns em sistemas planares.

Como o sistema (1.3)-(1.4) está definido em $\mathbb{R}^{2} \times \mathrm{S}^{1}$, a técnica utilizada para seu estudo é a análise da Transformação de Poincaré $P: \Sigma \longrightarrow \Sigma$, definida em uma seção $\Sigma$, transversal ao fluxo do sistema (Capítulo 2). Desse modo, pontos fixos e curvas fechadas invariantes de $P$ correspondem a órbitas periódicas e toros invariantes do sistema perturbado, respectivamente. Além disso, a existência de pontos fixos homoclínicos ou heteroclínicos transversais de $P$ implicam na existência de uma dinâmica tipo "horseshoe" para as soluções do sistema perturbado (conforme Teorema de Birkhoff-Smale [15], [29]).

Campos vetoriais da forma (1.3)-(1.4) foram amplamente estudados, por matemáticos e físicos, para diversas classes de funções $f, g, f_{1}$ e $g_{1}$. Na grande maioria dos trabalhos que aparecem na literatura considera-se o caso em que o sistema não perturbado (1.1)(1.2) está definido em uma região compacta do plano e é hamiltoniano (conservativo), caso em que é comum a ocorrência de órbitas homoclínicas e heteroclínicas, além de anéis de órbitas periódicas. Para uma referência sobre o assunto, pode-se consultar o livro [15], juntamente com a vasta bibliografia ali citada. 
No presente trabalho estuda-se perturbações periódicas da forma (1.3)-(1.4), no caso em que $f$ e $g$ são polinômios de grau $n$ nas variáveis $(x, y), f_{1}$ e $g_{1}$ são funções de classe $C^{k}, k \geq 2$, periódicas de período $T$, na variável $t$. Os sistemas aqui considerados são, em geral, não-hamiltonianos. Algumas das motivações que levaram ao estudo dos sistemas polinomiais são descritas na seção seguinte.

\subsection{Campos vetoriais polinomiais no plano}

Sabe-se que funções de classe $C^{r}$ podem, em geral, ser aproximadas por polinômios de grau n. Assim sendo, o estudo de sistemas da forma (1.3)-(1.4) no caso particular em que as funções $f$ e $g$ são polinômios de grau n é bastante relevante, pois, estudando-se o comportamento das soluções do sistema polinomial, pode-se obter propriedades do sistema no caso em que $f$ e $g$ são funções mais gerais.

Além disso, o estudo de (1.3)-(1.4) existente na literatura é, em geral, elaborado considerando-se regiões compactas do plano, nas quais o campo apresenta órbitas periódicas, homoclínicas e heteroclínicas. Porém, a restrição a regiões compactas apresenta uma certa limitação. Com efeito, o sistema pode apresentar, por exemplo, órbitas periódicas de grande amplitude, que não são detectadas nestas regiões, por maior que elas sejam consideradas. Além disso, considerando-se regiões limitadas não se pode fazer um esboço completo do plano de fase do sistema ou detectar com precisão as regiões onde as soluções são limitadas ou a forma como elas tendem para o infinito. O campo pode apresentar também ciclos singulares infinitos, que são soluções que conectam pontos singulares no infinito (Capítulo 2) e, portanto, não são observadas inteiramente em regiões compactas do plano.

Para o caso dos campos vetoriais polinomiais existe uma técnica que permite o estudo do campo "no infinito": a compactificação, introduzida por H.Poincaré em 1881. Esta técnica permite induzir, a partir de um campo vetorial polinomial no plano e via projeção central, um campo vetorial analítico na esfera $S^{2}$, para o qual o equador $S^{1}$ é 
invariante. Desta forma, os pontos "no infinito" do $\mathbb{R}^{2}$ são representados por pontos do equador $\mathrm{S}^{1} \subset \mathrm{S}^{2}$, e o estudo de propriedades do campo vetorial nas proximidades do infinito é realizado analisando-se o campo induzido na esfera numa vizinhança do equador. Assim, pode-se definir pontos de equilíbrio e órbitas periódicas no infinito, e estudá-los quanto a hiperbolicidade e estabilidade. Pode-se também estudar, via compactificação, as órbitas periódicas de grande amplitude e as conexões de pontos de equilíbrio no infinito, chamados ciclos singulares infinitos (ver Capítulo 2).

Perturbações autônomas de campos polinomiais definidos no plano todo foram estudadas por Paterlini e Sotomayor em [25], artigo que serviu como ponto de partida para o estudo desenvolvido aqui.

No presente trabalho estuda-se certos tipos de perturbações periódicas de campos vetoriais polinomiais do plano. Mais especificamente, utilizando-se a compactificação, considera-se o efeito dessas perturbações nos ciclos singulares infinitos e nas órbitas periódicas de grande amplitude, que não podem ser analisados em regiões limitadas do plano. Analisa-se as alterações dinâmicas que ocorrem com as soluções nas partes compactas do plano, em decorrência destas perturbações periódicas. Com os resultados obtidos pode-se concluir, via o Teorema de Birkhoff-Smale, que certas perturbações periódicas de ciclos singulares infinitos podem levar a existência de horseshoe e, consequentemente, ao comportamento caótico das soluções do sistema perturbado nas partes compactas do plano: é o "caos que surge do infinito"!

\subsection{Principais resultados obtidos}

Considere o seguinte sistema de equações diferenciais ordinárias no $\mathbb{R}^{2}$

$$
\begin{aligned}
& \dot{x}=P(x, y) \\
& \dot{y}=Q(x, y),
\end{aligned}
$$


onde

$$
P(x, y)=\sum_{k=0}^{n} P_{k}(x, y) \quad e \quad Q(x, y)=\sum_{k=0}^{n} Q_{k}(x, y),
$$

com $P_{k}$ e $Q_{k}$ polinômios homogêneos de grau $k$.

Paterlini e Sotomayor [25] estudaram as bifurcações dos campos vetoriais da forma acima, decorrentes de perturbações autônomas nos coeficientes dos polinômios, descrevendo nove tipos diferentes de bifurcações genéricas de codimensão um, envolvendo o "infinito", representado, via compactificação, pelo equador $\mathrm{S}^{1} \subset \mathrm{S}^{2}$.

No presente trabalho considera-se as bifurcações que envolvem o infinito do sistema (1.5)-(1.6), decorrentes de perturbações periódicas da forma

$$
\begin{aligned}
& \dot{x}=P(x, y)+\mu f(\mu, A, \tilde{\omega} t) \\
& \dot{y}=Q(x, y)+\mu g(\mu, A, \tilde{\omega} t)
\end{aligned}
$$

onde $\mu$ e $A$ são parâmetros reais, $f$ e $g$ são funções de classe $C^{k}, k \geq 2$, periódicas de período $T=2 \pi / \tilde{\omega}$, na variável independente $t$, sendo $\tilde{\omega}=\omega\left(\sqrt{x^{2}+y^{2}}\right)^{n-1}$, $\operatorname{com} \omega>0$ constante. Tais sistemas surgem naturalmente no estudo de problemas físicos, como oscilações de sistemas mecânicos e elétricos com termo forçante periódico.

No Capítulo 2 são feitas as construções necessárias ao estudo de (1.7)-(1.8), como a compactificação do sistema, que por ser não-autônomo, induz um campo definido no toro sólido $\mathrm{D}^{2} \times \mathrm{S}^{1}$, e não na esfera $\mathrm{S}^{2}$. São também estabelecidos resultados preliminares importantes, que serão usados, nos Capítulos 3 e 4, na demonstração dos principais resultados do trabalho, os quais descrevemos a seguir.

Suponha que (1.5)-(1.6) possui dois, e somente dois, pontos de sela hiperbólicos simétricos no infinito, ou seja, sua compactificação induz um campo de Poincaré $p(X)$ que possui dois pontos críticos simétricos, $\theta_{1}$ e $\theta_{2}$, no bordo do disco $\mathrm{D}^{2}$ (conforme Capítulo 2). Suponha ainda que tais pontos são conectados por uma solução heteroclínica $\gamma(s)$ (Figura 1.2), correspondente a uma solução $\varphi(t)$ do campo polinomial no plano, contida em uma reta invariante. No decorrer do texto o conjunto 
$\gamma(s) \cup \theta_{1} \cup \alpha(s) \cup \theta_{2}$ é denominado ciclo heteroclínico infinito. Observe que aqui usamos $t$ para representar o tempo (variável independente) no plano e $s$ para representar o tempo para o sistema compactificado, o que ficará claro no Capítulo 2.

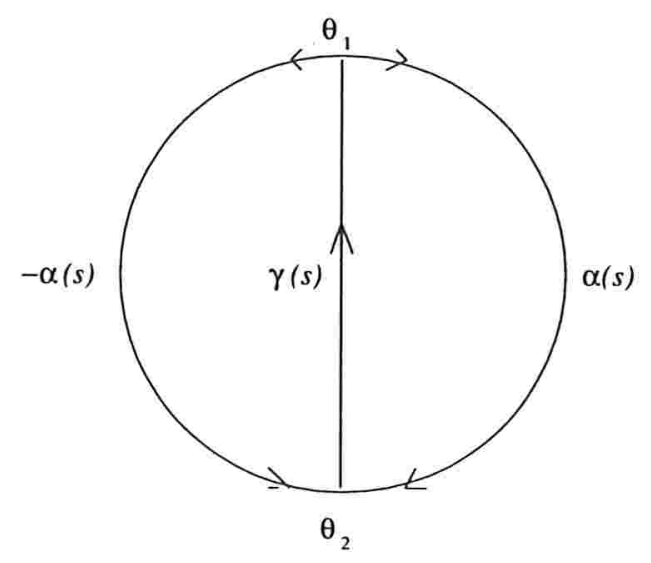

Figura 1.2: Ciclo heteroclínico infinito simétrico de um campo polinomial, no disco de Poincaré.

No Capítulo 3 prova-se (Proposição 3.1.1) que, no caso quadrático ( $n=2$ em (1.5)(1.6)), a forma normal de um sistema satisfazendo as hipóteses acima, considerando-se a reta invariante como sendo $\{x=1\}$, é dada por

$$
\begin{aligned}
& \dot{x}=x y-y \\
& \dot{y}=\alpha+m x+n y+a x^{2}+b x y+c y^{2},
\end{aligned}
$$

$\operatorname{com} \alpha \neq 0,(b+n)^{2}-4(\alpha+m+a) c<0, b^{2}-4 a(c-1)<0$ e $0<c<1$.

Considera-se então a seguinte perturbação periódica do sistema (1.9)-(1.10)

$$
\begin{aligned}
& \dot{x}=x y-y+\mu+A\left(a_{1} \cos \tilde{\omega} t+a_{2} \operatorname{sen} \tilde{\omega} t\right) \\
& \dot{y}=\alpha+m x+n y+a x^{2}+b x y+c y^{2}+A g(\tilde{\omega} t),
\end{aligned}
$$

onde $\mu$ e $A$ são parâmetros reais, $g$ é uma função periódica de período $T=2 \pi / \tilde{\omega}$, sendo $\tilde{\omega}=\omega\left(\sqrt{x^{2}+y^{2}}\right)$, com $\omega>0$ constante. Prova-se (Teorema 3.1.2) que o sistema perturbado (1.11)-(1.12) apresenta duas órbitas periódicas hiperbólicas, $\theta_{1}(s)$ e $\theta_{2}(s)$, 
no infinito, com variedades invariantes locais denotadas por $W_{\mu, A}^{s}\left(\theta_{1}(s)\right)$ e $W_{\mu, A}^{u}\left(\theta_{2}(s)\right)$, e existem funções diferenciáveis $A_{M}(\mu)$ e $A_{m}(\mu)$, com $\mu$ suficientemente pequeno, tais que (ver diagrama de bifurcação no Capítulo 3, Figura (3.2)):

a) se $A=A_{M}(\mu)$ ou $A=A_{m}(\mu)$, então $W_{\mu, A}^{s}\left(\theta_{1}(s)\right)$ e $W_{\mu, A}^{u}\left(\theta_{2}(s)\right)$ se tangenciam quadraticamente;

b) se $\mu>0\left(\mu<0\right.$, respectivamente) e $A_{m}(\mu)<A<A_{M}(\mu)\left(A_{M}(\mu)<A<A_{m}(\mu)\right.$, respectivamente), então $W_{\mu, A}^{s}\left(\theta_{1}(s)\right) \cap W_{\mu, A}^{u}\left(\theta_{2}(s)\right)=\emptyset$;

c) se $A>A_{M}(\mu)$ ou $A<A_{m}(\mu)$, com $\mu>0$, então as variedades invariantes locais $W_{\mu, A}^{s}\left(\theta_{1}(s)\right)$ e $W_{\mu, A}^{u}\left(\theta_{2}(s)\right)$ se intersectam transversalmente em pelo menos um ponto;

d) se $A>A_{m}(\mu)$ ou $A<A_{M}(\mu)$, com $\mu<0$, então vale o mesmo resultado descrito em c).

As curvas $A_{M}(\mu)$ e $A_{m}(\mu)$ obtidas acima (conforme diagrama de bifurcação no Capítulo 3, Figura (3.2)), representam os pontos no espaço de parâmetros para os quais ocorre o "primeiro ponto de tangência" entre as variedades $W_{\mu, A}^{s}\left(\theta_{1}(s)\right)$ e $W_{\mu, A}^{u}\left(\theta_{2}(s)\right)$ das órbitas periódicas no infinito. Considerando as observação feitas na introdução, relacionadas a pontos heteroclinicos transversais, para $\mu$ fixo, suficientemente pequeno, variando-se o parâmetro $A$, desde $A=0$ e na direção vertical observa-se o seguinte cenário (Figura 1.3): se $A<A_{M}(\mu)$, as variedades $W_{\mu, A}^{s}\left(\theta_{1}(s)\right)$ e $W_{\mu, A}^{u}\left(\theta_{2}(s)\right)$ estão separadas (Figura $1.3(\mathrm{a})$ ); se $A=A_{M}(\mu)$, as variedades se tangenciam quadraticamente em um ponto (e, consequentemente, em infinitos pontos) (Fig. 1.3 (b)); se $A>A_{M}(\mu)$, as variedades se intersectam transversalmente em um ponto (e, consequentemente, em infinitos pontos) (Fig. 1.3 (c)). A Figura 1.3 mostra o comportamento das variedades em uma seção transversal ao fluxo do compactificado do sistema perturbado (1.11)-(1.12) (ver Capítulo 3 ), no toro $\mathrm{D}^{2} \times \mathrm{S}^{1}$.

Observe que, embora $W_{\mu, A}^{s}\left(\theta_{1}(s)\right)$ e $W_{\mu, A}^{u}\left(\theta_{2}(s)\right)$ sejam variedades invariantes de órbitas periódicas no infinito, as (infinitas) tangências e intersecções transversais entre elas ocorrem no interior do Disco de Poincaré, ou seja, na parte finita do plano. Trata-se de um fenômeno global, relacionado com as órbitas periódicas no infinito, influenciando 


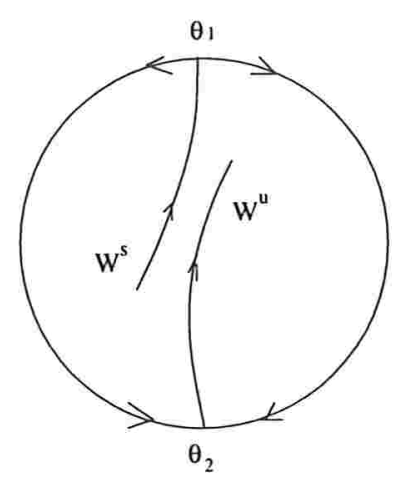

(a)

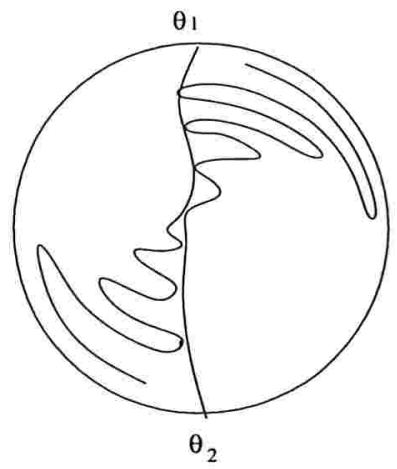

(b)

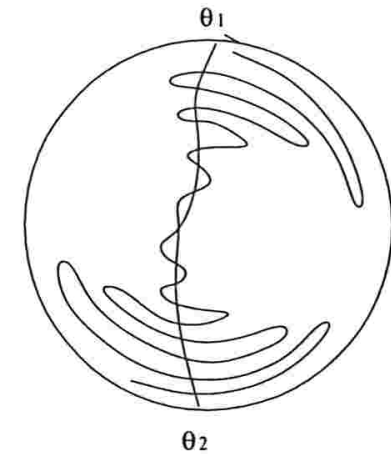

(c)

Figura 1.3: Comportamento das variedade invariantes $W_{\mu, A}^{s}\left(\theta_{1}(s)\right)$ e $W_{\mu, A}^{u}\left(\theta_{2}(s)\right)$ das órbitas periódicas no infinito do sistema perturbado (1.11)-(1.12), em uma seção transversal ao fluxo do sistema compactificado.

a dinâmica das soluções do sistema na parte finita do plano. A esse comportamento complexo das soluções, determinado pelas infintas tangências e intersecções transversais entre as variedades das órbitas periódicas no infinito, chamamos "caos que surge do infinito", na seção anterior.

No caso em que se considera $\tilde{\omega}$ contante no sistema (1.11)-(1.12), em vez de duas órbitas periódicas hiperbólicas, o sistema apresenta duas linhas de singularidades no infinito. Tal caso é estudado através de simulações numéricas, para um exemplo particular, na seção 3.2. Um estudo analítico de tal situação será desenvolvido por nós em um trabalho futuro.

Em seguida, mostra-se que os resultados obtidos com a perturbação periódica da forma $\left(a_{1} \cos \tilde{\omega} t+a_{2} \operatorname{sen} \tilde{\omega} t, g(\tilde{\omega} t)\right)$ acima continuam válidos para uma perturbação mais geral, da forma $(f(\tilde{\omega} t), g(\tilde{\omega} t))$, desde que a função periódica $f$ satisfaça determinada condição de não-degenerescência. As hipóteses são bastante gerais, de modo que se pode concluir que, genericamente, devem ocorrer tais bifurcações. Fica evidente também que o segundo termo, $g(\tilde{\omega} t)$, da função de perturbação, não influencia na quebra das variedades invariantes $W_{\mu, A}^{s}\left(\theta_{1}(s)\right)$ e $W_{\mu, A}^{u}\left(\theta_{2}(s)\right)$, no caso em que se considera a reta 
invariante que conecta os pontos no infinito como sendo vertical.

A análise do caso quadrático é de particular importância, visto que o estudo de vários problemas aplicados, como da Astrofísica (equação de Lane-Endem [3]), interação entre espécies de seres vivos (sistema Lotka-Volterra [14], [18]), e da Mecânica dos Fluidos (equação de Blasius [10]), para os quais é razoável considerar-se perturbações periódicas, se reduz ao estudo de sistemas da forma (1.9)-(1.10) (conforme Capítulo 3). Entretanto, os resultados obtidos para os campos quadráticos são facilmente generalizados para campos polinomiais de grau $n$ com uma reta invariante, usando-se a mesma técnica.

Mudando-se apropriadamente a perturbação e usando-se rotações, ainda no Capítulo 3 , os resultados acima são generalizados para campos polinomiais de grau $n$, com uma curva qualquer invariante, conectando dois pontos de sela hiperbólicos no infinito. Conclui-se também que resultados análogos são válidos para ciclos singulares formados por conexões de um ponto de sela na parte finita do plano com pontos de sela no infintito (aqui denominados de ciclos singulares semi-infinitos).

No Capítulo 4 considera-se o caso em que os ciclos singulares infinitos descritos acima são acumulados por órbitas periódicas, ditas de grande amplitude. Faz-se um estudo das bifurcações apresentadas por tais órbitas em consequência de perturbações periódicas do sistema polinomial. Prova-se a existência de regiões no espaço de parâmetros para as quais o sistema perturbado apresenta órbitas periódica (subharmônicas), que bifurcam das órbitas periódicas de grande amplitude do sistema não-perturbado, que apresentam uma relação de ressonância com o período da função de perturbação.

Faz-se uma análise detalhada do caso em que os polinômios são quadráticos, e o campo possui uma reta invariante e dois centros, cujas órbitas periódicas se acumulam nos ciclos singulares infinitos.

Além disso, estabelecendo-se uma conexão entre as perturbações periódicas dos ciclos heteroclínicos infinitos, consideradas no Capítulo 3, e as bifurcações subharmônicas, prova-se, para o caso quadrático, que, sob certas condições, as tangências heteroclínicas detectadas são limites de bifurcações tipo sela-nó de órbitas subharmônicas. 
Tal resultado estabelece para esta classe de sistemas polinomiais não-hamiltonianos e considerando-se ciclos singulares infinitos, alguns dos resultados obtidos em [8] para a família

$$
\ddot{x}-x+x^{2}=-\lambda_{1} \dot{x}+\lambda_{2} f(t),
$$

com $f(t+1)=f(t)$, sendo $\lambda_{1}$ e $\lambda_{2}$ parâmetros reais.

Em todo o trabalho fica clara a necessidade de uma análise global do retrato de fase dos campos polinomiais, que é desenvolvida via compactificação. 


\section{Capítulo 2}

\section{Construções e Resultados}

\section{Preliminares}

\subsection{Introdução}

Em 1881, Poincaré iniciou o estudo dos campos vetoriais polinomiais do $\mathbb{R}^{2}$ por meio da projeção central de suas soluções na esfera $S^{2}$ [21]. Desta forma, estabeleceu-se as bases para o estudo do comportamento das soluções dos campos polinomiais nas proximidades do infinito que, com este procedimento, passa a ser representado pelo equador $S^{1} \subset S^{2}$. Descritivamente, o método consiste em tomar-se um campo vetorial $X$ do plano e construir, via projeção central, um campo definido no hemisfério aberto superior da esfera e outro no hemisfério aberto inferior e, em seguida, estendê-los ao equador $\mathrm{S}^{1}$, obtendo-se assim um campo analítico definido na esfera toda.

O processo acima é conhecido como compactificação de Poincaré e o campo analítico obtido na esfera é chamado de campo de Poincaré, e denotado por $p(X)$.

No presente trabalho utiliza-se esta técnica no estudo de propriedades de perturbações periódicas dos campos vetoriais polinomiais no plano, envolvendo o infinito. Cabe ressaltar que, no caso de perturbações periódicas, em vez de ser representado pelo 
equador $\mathrm{S}^{1}$, o infinito passa a ser representado por um toro $\mathrm{S}^{1} \times \mathrm{S}^{1}$, conforme veremos.

\subsection{Compactificação usando coordenadas polares}

Considere em $\mathbb{R}^{3}$ a esfera $\mathrm{S}^{2}=\left\{(x, y, z) \in \mathbb{R}^{3} \mid x^{2}+y^{2}+z^{2}=1\right\}$ e sejam $H^{+}=\left\{(x, y, z) \in \mathrm{S}^{2} \mid z>0\right\}$ e $H^{-}=\left\{(x, y, z) \in \mathrm{S}^{2} \mid z<0\right\}$ os hemisférios abertos superior e inferior de $S^{2}$, respectivamente. Seja $\Pi=\left\{(x, y, z) \in \mathbb{R}^{3} \mid z=1\right\}$ o plano tangente a $\mathrm{S}^{2}$ no ponto $(0,0,1)$. Tomando-se uma reta $r$ passando pela origem $(0,0,0)$ e por um ponto $p \in \Pi$, pode-se associar a $p$ dois pontos de $S^{2}, p^{+} \in H^{+}$e $p^{-} \in H^{-}$.

Com isto obtem-se dois difeomorfismos sobrejetivos, $f_{ \pm}: \Pi \longrightarrow H^{ \pm}$, cujas expressões, para coordenadas $(x, y) \in \Pi$ são obtidas tomando-se a equação da reta $r$ e intersectando-a com $\mathrm{S}^{2}$, o que fornece:

$$
f_{+}(x, y)=\frac{(x, y, 1)}{\Delta(x, y)} \quad e \quad f_{-}(x, y)=-\frac{(x, y, 1)}{\Delta(x, y)}
$$

onde $\Delta(x, y)=\sqrt{x^{2}+y^{2}+1}$. Estes difeomorfismos são chamados de projeção central do plano $\Pi$ nos hemisférios superior e inferior de $S^{2}$, respectivamente. Diz-se assim que os pontos do equador $\mathrm{S}^{1} \subset \mathrm{S}^{2}$ correspondem aos pontos no infinito do plano $\Pi$.

Se $X$ é um campo vetorial do $\mathbb{R}^{2}$, usando-se a diferencial da projeção central acima pode-se induzir em $H^{+} \cup H^{-}$o campo $\tilde{X}$ dado por

$$
\tilde{X}(u)=\left\{\begin{array}{c}
D f_{+}(x, y) \cdot X(x, y), \quad \text { se } u=f_{+}(x, y) \in H^{+} \\
D f_{-}(x, y) \cdot X(x, y), \quad \text { se } u=f_{-}(x, y) \in H^{-}
\end{array}\right.
$$

Para estudar o comportamento assintótico das órbitas não-limitadas de $X$, estende-se $\tilde{X}$ continuamente ao equador $\mathrm{S}^{1}=\left\{(x, y, z) \in \mathrm{S}^{2} \mid z=0\right\}$, obtendo-se assim um campo definido na esfera toda. O estudo deste campo estendido, numa vizinhança do equador, fornece informações sobre o comportamento das soluções de $X$ nas proximidades do infinito. No caso de $X$ ser um campo arbitrário do $\mathbb{R}^{2}$ isto nem sempre é possível; entretanto, se $X$ é um campo polinomial tal extensão é possível, conforme a Proposição 
abaixo, que é um resultado clássico da Teoria Qualitativa da Equações Diferenciais Oridinárias.

Proposição 2.2.1 Seja X um campo vetorial polinomial do $\mathbb{R}^{2}$, de grau $n$. Considere a função $\rho: \mathrm{S}^{2} \longrightarrow \mathbb{R}$ dada por $\rho(x, y, z)=z^{n-1}$ e seja $\tilde{X}$ o campo induzido em $\mathrm{S}^{2}-\mathrm{S}^{1}$, definido acima. Então $\rho \tilde{X}$ estende-se unicamente a um campo analítico em $\mathrm{S}^{2}$, que deixa o equador $\mathrm{S}^{1} \subset \mathrm{S}^{2}$ invariante.

Prova: ver [24], [27].

Observação 2.2.2 Multiplicar o campo $\tilde{X}$ por $z^{n-1}$ é equivalente a tomar um novo tempo $s=s(t)$ tal que $\frac{d s}{d t}=\frac{1}{z^{n-1}}$ e considerar a derivaçấo relativamente ao novo tempo $s$, no sistema de equações diferenciais induzido pelo campo $\tilde{X}$. De fato:

$$
\tilde{X}(x)=\frac{d x}{d t}=\frac{d x}{d s} \frac{d s}{d t}=\frac{d x}{d s} \frac{1}{z^{n-1}} .
$$

Mais geralmente, sendo $f: U \rightarrow \mathbb{R}^{\mathbf{n}}$ uma função diferenciável e $g: U \rightarrow \mathbb{R}$ uma função diferenciável positiva, vale o seguinte lema de reparametrização do tempo para soluções de equações diferenciais ordinárias (conforme [5] ou [7])

Lema 2.2.3 Se $J \subset \mathbb{R}$ é um intervalo aberto contendo a origem $e \varphi: J \rightarrow \mathbb{R}^{\mathrm{n}} e^{\prime}$ uma solução da equação diferencial $\dot{x}=f(x)$, com $\varphi(0)=x_{0} \in U$, entẫo a função $B: J \rightarrow \mathbb{R}$ dada por

$$
B(t):=\int_{0}^{t} \frac{1}{g(\varphi(s))} d s
$$

é inversível sobre sua imagem $K \subset \mathbb{R}$. Sendo $\psi: K \rightarrow J$ a inversa de $B$, então a identidade $\psi^{\prime}(t)=g(\varphi(\psi(t)))$ vale para todo $t \in K$, e a função $\sigma: K \rightarrow \mathbb{R}^{\mathrm{n}}$ dada por $\sigma(t)=\varphi(\psi(t))$ é a solução da equação diferencial $\dot{x}=g(x) f(x)$, com condição inicial $\sigma(0)=x_{0}$. 
Prova: A função $s \rightarrow 1 / g(\varphi(s))$ é contínua em $J$. Assim, $B$ está definida em $J$ e sua derivada é positiva em todo ponto. Se $\psi$ é sua inversa, então

$$
\psi^{\prime}(t)=\frac{1}{B^{\prime}(\psi(t))}=g(\varphi(\psi(t)))
$$

e tem-se

$$
\sigma^{\prime}(t)=\psi^{\prime}(t) \varphi^{\prime}(\psi(t))=g(\varphi(\psi(t)) f(\varphi(\psi(t)))=g(\sigma(t)) f(\sigma(t)) .
$$

Assim, pela Proposição 2.2.1, o campo de Poincaré associado a $X$ é dado por $p(X)=$ $\rho \tilde{X}$. Observe a interessante e essencial propriedade do campo $p(X)$ deixar invariante o equador, pois $S^{1}$ representa os pontos do plano $\Pi$ no infinito. As singularidades do campo compactificado $p(X)$ em $\mathrm{S}^{1}$ são chamadas de singularidades no infinito do campo polinomial $X$. Se $p(X)$ não tiver singularidades em $\mathrm{S}^{1}$, então $\mathrm{S}^{1}$ é uma órbita periódica de $p(X)$ e diz-se que o campo polinomial $X$ possui uma órbita periódica no infinito. Pode-se assim, analogamente ao que se faz para singularidades e órbitas periódicas de $X$ em partes compactas de $\mathbb{R}^{2}$, definir o conceito de singularidades e órbitas periódicas hiperbólicas de $X$ no infinito (ver [24], [25], [27]). Define-se também os ciclos singulares infinitos, que são os ciclos formados por uma solução contida em $\mathrm{S}^{2}-\mathrm{S}^{1}$, que conecta dois pontos de sela em $\mathrm{S}^{1}$, unidos por uma solução regular, inteiramente contida em $\mathrm{S}^{1}$ (Figura 1.2, do Capítulo 1).

Como os campos obtidos nos hemisférios superior e inferior da esfera são opostos, diferindo apenas pelo sinal, o que se faz comumente é considerar somente o campo do hemisfério norte, que é homeomorfo ao disco $\mathrm{D}^{2} \subset \mathbb{R}^{2}$, obtendo-se assim um campo definido neste conjunto (conhecido como "Disco de Poincaré" [19]). Assim, os pontos do bordo de $\mathrm{D}^{2}$ correspondem aos pontos no infinito do campo polinomial no plano.

Dado um campo vetorial polinomial $X=(P, Q)$, definido em $\mathbb{R}^{2}$, existem diferentes formas de se obter uma expressão analítica para o campo compactificado $p(X)$, no Disco de Poincaré (ver, por ex., [24], [25] ou [27]). 
Sendo $\mathrm{S}^{2}$ uma variedade diferenciável, escolhendo um sistema de coordenadas conveniente podemos obter a expressão de $p(X)$ nestas coordenadas. Utilizando coordenadas polares $(\theta, \rho), 2 \pi$ periódicas em $\theta$, podemos cobrir o hemisfério superior da esfera menos o polo norte $(0,0,1)$, através da função sobrejetora

$$
F: \mathbb{R} \times[0,+\infty) \longrightarrow \mathrm{H}^{+}-\{(0,0,1)\}
$$

dada por

$$
F(\theta, \rho)=\left(1+\rho^{2}\right)^{-\frac{1}{2}}(\cos \theta, \operatorname{sen} \theta, \rho)
$$

Considere então o campo polinomial

$$
X(x, y)=(P(x, y), Q(x, y))
$$

com

$$
P(x, y)=\sum_{k=0}^{n} P_{k}(x, y) \quad e \quad Q(x, y)=\sum_{k=0}^{n} Q_{k}(x, y),
$$

onde $P_{k}$ e $Q_{k}$ são polinômios homogêneos de grau $k$.

Usando, de um lado a projeção central e de outro a carta $F(\theta, \rho)$ acima, podemos encontrar a expressão do campo compactificado $p(X)$, em coordenadas $(\theta, \rho)$, que é dada por (conforme [25])

$$
p(X)(\theta, \rho)=\left(1+\rho^{2}\right)^{\frac{1-n}{2}}\left(\sum_{i=0}^{n} \rho^{i} A_{n-i}(\theta),-\rho \sum_{i=0}^{n} \rho^{i} R_{n-i}(\theta)\right)
$$

onde

$$
\begin{gathered}
A_{k}(\theta)=-P_{k}(\cos \theta, \operatorname{sen} \theta) \operatorname{sen} \theta+Q_{k}(\cos \theta, \operatorname{sen} \theta) \cos \theta \\
R_{k}(\theta)=P_{k}(\cos \theta, \operatorname{sen} \theta) \cos \theta+Q_{k}(\cos \theta, \operatorname{sen} \theta) \operatorname{sen} \theta
\end{gathered}
$$

para $k=0,1, \ldots, n$, com $\theta \in[0,2 \pi)$ e $\rho \in[0, \infty)$. Assim o campo acima fica definido no conjunto $[0,2 \pi) \times[0,+\infty)$, que pode ser identificado com o disco $D^{2}-\{(0,0)\}$. Da expressão da função $F(\theta, \rho)$ acima segue que, para $\rho=0, \theta$ pertence ao equador da esfera $\mathrm{S}^{2}$, que corresponde aos pontos no infinito do sistema polinomial no plano, que, 
por sua vez, podem ser identificados com os pontos do bordo de $\mathrm{D}^{2}$. Daqui por diante, como referência chamamos o disco $D^{2}-\{(0,0)\}$ acima, onde está definido o campo polinomial compactificado, em coordenadas $(\theta, \rho)$, de disco de Poincaré.

Com isto, é imediato que as singularidades do campo polinomial no infinito são dadas pela equação $A_{n}(\theta)=0$, obtida fazendo-se $\rho=0$ na expressão (2.2). Para verificar a hiperbolicidade destes pontos, usa-se a jacobiana do sistema, que é dada por

$$
\left(\begin{array}{cc}
A_{n}^{\prime}(\theta) & A_{n-1}(\theta) \\
0 & -R_{n}(\theta)
\end{array}\right)
$$

cujos autovalores dependem somente dos termos de grau $n$ e $n-1$ do polinômio.

Como o termo $\left(1+\rho^{2}\right)^{\frac{1-n}{2}}$ que aparece na expressão do campo compactificado (2.2) é estritamente positivo, nos cálculos que serão desenvolvidos na seção seguinte usa-se o campo equivalente

$$
p(X)(\theta, \rho)=\left(\sum_{i=0}^{n} \rho^{i} A_{n-i}(\theta),-\rho \sum_{i=0}^{n} \rho^{i} R_{n-i}(\theta)\right)
$$

obtido de (2.2) dividindo-se a equação por tal termo.

Observação 2.2.4 A expressão (2.6) pode ser também obtida através da mudança de coordenadas (inversão)

$$
x=\frac{\cos \theta}{\rho} \quad y=\frac{\operatorname{sen} \theta}{\rho}
$$

e posterior multiplicação do campo obtido pelo termo $\rho^{n-1}$, para eliminação das singularidades e extensão do campo até $\rho=0$ (que corresponde aos pontos do campo polinomial no infinito). A relação acima, entre $(x, y)$ e $(\theta, \rho)$, também pode ser obtida usando-se a projeçáa central e a carta $F(\theta, \rho)$ definida acima. De fato, os pontos do hemisfério superior da esfera podem ser escritos nas formas

$$
P(x, y)=\frac{1}{\sqrt{x^{2}+y^{2}+1}}(x, y, 1) \quad \text { ou } \quad F(\theta, \rho)=\left(1+\rho^{2}\right)^{-\frac{1}{2}}(\cos \theta, \operatorname{sen} \theta, \rho) .
$$

Igualando-se as coordenadas acima, obtem-se a relação desejada. 
A expressão (2.6) para o múltiplo do campo compactificado é especialmente útil no estudo de propriedades globais do campo $X$, principalmente nas proximidades do infinito, pois é válida no disco todo, exceto na origem $(0,0)$.

\subsection{Compactificação de perturbações periódicas de campos polinomiais planares}

Considere o sistema polinomial de equações diferenciais ordinárias do $\mathbb{R}^{2}$, com perturbação periódica, dado por

$$
\begin{aligned}
& \dot{x}=P(x, y)+\epsilon f(\tilde{\omega} t) \\
& \dot{y}=Q(x, y)+\epsilon g(\tilde{\omega} t),
\end{aligned}
$$

onde $\epsilon$ é um parâmetro real, $P$ e $Q$ são polinômios de grau $n$ em $x$ e $y, f$ e $g$ são funções de classe $C^{k}, k \geq 2$, periódicas de período $T=2 \pi / \tilde{\omega}$, sendo $\tilde{\omega}=\omega\left(\sqrt{x^{2}+y^{2}}\right)^{n-1}$, com $\omega>0$ constante.

Usando a expressão (2.6) da seção anterior, obtem-se a seguinte expressão para a compactificação do sistema acima, em coordenadas $(\theta, \rho)$

$$
\begin{aligned}
& \dot{\theta}=-\operatorname{sen} \theta \sum_{i=0}^{n} \rho^{i} P_{n-i}(\cos \theta, \operatorname{sen} \theta)+\cos \theta \sum_{i=0}^{n} \rho^{i} Q_{n-i}(\cos \theta, \operatorname{sen} \theta)+\epsilon \bar{f}(\theta, \rho \cdot \tilde{\omega} t(s)) \\
& \dot{\rho}=-\cos \theta \sum_{i=0}^{n} \rho^{i+1} P_{n-i}(\cos \theta, \operatorname{sen} \theta)-\operatorname{sen} \theta \sum_{i=0}^{n} \rho^{i+1} Q_{n-i}(\cos \theta \cdot \operatorname{sen} \theta)+\epsilon \bar{g}(\theta, \rho, \tilde{\omega} t(s)),
\end{aligned}
$$

onde

$$
\bar{f}(\theta, \rho, \tilde{\omega} t(s))=\rho^{n}[g(\tilde{\omega} t(s)) \cos \theta-f(\tilde{\omega} t(s)) \operatorname{sen} \theta]
$$

e

$$
\bar{g}(\theta, \rho, \tilde{\omega} t(s))=-\rho^{n+1}[f(\tilde{\omega} s) \cos \theta+g(\tilde{\omega} t(s)) \operatorname{sen} \theta],
$$

sendo $t(s)$ tal que $\frac{d t(s)}{d s}=\rho^{n-1}=1 /\left(\sqrt{x^{2}+y^{2}}\right)^{n-1}$. De forma abreviada. tem-se 


$$
\begin{aligned}
& \dot{\theta}=\sum_{i=0}^{n} \rho^{i} A_{n-i}(\theta)+\epsilon \bar{f}(\theta, \rho, \tilde{\omega} t(s)) \\
& \dot{\rho}=-\sum_{i=0}^{n} \rho^{i+1} R_{n-i}(\theta)+\epsilon \bar{g}(\theta, \rho, \tilde{\omega} t(s)),
\end{aligned}
$$

onde $A_{k}(\theta)$ e $R_{k}(\theta)$ são dados pelas expressões (2.3) e (2.4), respectivamente (seção anterior). Observe que a derivação acima é relativa ao novo tempo $s$, reparametrização do tempo $t$ do sistema no plano (conforme a Observação 2.2.2).

Observação 2.3.1 Daqui em diante será denotado por t o tempo no plano, $\epsilon$ por $s o$ tempo no disco de Poincaré, para o campo compactificado.

Os cálculos acima mostram a influência da perturbação $(f(\tilde{\omega} t), g(\tilde{\omega} t))$ no campo compactificado. Tais funções aparecem nas expressões de $\bar{f}$ e $\bar{g}$, que dependem expliticamente do tempo s. Assim, o campo compactificado, não-autônomo, está definido no toro sólido $\mathrm{D}^{2} \times \mathrm{S}^{1}$, e não mais no disco de Poincaré $\mathrm{D}^{2}$. Para ver isto. basta fazer a mudança de variáveis $\tilde{\omega} t(s)=\phi$, donde tem-se

$$
\dot{\phi}=\frac{d \phi}{d s}=\tilde{\omega} \frac{d t(s)}{d s}=\omega\left(\sqrt{x^{2}+y^{2}}\right)^{n-1} \rho^{n-1}=\omega
$$

já que $\rho^{n-1}=1 /\left(\sqrt{x^{2}+y^{2}}\right)^{n-1}$.

Então, devido ao caráter periódico de $\bar{f}$ e $\bar{g}$, considerando $\phi \in \mathrm{S}^{1}$, obtém-se:

$$
\begin{aligned}
\dot{\theta} & =\sum_{i=0}^{n} \rho^{i} A_{n-i}(\theta)+\epsilon \bar{f}(\theta, \rho, \phi) \\
\dot{\rho} & =-\sum_{i=0}^{n} \rho^{i+1} R_{n-i}(\theta)+\epsilon \bar{g}(\theta, \rho, \phi) \\
\dot{\phi} & =\omega,
\end{aligned}
$$

$\operatorname{com}(\theta, \rho) \in[0,2 \pi) \times[0,+\infty), \phi \in \mathrm{S}^{1}$ e $\omega>0$.

Consequentemente, lembrando que o bordo de $\mathrm{D}^{2}$ é invariante para o sistema não perturbado, os pontos no infinito para o campo (2.7)-(2.8) passam a ser representados pelos pontos do bordo do toro sólido $\mathrm{D}^{2} \times \mathrm{S}^{1}$, dado por $\mathrm{S}^{1} \times \mathrm{S}^{1}$. 
Assim, um ponto de equilíbrio hiperbólico no infinito, $(\bar{\theta}, 0)$, do sistema não perturbado, passa a ser uma órbita periódica $\psi(s)$, para o sistema perturbado, contida em $S^{1} \times S^{1}$ (Figura 2.1), cuja parametrização é dada por

$$
\psi(s)=\left(\bar{\theta}, 0, \omega s+\phi_{0}\right)
$$
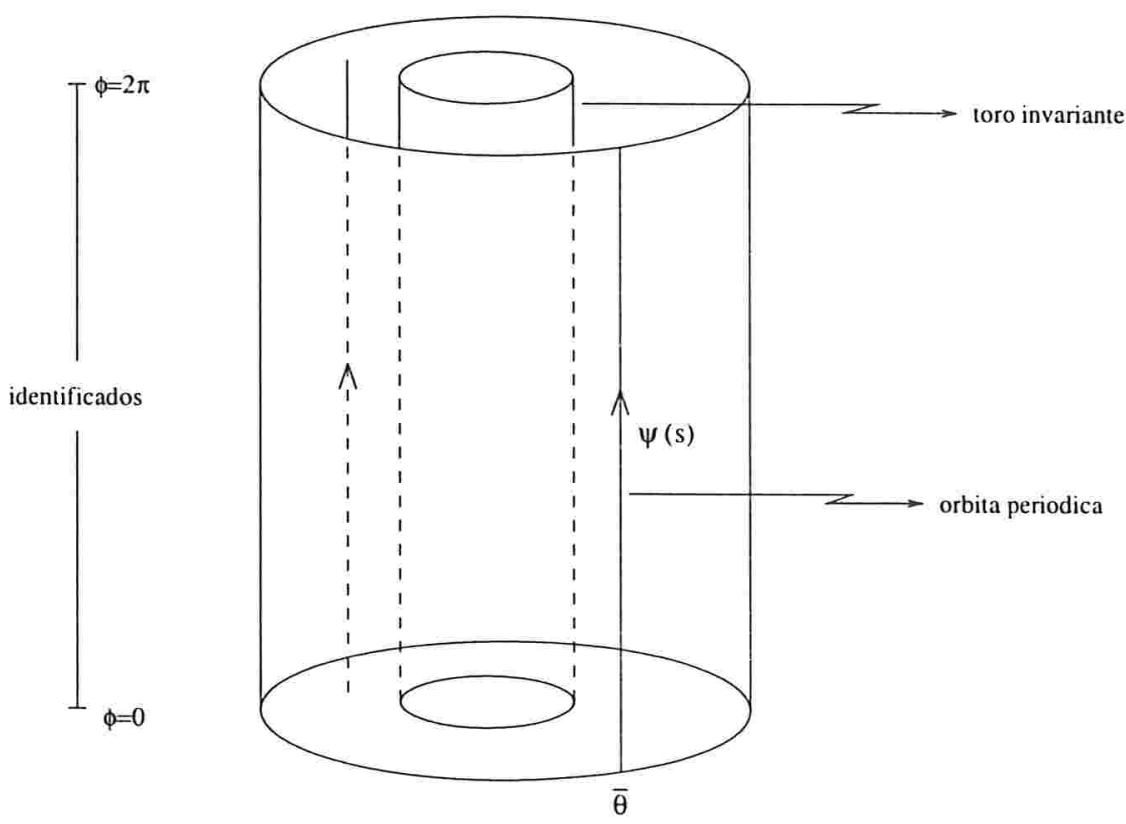

Figura 2.1: Retrato de fase do campo perturbado compactificado, no toro sólido $\mathrm{D}^{2} \times \mathrm{S}^{1}$.

Também, pontos de equilíbrio e órbitas periódicas hiperbólicas do campo nãoperturbado, contidos no interior de $\mathrm{D}^{2}$, passam a ser órbitas periódicas e toros invariantes, respectivamente, do sistema perturbado, contidos no interior do toro sólido $\mathrm{D}^{2} \times \mathrm{S}^{1}$ (Figura 2.1). 


\subsection{A função de separação para variedades invari- antes de órbitas periódicas no infinito}

Suponha que o sistema (2.7)-(2.8) possui, para $\epsilon=0$, dois únicos pontos de equilíbrio simétricos no infinito, que são selas hiperbólicas, isto é, sua compactificação induz um campo de Poincaré $p(X)$ que possui dois pontos de sela simétricos, $\theta_{1}$ e $\theta_{2}$, no bordo do disco $\mathrm{D}^{2}$. Suponha ainda que tais pontos são conectados por uma solução heteroclínica $\gamma(s)$, formando o ciclo singular infinito $\gamma(s) \cup \theta_{1} \cup \alpha(s) \cup \theta_{2}$ (conforme Figura 1.2, mostrada no Capítulo 1, pg.10).

Considerando-se as hipóteses acima e a discussão apresentada na seção anterior, para $\epsilon=0$ o retrato de fase da compactificação de (2.7)-(2.8), no espaço de fase estendido $\mathrm{D}^{2} \times \mathrm{S}^{1}$, tem a forma apresentada na Figura 2.2 .

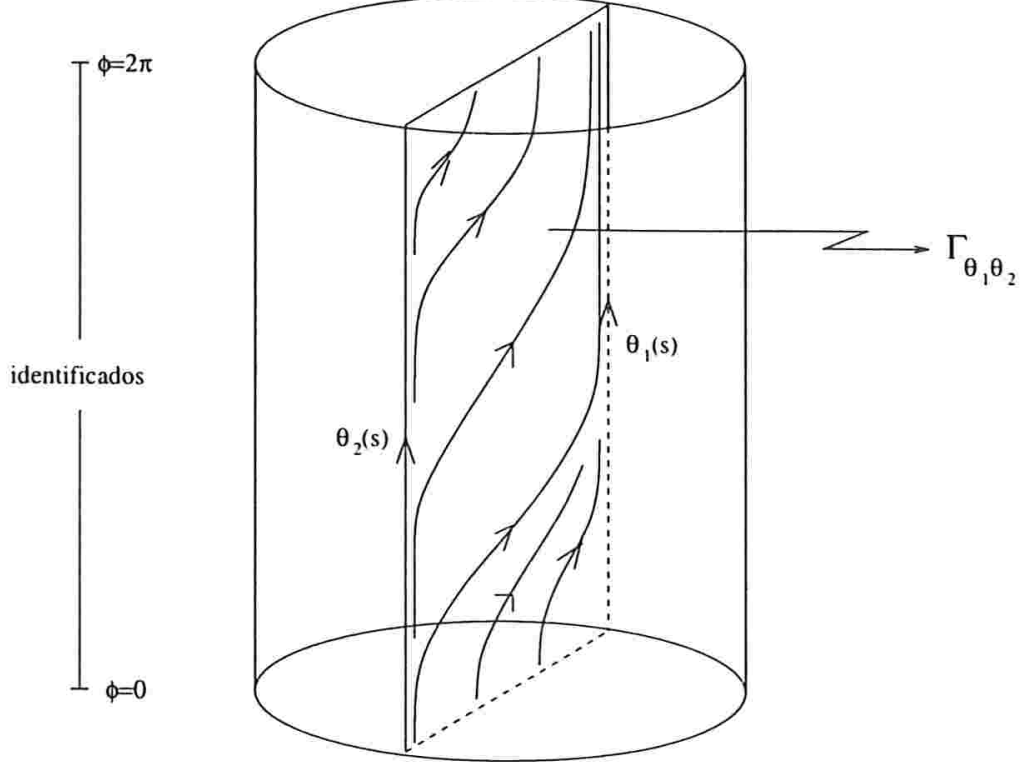

Figura 2.2: Retrato de fase da compactificação de (2.7)-(2.8), para $\epsilon=0$, no plano de fase estendido (toro sólido $\mathrm{D}^{2} \times \mathrm{S}^{1}$ ).

Assim, os pontos de sela $\theta_{1}$ e $\theta_{2}$ do campo de Poincaré induzido por (2.i)-(2.8), 
com $\epsilon=0$, tornam-se, neste espaço de fase estendido, órbitas periódicas hiperbólicas no infinito, $\theta_{1}(s)$ e $\theta_{2}(s)$, e a solução $\gamma(s)$ que conecta $\theta_{1} \operatorname{com} \theta_{2}$ passa a ser uma variedade bidimensional $\Gamma_{\theta_{1} \theta_{2}}$, conectando as órbitas periódicas $\theta_{1}(s)$ e $\theta_{2}(s)$. Logo $\Gamma_{\theta_{1} \theta_{2}}$ é constituída por uma das variedades estáveis de $\theta_{1}(s), W^{s}\left(\theta_{1}(s)\right)$, que coincide com uma das variedades instáveis de $\theta_{2}(s), W^{u}\left(\theta_{2}(s)\right.$ ) (Figura 2.2).

A questão que surge naturalmente neste ponto é a seguinte: como a estrutura esboçada na Figura 2.2 se altera por uma perturbação periódica do campo, isto é, quando considera-se $\epsilon \neq 0$, suficientemente pequeno, em (2.7)-(2.8) (e, consequentemente, no sistema compactificado (2.9)-(2.10))?

Como se trata de duas variedades de dimensão dois, contidas no toro sólido, o que se espera é que, para $\epsilon \neq 0$, as variedades $W^{s}\left(\theta_{1}(s)\right)$ e $W^{u}\left(\theta_{2}(s)\right)$ se tornem transversais, isto é, se separem (intersecção vazia) ou se intersectem ao longo de curvas, conforme mostrado na Figura 2.3.

O restante deste capítulo e o Capítulo 3 inteiro tem por objetivo responder a questão acima. Mais especificamente, estuda-se quais tipos de perturbações deve-se considerar para que se tenha, para $\epsilon \neq 0$ pequeno, intersecção transversal, tangência e separação das variedades invariantes locais de $\theta_{1}(s)$ e $\theta_{2}(s)$, que dependem de $\epsilon$, as quais denotaremos $W_{\epsilon}^{s}\left(\theta_{1}(s)\right)$ e $W_{\epsilon}^{u}\left(\theta_{2}(s)\right)$.

Primeiramente tem-se que, com o procedimento de compactificaçào, o bordo do disco $\mathrm{D}^{2}$ é invariante. Tal propriedade não se altera com a perturbação periódica considerada e, portanto, as porções $\pm \alpha(s) \times S^{1}$ contida no bordo $\mathrm{S}^{1} \times \mathrm{S}^{1}$ do toro sólido (Figura 2.2) não se altera com a perturbação. De fato, fazendo $\rho=0$ no sistema (2.9)-(2.10), tem-se $\dot{\rho}=0$, com o que a solução das equações só apresentam movimento possivelmente nas direções angulares $(\theta, \phi) \in \mathrm{S}^{1} \times \mathrm{S}^{1}$. Também, com a perturbação periódica considerada, que depende somente do tempo, os pontos de sela no infinito não se alteram, pois dependem somente dos termos de grau $n$ do polinômio (lembremos que eles são dados 


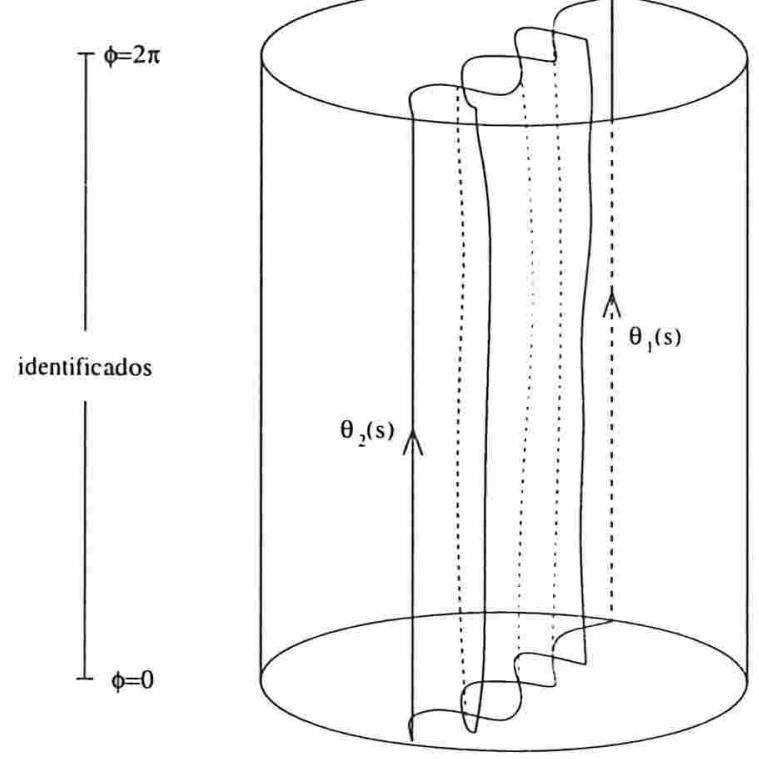

Figura 2.3: Possível intersecção entre as variedades invariantes $\mathbb{W}^{s}\left(\theta_{1}(s)\right)$ e $W^{u}\left(\theta_{2}(s)\right)$ das órbitas periódicas no infinito, em virtude de uma perturbação periódica do sistema (2.7)-(2.8), no plano de fase estendido (toro sólido $\mathrm{D}^{2} \times \mathrm{S}^{1}$ ).

pela solução da equação $\left.A_{n}(\theta)=0\right)$. Consequentemente. as variedades invariantes $W_{\epsilon}^{s}\left(\theta_{1}(s)\right)$ e $W_{\epsilon}^{u}\left(\theta_{2}(s)\right)$ persistesm, para $\epsilon \neq 0$, suficientemente pequeno.

Assim, deve-se analisar apenas o que ocorre com a variedade heteroclínica $\Gamma_{\theta_{1} \theta_{2}}$, em consequência da perturbação periódica.

Para tanto, vamos definir uma função $d(p, \epsilon)$ que dá a distância entre as variedades $W_{\epsilon}^{s}\left(\theta_{1}(s)\right)$ e $W_{\epsilon}^{u}\left(\theta_{2}(s)\right)$, para $\epsilon$ suficientemente pequeno. relativamente a um ponto $p \in \Gamma_{\theta_{1} \theta_{2}}$, em uma seçào transversal a $\Gamma_{\theta_{1} \theta_{2}}$ (para maiores detalhes, ver [29]). Considere a parametrização

$$
\Gamma_{\theta_{1} \theta_{2}}=\left\{(q, \phi) \in D^{2} \times S^{1} \mid q=\gamma\left(s_{0}\right), s_{0} \in \mathbb{R} \text { e } \phi=\phi_{0} \in[0,2 \pi)\right\},
$$

da variedade $\Gamma_{\theta_{1} \theta_{2}}$ e a seção transversal (global) ao fluxo do sistema (2.9)-(2.10), dada 
por (Figura 2.4):

$$
\Sigma^{\phi_{0}}=\left\{(\theta, \rho, \phi) \mid(\theta, \rho) \in[0,2 \pi) \times[0,+\infty) \text { e } \phi=\phi_{0} \in[0.2 \pi)\right\} .
$$

Tome um ponto $p=p\left(s_{0}, \phi_{0}\right) \in \Gamma_{\theta_{1} \theta_{2}} \cap \Sigma^{\phi_{0}}$ e o vetor $\pi_{p}=\left(X^{\perp}\left(\neg\left(s_{0}\right)\right), 0\right)$, onde $X^{\perp}$ representa o campo ortogonal a (2.9)-(2.10), $\operatorname{com} \epsilon=0$ (Figura 2.4).
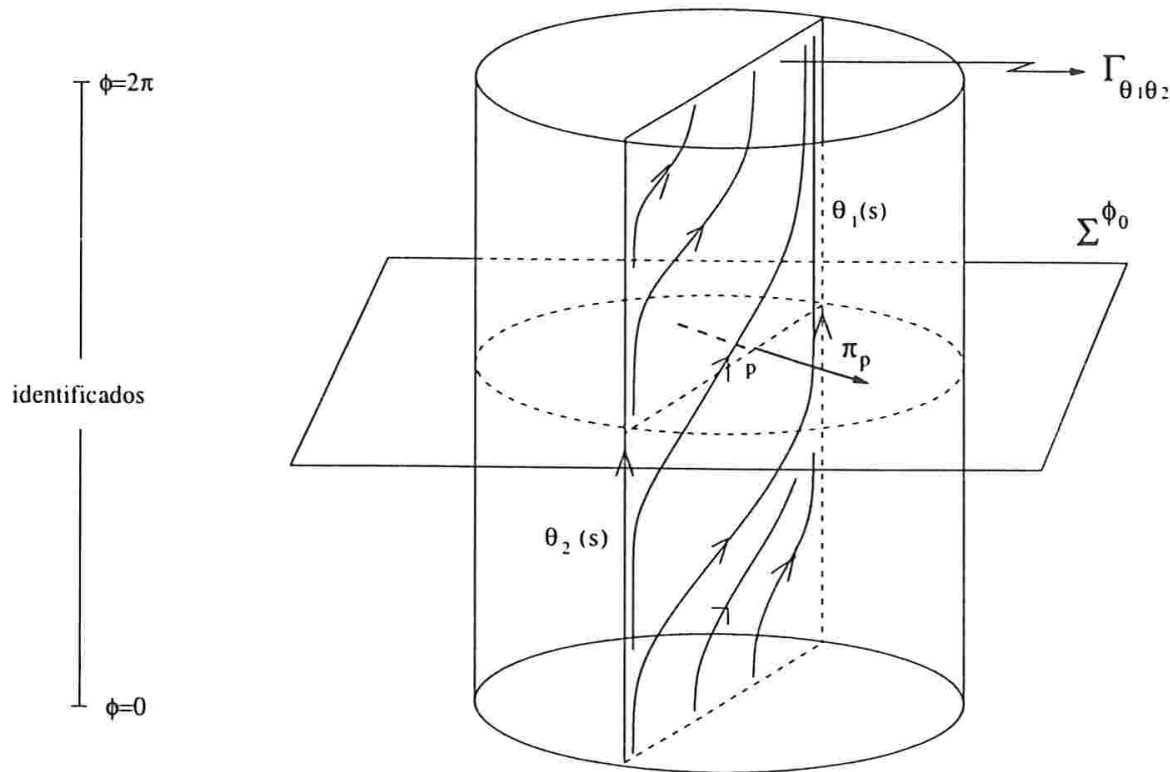

Figura 2.4: Seção $\Sigma^{\phi_{0}}$, transversal ao fluxo do sistema (2.9)-(2.10), com $\epsilon=0$. e vetor $\pi_{p}$ ao longo do qual será medida a distáncia entre as variedades $W_{\epsilon}^{s}\left(\theta_{1}(s)\right)$ e $W_{\epsilon}^{u}\left(\theta_{2}(s)\right)$, para $\epsilon \neq 0$ suficientemente pequeno.

Com a construção acima, $\pi_{p}$ é transversal a $\Gamma_{\theta_{1} \theta_{2}}$ em cada ponto $p$. donde, para $\epsilon=0, W_{0}^{s}\left(\theta_{1}(s)\right)$ e $W_{0}^{u}\left(\theta_{2}(s)\right)$ intercectam a reta gerada por $\pi_{p}$. Entào, pelo Teorema das Variedades Invariantes, juntamente com o Teorema da Dependência Contínua das soluções com relação a parâmetros, para $\epsilon \neq 0$ suficientemente pequeno. $W_{\epsilon}^{s}\left(\theta_{1}(s)\right)$ e $W_{\epsilon}^{u}\left(\theta_{2}(s)\right)$, também intersectam a reta gerada por $\pi_{p}$ em pontos $q_{\epsilon}^{s}\left(s_{0}\right)$ e $q_{\epsilon}^{u}\left(s_{0}\right)$, respectivamente (Figura 2.5).

Define-se então a função de separaçào entre as variedades invariantes locais $W_{\epsilon}^{s}\left(\theta_{1}(s)\right)$ 


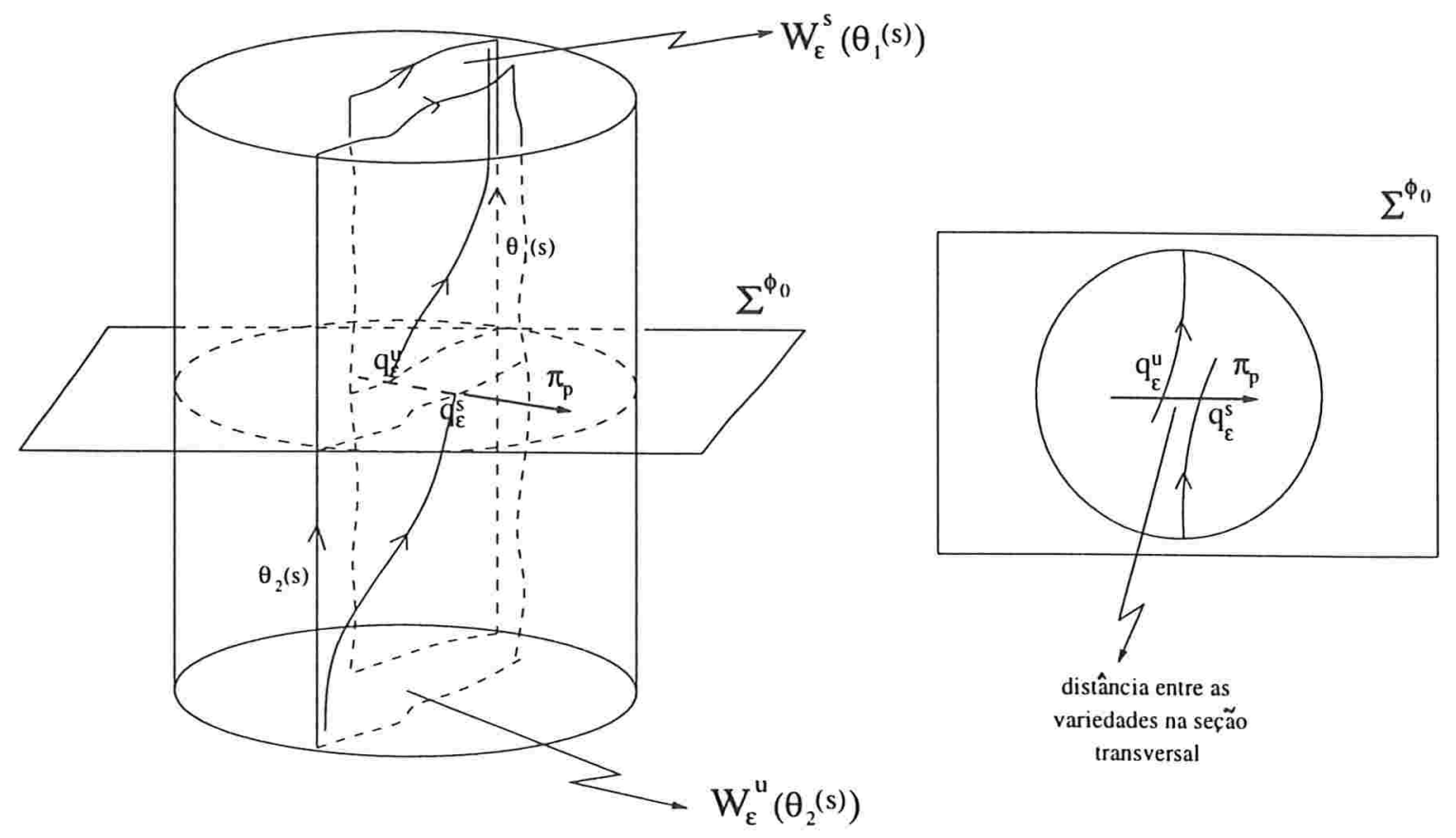

Figura 2.5: Distancia entre as variedades invariantes em uma seção transversal $\Sigma^{\phi_{0}}$.

e $W_{\epsilon}^{u}\left(\theta_{2}(s)\right)$, relativamente ao ponto $p=p\left(s_{0}, \phi_{0}\right) \in \Gamma_{\theta_{1} \theta_{2}} \cap \Sigma^{\phi_{0}}$ (Figura 2.5) como sendo

$$
d\left(s_{0}, \phi_{0}, \epsilon\right)=\frac{<\left(q_{\epsilon}^{u}\left(s_{0}\right)-q_{\epsilon}^{s}\left(s_{0}\right)\right), X^{\perp}\left(\gamma\left(s_{0}\right)\right)>}{\left\|X^{\perp}\left(\gamma\left(s_{0}\right)\right)\right\|},
$$

onde $q_{\epsilon}^{u}\left(s_{0}\right)$ e $q_{\epsilon}^{s}\left(s_{0}\right)$ sào pontos iniciais das soluções $q_{\epsilon}^{u}\left(s+s_{0}\right)$ e $q_{\epsilon}^{s}\left(s+s_{0}\right)$ do sistema perturbado (2.9)-(2.10). contidas em $W_{\epsilon}^{u}\left(\theta_{2}(s)\right)$ e $W_{\epsilon}^{s}\left(\theta_{1}(s)\right)$, respectivamente, que cortam a reta gerada por $\pi_{p}$ pela primeira vez antes de tenderem a $\theta_{2}(s)$ ("no passado") e a $\theta_{1}(s)$ ("no futuro"), respectivamente (para maiores detalhes, ver [29], Capítulo 4).

Observe que, como o vetor $\left(q_{\epsilon}^{u}\left(s_{0}\right)-q_{\epsilon}^{s}\left(s_{0}\right)\right)$ é paralelo ao vetor $\pi_{p}$, então a norma da função de separação definida acima dá exatamente a norma do vetor $\left(q_{\epsilon}^{u}\left(s_{0}\right)-q_{\epsilon}^{s}\left(s_{0}\right)\right)$. É claro também, da definição, que $d\left(s_{0}, \phi_{0}, \epsilon\right)=0$ se, e somente se, $q_{\epsilon}^{u}\left(s_{0}\right)=q_{\epsilon}^{s}\left(s_{0}\right)$.

Para $\epsilon=0$, tem-se $d\left(s_{0}, \phi_{0}, 0\right)=0$. Então, a expansão em Série de Taylor, com 
relação a $\epsilon$, em torno de $\epsilon=0$, da função de separação definida acima, é dada por

$$
d\left(s_{0}, O_{0} . \epsilon\right)=\epsilon \frac{\partial d\left(s_{0}, \phi_{0} .0\right)}{\partial \epsilon}+O\left(\epsilon^{2}\right)
$$

O primeiro termo nào identicamente nulo na expansào acima é dado por

$$
\frac{\partial d\left(s_{0} \cdot \phi_{0}, 0\right)}{\partial \epsilon}=\frac{M\left(s_{0}, \phi_{0}\right)}{\left\|X^{\perp}\left(\gamma\left(s_{0}\right)\right)\right\|} .
$$

A função $M\left(s_{0}, \phi_{0}\right)$ acima desempanha um papel fundamental no estudo da separação entre as variedades e possui uma representação integral dada por (conforme [5], pg.396 e seguintes, ou [1.5])

$$
M\left(s_{0}, \phi_{0}\right)=\int_{-\infty}^{+\infty} e^{-\int_{0}^{s} \operatorname{div} X\left(\gamma\left(\tau+s_{0}\right)\right) d \tau}<X^{\perp}\left(\gamma\left(s+s_{0}\right)\right) \cdot F\left(\gamma\left(s+s_{0}\right), \omega s+\phi_{0}\right)>d s,
$$

onde $X=\left(X^{1}, X^{2}\right)$ é o sistema compactificado dado em $(2.9)-(2.10)$, com $\epsilon=0$, $X^{\perp}=\left(-X^{2}, X^{1}\right), F=(\bar{f}, \bar{g})$ são as coordenadas da função de perturbação no sistema compactificado, e $\gamma(s)$ é a trajetória heteroclínica que conecta os equilíbrios $\theta_{1} \operatorname{com} \theta_{2}$, no disco de Poincaré.

A fórmula integral (2.11) para a derivada parcial da função de separação com relação ao parâmetro é, provavelmente. conhecida desde Poincaré. Também foi descoberta independentemente por vários autores, dentre os quais citamos Sotomayor, em [23], ao estudar perturbações autônomas de conexões de pontos de sela no plano, e Melnikov, em [20], no estudo de perturbações periódicas de campos vetoriais no plano, contendo órbitas homoclínicas e heteroclínicas. Neste trabalho, a fórmula integral (2.11) para $M\left(s_{0}, \phi_{0}\right)$ será chamada Integral de Melnikov-Sotomayor.

É importante notar que a expressão integral depende apenas do sistema não-perturbado e da função de perturbação $F=(\bar{f}, \bar{g})$, calculados na órbita heteroclínica $\gamma(s)$.

No caso em que o sistema é hamiltoniano, o divergente se anula e a função $M$ toma a forma

$$
M\left(s_{0}, \phi_{0}\right)=\int_{-\infty}^{+\infty}<X^{\perp}\left(\gamma\left(s+s_{0}\right)\right), F\left(\gamma\left(s+s_{0}\right), \omega s+\phi_{0}\right)>d s .
$$


Observação 2.4.1 Da definiçào da distância d $\left(s_{0}, \phi_{0}, \epsilon\right)$ pode-se observar que a função acima é obtida resolvendo-se a equação variacional associada a (2.9)-(2.10), derivando a equação com relação ao parâmetro $\epsilon$. Assim. a função $M\left(s_{0}, \phi_{0}\right)$ é dada pela projeção da solução da equação variacional na direção ortogonal. Para uma referência sobre a dedução da função .M, ver, além dos trabalhos originais [20] e [23], os livros [29], Capítulo 4, e [5], Capítulo 6, onde é apresentada detalhadamente, de forma didática, a dedução da fórmula integral acima.

Vale então o seguinte teorema clássico da Teoria das Perturbações, cuja demonstração, que aqui omitiremos, aparece em vários textos e artigos ([5],[15],[29]), sendo que, pelo que temos conhecimento, foi publicada pela primeira vez em [20].

Teorema 2.4.2 Suponha que existe um ponto $\left(\bar{s}_{0}, \bar{\phi}_{0}\right) \in \mathbb{R} \times \mathrm{S}^{1}$ tal que

$$
M\left(\overline{s_{0}}, \overline{\phi_{0}}\right)=0 \quad e \quad \frac{\partial M}{\partial s_{0}}\left(\overline{s_{0}}, \overline{\phi_{0}}\right) \neq 0 .
$$

Então, para $\epsilon \neq 0$ suficientemente pequeno, as variedades invariantes $W_{\epsilon}^{s}\left(\theta_{1}(s)\right)$ e $W_{\epsilon}^{u}\left(\theta_{2}(s)\right)$ do sistema (2.9)-(2.10) se intersectam transversalmente no ponto $\left(\gamma\left(\overline{s_{0}}\right)+\right.$ $\left.O(\epsilon), \bar{\phi}_{0}\right)$. Além disso, se $M\left(s_{0}, \phi_{0}\right) \neq 0$ para todo ponto $\left(s_{0}, \phi_{0}\right) \in \mathbb{R} \times \mathrm{S}^{1}$, então $W_{\epsilon}^{s}\left(\theta_{1}(s)\right) \cap W_{\epsilon}^{u}\left(\theta_{2}(s)\right)=\emptyset$.

Prova: Trata-se de uma aplicação do Teorema da Funçào Implícita à função de separação definida acima. Ver, por exemplo, [5], Capítulo 6, onde a demonstração é feita com todos os detalhes.

O seguinte Lema será útil nas seções seguintes.

Lema 2.4.3 Para a função de Melnikov-Sotomayor $M\left(s_{0}, \phi_{0}\right)$, definida em (2.11). tem-se que, se $M\left(\overline{s_{0}}, \overline{\phi_{0}}\right)=0$, então $\frac{\partial M}{\partial s_{0}}\left(\overline{s_{0}}, \overline{\phi_{0}}\right) \neq 0$ se, e somente se, $\frac{\partial M}{\partial \phi_{0}}\left(\overline{s_{0}}, \bar{\phi}_{0}\right) \neq 0$. 
Prova: Fazendo a mudança de variáveis $\xi=s+s_{0}$ na integral.$M\left(s_{0} . o_{0}\right)$ obtem-se

$$
M\left(s_{0}, \phi_{0}\right)=\int_{-\infty}^{+\infty} e^{-\int_{0}^{\xi-s_{0}} \operatorname{div} X\left(\gamma\left(\tau+s_{0}\right)\right) d \tau}<X^{\perp}(\gamma(\xi)), F\left(\gamma(\xi) \cdot \omega \xi-\omega s_{0}+\phi_{0}\right)>d \xi .
$$

Fazendo agora a mudança $u=\tau+s_{0}$ na integral do argumento da exponencial que aparece no integrando da função acima, tem-se

$$
M\left(s_{0}, \phi_{0}\right)=\int_{-\infty}^{+\infty} e^{-\int_{s_{0}}^{\xi} \operatorname{div} X(\gamma(u)) d u}<X^{\perp}(\gamma(\xi)), F\left(\gamma(\xi), \omega \xi-\omega s_{0}+\phi_{0}\right)>d \xi .
$$

Derivando a expressão acima com relação a $s_{0}$ e com relação a $\phi_{0}$, chega-se a seguinte relação

$$
\frac{\partial M}{\partial s_{0}}\left(s_{0}, \phi_{0}\right)=\operatorname{div} \cdot X\left(\gamma\left(s_{0}\right)\right) M\left(s_{0}, \phi_{0}\right)+\frac{1}{\omega} \frac{\partial M}{\partial \phi_{0}}\left(s_{0}, \phi_{0}\right) .
$$

Portanto, aplicando as derivadas acima no ponto $\left(\overline{s_{0}}, \overline{\phi_{0}}\right)$ e considerando-se a hipótese $M\left(\overline{s_{0}}, \overline{\phi_{0}}\right)=0$ segue o resultado.

Do lema acima segue que o Teorema 2.4 .2 continua válido se trocarmos, na sua hipótese, a condição $\frac{\partial M}{\partial s_{0}}\left(\overline{s_{0}}, \overline{\phi_{0}}\right) \neq 0$ por $\frac{\partial M}{\partial \phi_{0}}\left(\overline{s_{0}}, \bar{\phi}_{0}\right) \neq 0$. Isto traz algumas vantagens com relação aos cálculos, como ficará claro nas próximas seções.

Se a perturbação $F=(\bar{f}, \bar{g})$ de (2.9)-(2.10) for uma função que depende diferenciavelmente de um parâmetro $\nu$, a função de Melnikov-Sotomayor definida acima também dependerá diferenciavelmente deste parâmetro e vale o seguinte resultado (conforme [15], Teorema 4.5.4, pg.190)

Teorema 2.4.4 Suponha que existe um ponto $\left(\overline{s_{0}}, \bar{\phi}_{0}, \bar{\nu}\right) \in \mathbb{R} \times \mathrm{S}^{1} \times \mathbb{R}$ tal que

$$
\begin{aligned}
\text { i) } M\left(\overline{s_{0}}, \overline{\phi_{0}}, \bar{\nu}\right) & =0, \\
\text { ii) } \frac{\partial M\left(\overline{s_{0}}, \bar{\phi}_{0}, \bar{\nu}\right)}{\partial \phi_{0}} & =0, \\
\text { iii) } \frac{\partial^{2} M\left(\overline{s_{0}}, \bar{\phi}_{0}, \bar{\nu}\right)}{\partial \phi_{0}{ }^{2}} & \neq 0,
\end{aligned}
$$




$$
\text { iv) } \frac{\partial M\left(\overline{s_{0}}, \overline{\phi_{0}} \cdot \bar{\nu}\right)}{\partial \nu} \neq 0
$$

Então, para $\epsilon \neq 0$ suficientemente pequeno, $W_{\epsilon}^{s}\left(\theta_{1}(s)\right)$ e $W_{\epsilon}^{u}\left(\theta_{2}(s)\right)$ se tangenciam quadraticamente no ponto $\left(\hat{\imath}\left(\overline{s_{0}}\right)+O(\epsilon), \bar{\phi}_{0}\right)$, e tal tangência persiste para $\nu=\bar{\nu}+O(\epsilon)$.

Prova: Ver [1.5], pg.190.

\subsection{A integral de Melnikov-Sotomayor em coorde- nadas polares}

Supondo-se, sem perda de generalidade, que a reta invariante do sistema polinomial no plano não passa pela origem, tem-se, consequentemente, que a trajetória heteroclínica $\gamma(s)$, do sistema compactificado. não passa pela origem do disco $\mathrm{D}^{2}$. Pode-se então usar a expressão da compactificação do sistema polinomial

$$
\begin{aligned}
& \dot{x}=P(x, y)+\epsilon f(\tilde{\omega} t) \\
& \dot{y}=Q(x, y)+\epsilon g(\tilde{\omega} t),
\end{aligned}
$$

em coordenadas $(\theta, \rho)$, dada por

$$
\begin{aligned}
& \dot{\theta}=-\operatorname{sen} \theta \sum_{i=1}^{n} \rho^{i} P_{n-i}(\cos \theta \cdot \operatorname{sen} \theta)+\cos \theta \sum_{i=0}^{n} \rho^{i} Q_{n-i}(\cos \theta, \operatorname{sen} \theta)+\epsilon \bar{f}(\rho, \theta, \phi) \\
& \dot{\rho}=-\cos \theta \sum_{i=0}^{n} \rho^{i+1} P_{n-i}(\cos \theta \cdot \operatorname{sen} \theta)-\operatorname{sen} \theta \sum_{i=0}^{n} \rho^{i+1} Q_{n-i}(\cos \theta \cdot \operatorname{sen} \theta)+\epsilon \bar{g}(\rho, \theta, \phi) \\
& \dot{\phi}=\omega,
\end{aligned}
$$

onde

$$
\bar{f}(\rho, \theta, o)=\rho^{n}(g(\phi) \cos \theta-f(\phi) \operatorname{sen} \theta)
$$




$$
\bar{g}(\rho, \theta, \phi)=-\rho^{n+1}(f(\phi) \cos \theta+g(\phi) \operatorname{sen} \theta),
$$

para o cálculo da função integral de Melnikov-Sotomayor definida na seção anterior.

Façamos os cálculos dos termos que entram no integrando da função $M\left(s_{0}, \phi_{0}\right)$. Omitindo, por um momento, os argumentos das funçòes, e chamando $s=\operatorname{sen} \theta$ e $c=\cos \theta$. obtemos:

$$
\begin{gathered}
<X^{\perp},(\bar{f}, \bar{g})>= \\
<\left(c \sum_{i=0}^{n} \rho^{i+1} P_{n-i}+s \sum_{i=0}^{n} \rho^{i+1} Q_{n-i},-s \sum_{i=0}^{n} \rho^{i} P_{n-i}+c \sum_{i=0}^{n} \rho^{i} Q_{n-i}\right),(\bar{f}, \bar{g})>= \\
c \bar{f} \sum_{i=0}^{n} \rho^{i+1} P_{n-i}+s \bar{f} \sum_{i=0}^{n} \rho^{i+1} Q_{n-i}-s \bar{g} \sum_{i=0}^{n} \rho^{i} P_{n-i}+c \bar{g} \sum_{i=0}^{n} \rho^{i} Q_{n-i} .
\end{gathered}
$$

Substituindo as expressões de $\bar{f}$ e $\bar{g}$ acima, obtem-se, após alguns cálculos simples, envolvendo propriedades das funções trigonométricas, a igualdade

$$
\begin{aligned}
<X^{\perp},(\bar{f}, \bar{g})> & =g \rho^{n+1} \sum_{i=0}^{n} \rho^{i} P_{n-i}-f \rho^{n+1} \sum_{i=0}^{n} \rho^{i} Q_{n-i} \\
& =\rho^{n+1}<\left(-\sum_{i=0}^{n} \rho^{i} Q_{n-i}, \sum_{i=0}^{n} \rho^{i} P_{n-i}\right),(f, g)>,
\end{aligned}
$$

onde $P_{k}$ e $Q_{k}$ são polinômios homogêneos de grau $k$, componentes dos polinômios $P$ e $Q$ que determinam o campo não perturbado no plano, isto é, $P=\sum_{k=0}^{n} P_{k}$ e $Q=\sum_{k=0}^{n} Q_{k}$, $f$ e $g$ são as funções de perturbação do campo polinomial $(P, Q)$ no plano, e $<,>$ denota o produto interno usual do $\mathbb{R}^{2}$.

O outro termo que entra na Integral de Melnikov-Sotomayor é o divergente do campo compactificado não-perturbado, que é dado por

$$
\sigma(s)=\sum_{i=0}^{n} \rho(s)^{i} A_{n-i}^{\prime}(\theta(s))-\sum_{i=0}^{n}(i+1) \rho(s)^{i} R_{n-i}(\theta(s)) .
$$

Em resumo, a integral (2.11) para o campo compactificado, em coordenadas $(\theta, \rho)$ é dada por

$$
M\left(s_{0}, \phi_{0}\right)=\int_{-\infty}^{+\infty} e^{-\int_{0}^{s} \sigma\left(\tau+s_{0}\right) d \tau} \rho\left(s+s_{0}\right)^{n+1} F\left(\theta, \rho, s, s_{0}, \phi_{0}\right) d s,
$$

onde

$$
\sigma(s)=\sum_{i=0}^{n} \rho(s)^{i} A_{n-i}^{\prime}(\theta(s))-\sum_{i=0}^{n}(i+1) \rho(s)^{i} R_{n-i}(\theta(s)),
$$




$$
\begin{array}{r}
F\left(\theta, \rho, s, s_{0}, \phi_{0}\right)=-f\left(\omega s+o_{0}\right) \sum_{i=0}^{n} \rho\left(s+s_{0}\right)^{i} Q_{n-i}\left(\cos \theta\left(s+s_{0}\right), \operatorname{sen} \theta\left(s+s_{0}\right)\right)+ \\
g\left(\omega s+\phi_{0}\right) \sum_{i=0}^{n} \rho\left(s+s_{0}\right)^{i} P_{n-i}\left(\cos \theta\left(s+s_{0}\right), \operatorname{sen} \theta\left(s+s_{0}\right)\right),
\end{array}
$$

sendo $\gamma(s)=(\theta(s), \rho(s))$ a expressão da conexão heteroclínica entre os equilíbrios $\theta_{1}$ e $\theta_{2}$, em coordenadas $(\theta, \rho)$, no disco de Poincaré, e $\omega s+\phi_{0}$ é a solução de $\dot{\phi}=\omega$ tal que $\phi(0)=\phi_{0}$.

A solução $\gamma(s)=(\theta(s), \rho(s))$ é uma reparametrizacação da solução não-limitada $\varphi(t)=(x(t), y(t))$ do sistema polinomial (2.7)-(2.8) no plano, com $\epsilon=0$, contida na reta invariante $\{x=1\}$, que conecta os pontos de sela hiperbólicos no infinito. Conforme [7], devido a hiperbolicidade dos pontos no infinito, tal solução está definida no intervalo $\left(t_{-}, t_{+}\right)$, com $t_{-}$e $t_{+}$finitos (ver também a Proposição (3.1.1) adiante, onde são feitos os cálculos explícitos da solução, no caso quadrático). Então, com base no Lema 2.2.3 e observação 2.2 .2 , fazendo a mudança de variáveis

$$
t=t(s) \quad \operatorname{com} \quad \frac{d t}{d s}=\rho^{n-1}
$$

na integral (2.14) e usando as relações entre as coordenadas $(\theta, \rho)$ e $(x, y)$, dadas por (conforme observação 2.2.4)

$$
\cos \theta=\rho x . \quad \operatorname{sen} \theta=\rho y \quad e \quad \rho=\frac{1}{\sqrt{x^{2}+y^{2}}} .
$$

obtem-se a integral de Melnikov-Sotomayor (2.14) do campo compactificado, em coordenadas $(x, y)$ do plano, que é dada por

$$
M\left(t_{0}, \phi_{0}\right)=\int_{t_{-}}^{t_{+}} e^{\left.-\int_{0}^{t} \tilde{\sigma}\left(\tau+t_{0}\right)\right) d \tau}\left(x\left(t+t_{0}\right)^{2}+y\left(t+t_{0}\right)^{2}\right)^{-\frac{n+2}{2}} G\left(x, y, t_{0}, \phi_{0}\right) d t
$$

onde

$$
G\left(x, y, t_{0}, \phi_{0}\right)=g\left(\omega t+\phi_{0}\right) P\left(x\left(t+t_{0}\right), y\left(t+t_{0}\right)\right)-f\left(\omega t+\phi_{0}\right) Q\left(x\left(t+t_{0}\right), y\left(t+t_{0}\right)\right),
$$

$\tilde{\sigma}(t)$ é um polinômio de grau $n+1$ em $x(t)$ e $y(t)$, multiplicado por $\|(x(t), y(t))\|^{-(n+1)}$, obtido do divergente $\sigma(s)$ do campo compactificado (expressão 2.13), através das re- 
laçòes (2.15), $t_{0} \in\left(t_{-}, t_{+}\right)$é o correspondente de $s_{0} \in(-\infty,+\infty)$ (conforme o Lema 2.2.3), $P(x, y)$ e $Q(x . y)$ são os polinômios que definem o campo polinomial nãoperturbado,

$$
\begin{aligned}
& \dot{x}=P(x, y) \\
& \dot{y}=Q(x, y)
\end{aligned}
$$

no plano.

As expressões (2.14) e (2.16) serão usadas nos próximos capítulos no estudo de perturbações periódicas de ciclos singulares infinitos e de bifurcações subharmônicas de órbitas periódicas de grande amplitude de campos polinomiais no plano. 


\section{Capítulo 3}

\section{Perturbações Periódicas de Ciclos Heteroclínicos Infinitos}

\subsection{Caso 1: Campos quadráticos com uma reta in- variante}

Considere em $\mathbb{R}^{2}$ um campo vetorial polinomial quadrático que deixa invariante uma reta $r$, sobre a qual não existem pontos críticos do campo. Supondo, sem perda de generalidade, que tal reta é dada pelo eixo- $y$, o sistema de equações diferenciais associado ao campo tem a forma

$$
\begin{aligned}
& \dot{x}=x(\beta+d x+e y) \\
& \dot{y}=\alpha+m x+n y+a x^{2}+b x y+c y^{2} .
\end{aligned}
$$

Considerando agora uma mudança de coordenadas afim, após renomear as constantes e variáveis, o sistema toma a forma

$$
\begin{aligned}
& \dot{x}=x y \\
& \dot{y}=\alpha+m x+n y+a x^{2}+b x y+c y^{2},
\end{aligned}
$$


$\operatorname{com} n^{2}-4 \alpha c<0$.

Campos vetoriais da forma acima aparecem no estudo de muitos problemas aplicados [10]. Por exemplo, sistemas do tipo Lotka-Volterra [14], [18], dados por

$$
\begin{aligned}
& \dot{x}=x(a x+b y+c) \\
& \dot{y}=y(d x+e y+f),
\end{aligned}
$$

com $a, b, c, d, e, f \in \mathbb{R}$, utilizados no estudo da dinâmica populacional de espécies que interagem entre si, podem ser transformados em um sistema do tipo (3.1)-(3.2), com uma mudança afim de coordenadas.

Também, a equaçào de Lane-Endem, de índice $n$, relacionada a problemas da Astrofísica [3], dada por

$$
\left(\xi^{2} \eta^{\prime}\right)^{\prime}+\xi^{\lambda} \eta^{n}=0
$$

onde $\eta^{\prime}=d \eta / d \xi$. é transformada pela mudança de variáveis

$$
x=\frac{\xi \eta^{\prime}}{\eta}, \quad y=\frac{\xi^{\lambda-1} \eta^{n}}{\eta^{\prime}}, \quad t=\ln |\xi|
$$

no sistema

$$
\begin{aligned}
& \dot{x}=-x(1+x+y) \\
& \dot{y}=y(\lambda+1+n x+y),
\end{aligned}
$$

que, por uma mudança afim de coordenadas e multiplicação por um número positivo. se reduz a um sistema da forma (3.1)-(3.2).

Similarmente. a equaçào de Blasius da Mecânica dos Fluidos [10], $\eta^{\prime \prime \prime}+\eta \eta^{\prime \prime}=0$ é transformada pela mudança de variáveis

$$
x=\frac{\eta \eta^{\prime}}{\eta^{\prime \prime}}, \quad y=\frac{\eta^{\prime 2}}{\eta \eta^{\prime \prime}}, \quad t=\ln \left|\eta^{\prime}\right|
$$

no sistema

$$
\begin{aligned}
& \dot{x}=x(1+x+y) \\
& \dot{y}=y(2+x-y) .
\end{aligned}
$$


que também pode ser transformado em um sistema da forma (3.1)-(3.2).

Para uma referência sobre a utilização de sistemas gerais da forma (3.3)-(3.4) no estudo de fenômenos físicos ver [10].

Nesta seção estuda-se, via compactificação, o efeito das perturbações periódicas sobre o sistema (3.1)-(3.2). A consideração de perturbações periódicas na análise de fenômenos naturais como os descritos acima (interação entre espécies, problemas da Astrofísica, Dinâmica dos Fluidos), é natural, dentro de cada contexto. Veja, por exemplo, [26] e referências ali citadas.

Conforme visto na seção 2.2, do Capítulo 2, a expressão (2.6) do campo compactificado não é válida no polo norte da esfera (ou equivalentemente, no centro $(0,0)$ do disco $\mathrm{D}^{2}$ ), pois a compactificação usando coordenadas polares não é válida neste ponto. Assim, como a solução do sistema (3.1)-(3.2), contida no eixo- $y$, passa pela origem do plano, que corresponde ao centro do disco, para evitar este ponto problemático vamos fazer uma translação da reta invariante do sistema para o ponto $(1,0)$. Com isso, a reta $\{\mathrm{x}=1\}$ passa a ser invariante, e o sistema (3.1)-(3.2) é então escrito da forma abaixo, após renomear-se as variáveis e constantes:

$$
\begin{aligned}
& \dot{x}=x y-y \\
& \dot{y}=\alpha+m x+n y+a x^{2}+b x y+c y^{2} .
\end{aligned}
$$

Vale então o seguinte resultado:

\section{Proposição 3.1.1 Sob as hipóteses}

$$
\begin{aligned}
& \text { H1) a) }(b+n)^{2}-4(\alpha+m+a) c<0 \text { e b) } m^{2}-4 a \alpha>0 \\
& \text { H2) } 0<c<1 \\
& H 3) b^{2}-4 a(c-1)<0,
\end{aligned}
$$

o sistema (3.5)-(3.6) possui somente dois pontos de equilibrio no infinito, que são pontos de sela hiperbólicos, $\theta_{1}$ e $\theta_{2}$. conectados por uma órbita heteroclínica $\gamma(s)$, que é uma 
reparametrização da solução $\varphi(t)$, contida na reta invariante $\{x=1\}$, formando os ciclos singulares infinitos $\gamma(s) \cup \theta_{1} \cup \pm \alpha(s) \cup \theta_{2}$ (Figura 3.1). Além disso, o campo possui dois pontos de equilíbrio no eixo-x, que sâo focos ou centros.

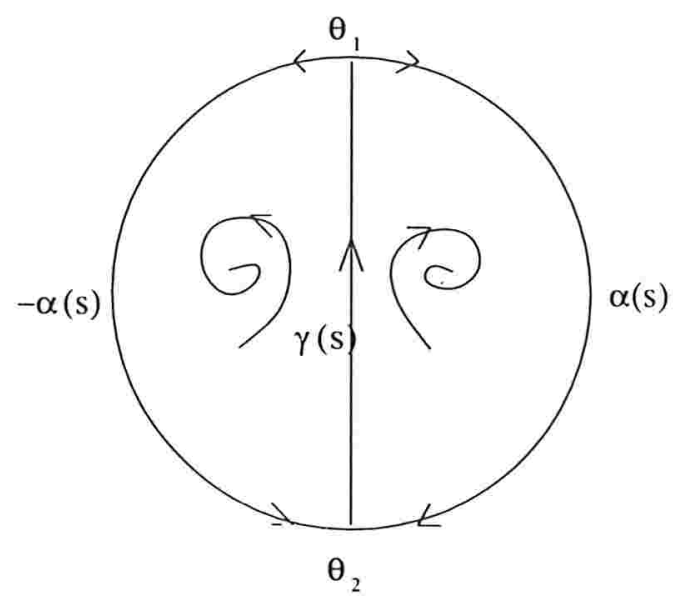

Figura 3.1: Ciclos singulares infinitos, simétricos, de um campo polinomial, no disco de Poincaré, com dois equilíbrios na parte compacta, que são poços.

Prova: Usando a expressão (2.6) do Capítulo 2, o compactificado de (3.5)-(3.6), escrito nas coordenadas $(\theta, \rho)$, é dado por:

$$
\begin{aligned}
& \dot{\theta}=X^{1}(\theta, \rho) \\
& \dot{\rho}=X^{2}(\theta, \rho)
\end{aligned}
$$

onde

$$
\begin{array}{r}
X^{1}(\theta, \rho)=(c-1) c(\theta) s^{2}(\theta)+a c^{3}(\theta)+b s(\theta) c^{2}(\theta)+ \\
+\rho s^{2}(\theta)+\rho m c^{2}(\theta)+\rho n s(\theta) c(\theta)+\rho^{2} \alpha c(\theta), \\
X^{2}(\theta, \rho)=-\rho\left[(a+1) c^{2}(\theta) s(\theta)+b c(\theta) s^{2}(\theta)+c s^{3}(\theta)+\right. \\
\left.+\rho(m-1) s(\theta) c(\theta)+\rho n s^{2}(\theta)+\rho^{2} \alpha s(\theta)\right] .
\end{array}
$$


$\operatorname{com} c(\theta)=\cos \theta$ e $s(\theta)=\sin \theta$.

Fazendo $\rho=0$ no sistema acima obtem-se

$$
\dot{\theta}=0 \Longleftrightarrow(c-1) \cos (\theta) \operatorname{sen}^{2}(\theta)+a \cos ^{3}(\theta)+b \operatorname{sen}(\theta) \cos ^{2}(\theta)=0,
$$

donde, da hipótese (H3), segue que $(\pi / 2,0)$ e $(3 \pi / 2.0)$ são os únicos pontos singulares de (3.7)-(3.8) no bordo do disco $\mathrm{D}^{2}$ (que correspondem aos pontos críticos de (3.5)-(3.6) no infinito). Calculando a jacobiana do campo nestes pontos, obtem-se

$$
J(\pi / 2,0)=\left(\begin{array}{cc}
1-c & 1 \\
0 & -c
\end{array}\right),
$$

$\mathrm{e}$

$$
J(3 \pi / 2,0)=\left(\begin{array}{cc}
c-1 & 1 \\
0 & c
\end{array}\right),
$$

o que, com a hipótese (H2), implica que eles são selas hiperbólicas.

De (H1-a) tem-se que a reta invariante $\{x=1\}$ não contém pontos de equilíbrio. Fazendo-se $x=1$ e integrando a equação obtem-se a seguinte solução, contida na reta $\{x=1\}$ :

$$
\varphi(t)=\left(1, \delta \operatorname{tg}\left(c \delta t+\operatorname{arctg}\left(\frac{n+b}{\delta}\right)\right)-(n+b)\right)=\left(\varphi_{1}(t), \varphi_{2}(t)\right),
$$

onde $\delta=\frac{1}{2 c} \sqrt{4 c(\alpha+m+a)-(n+b)^{2}}$. Tal solução está definida no intervalo

$$
\left(t_{-}, t_{+}\right)=\left(-\frac{\pi}{2 c \delta}-\frac{1}{c \delta} \operatorname{arctg}\left(\frac{n+b}{\delta}\right), \frac{\pi}{2 c \delta}-\frac{1}{c \delta} \operatorname{arctg}\left(\frac{n+b}{\delta}\right)\right) .
$$

Usando as relações entre $(x, y)$ e $(\theta, \rho)$, dadas por (conforme Observação 2.2.4. Capítulo 2)

$$
x=\frac{\cos \theta}{\rho} \quad y=\frac{\operatorname{sen} \theta}{\rho} \quad e \quad \rho=\frac{1}{\sqrt{x^{2}+y^{2}}}
$$

tem-se $\theta=\operatorname{arctg}(y / x)$, com o que pode-se encontrar a expressão da solução $\varphi(t)$ nas coordenadas $(\theta, \rho)$, que é dada por

$$
\gamma(s)=(\theta(s), \rho(s))=\left(\operatorname{arctg}\left(\varphi_{2}(t)\right), \frac{1}{\sqrt{1+\varphi_{2}(t)^{2}}}\right),
$$


onde $s=s(t)$ é o tempo no Disco de Poincaré, dado pelo Lema 2.2.3, sendo que $\lim _{t \rightarrow t_{ \pm}} s(t)= \pm \infty$. Assim, da expressão de $\varphi(t)$ acima tem-se que, quando $t \rightarrow t_{+}$, $\varphi_{2}(t) \rightarrow+\infty$, donde $\theta(s) \rightarrow \frac{\pi}{2}$ e $\rho(s) \rightarrow 0$; também. quando $t \rightarrow t_{-}, \varphi_{2}(t) \rightarrow-\infty$, $\theta(s) \rightarrow \frac{3 \pi}{2}$ e $\rho(s) \rightarrow 0$. Portanto, por unicidade, $\gamma(s)$ é uma reparametrização da solução $\varphi(t)$, contida na reta $\{x=1\}$.

Finalmente, de (H1-b) segue que o campo possui dois pontos de equilíbrio no eixo- $x$, que são, obviamente, centros ou focos (atratores ou repulsores). Concluindo, o esboço do retrato de fase do campo no disco de Poincaré tem a forma apresentada na Figura 3.1 (no caso em que os equilíbrios são poços).

Considere agora a seguinte perturbação periódica (não-autônoma) do sistema (3.5)(3.6)

$$
\begin{aligned}
& \dot{x}=x y-y+\mu+A\left(a_{1} \cos \tilde{\omega} t+a_{2} \operatorname{sen} \tilde{\omega} t\right) \\
& \dot{y}=\alpha+m x+n y+a x^{2}+b x y+c y^{2}+A g(\tilde{\omega} t),
\end{aligned}
$$

onde $g$ é uma função de classe $C^{k}, k \geq 2$, periódica de período $T=2 \pi / \tilde{\omega}$, sendo $\tilde{\omega}=\omega \sqrt{x^{2}+y^{2}}$, com $\omega>0$ constante. Trata-se de uma combinação da perturbação autônoma dada pela função $(1,0)$ com a perturbação periódica $\left(a_{1} \cos \tilde{\omega} t+\right.$ $\left.a_{2} \operatorname{sen} \tilde{\omega} t, g(\tilde{\omega} t)\right)$, relacionadas aos parâmetros $\mu$ e $A$. respectivamente. Observe que o período da perturbação periódica depende da posição $(x, y)$.

A perturbação acima é a mais natural possível, pois a parte autônoma é ortogonal a solução contida na reta $\{x=1\}$, que corresponde à órbita heteroclínica $\gamma(s)$ do campo compactificado. Como visto no Capítulo 2 , os pontos de sela no infinito, $\theta_{1}$ e $\theta_{2}$, do sistema não perturbado (3.5)-(3.6), passam a ser órbitas periódicas no infinito, $\theta_{1}(s)$ e $\theta_{2}(s)$, para o sistema perturbado (3.9)-(3.10). O parâmetro $\mu$ cumpre o papel de "separar" (ou desconetar) as variedades invariantes locais das órbitas periódicas $\theta_{1}(s)$ e $\theta_{2}(s)$, que dependem de $\mu$ e $A$, e as quais denotamos $W_{\mu, A}^{s}\left(\theta_{1}(s)\right)$ e $W_{\mu, A}^{u}\left(\theta_{2}(s)\right)$. O parâmetro $A$, por sua vez. representa a amplitude da perturbação periódica. O 
teorema abaixo estabelece condições sobre os parâmetros $A$ e $\mu$ para que as variedades $W_{\mu, A}^{s}\left(\theta_{1}(s)\right)$ e $W_{\mu, A}^{u}\left(\theta_{2}(s)\right)$ se tangenciem, se intersectem transversalmente e se separem, no espaço de fase estendido.

Teorema 3.1.2 Suponha que para $\mu=A=0$ o sistema (3.9)-(3.10) satisfaz as hipóteses (H1)-(H3) da Proposição 3.1.1. Então o sistema possui duas órbitas periódicas hiperbólicas, $\theta_{1}(s)$ e $\theta_{2}(s)$, no infinito, com variedades invariantes locais $W_{\mu, A}^{s}\left(\theta_{1}(s)\right)$ e $W_{\mu, A}^{u}\left(\theta_{2}(s)\right)$, e existem funções diferenciáveis $A_{M}(\mu)$ e $A_{m}(\mu)$, para $\mu$ suficientemente pequeno, tais que (ver Figura 3.2):

a) se $A=A_{M}(\mu)$ ou $A=A_{m}(\mu)$, as as variedades invariantes $W_{\mu, A}^{s}\left(\theta_{1}(s)\right)$ e $W_{\mu, A}^{u}\left(\theta_{2}(s)\right)$ se tangenciam quadraticamente;

b) se $\mu>0$ ( $\mu<0$, respectivamente) e $A_{m}(\mu)<A<A_{M}(\mu)\left(A_{M}(\mu)<A<A_{m}(\mu)\right.$, respectivamente), então $W_{\mu, A}^{s}\left(\theta_{1}(s)\right) \cap W_{\mu, A}^{u}\left(\theta_{2}(s)\right)=\emptyset$;

c) se $\mu>0$ e $A>A_{M}(\mu)$ ou $A<A_{m}(\mu)$, então $W_{\mu, A}^{s}\left(\theta_{1}(s)\right)$ e $W_{\mu, A}^{u}\left(\theta_{2}(s)\right)$ se intersectam transversalmente;

d) se $\mu<0$ e $A>A_{m}(\mu)$ ou $A<A_{M}(\mu)$, vale o mesmo resultado descrito em $\mathbf{c}$ ).

Observação: Com base no enunciado do teorema acima, o diagrama de bifurcação do sistema perturbado, numa vizinhança da origem no plano- $(\mu, A)$, é como o mostrado na Figura 3.2: existem dois tipos de regiões, $S_{1}$ e $S_{2}$, separadas pelas curvas $A_{M}(\mu)$ e $A_{m}(\mu)$, tais que, se o par $(\mu, A)$ encontra-se sobre estas curvas, o sistema apresenta tangências heteroclínicas; se $(\mu, A)$ pertence à região $S_{1}$, então $W_{\mu, A}^{s}\left(\theta_{1}(s)\right) \cap$ $W_{\mu, A}^{u}\left(\theta_{2}(s)\right)=\emptyset$; se $(\mu, A)$ pertence à região $S_{2}^{\prime}, W_{\mu, A}^{s}\left(\theta_{1}(s)\right)$ e $W_{\mu, A}^{u}\left(\theta_{2}(s)\right)$ se intersectam transversalmente em pelo menos um ponto.

A existência das tangências heteroclínicas e das intersecções heteroclínicas transversais entre as variedades $W_{\mu, A}^{s}\left(\theta_{1}(s)\right)$ e $W_{\mu, A}^{u}\left(\theta_{2}(s)\right)$ implicam na existência de pontos heteroclínicos transversais e tangências heteroclínicas das variedades invariantes de um ponto fixo da transformação de Poincaré associada ao campo. 


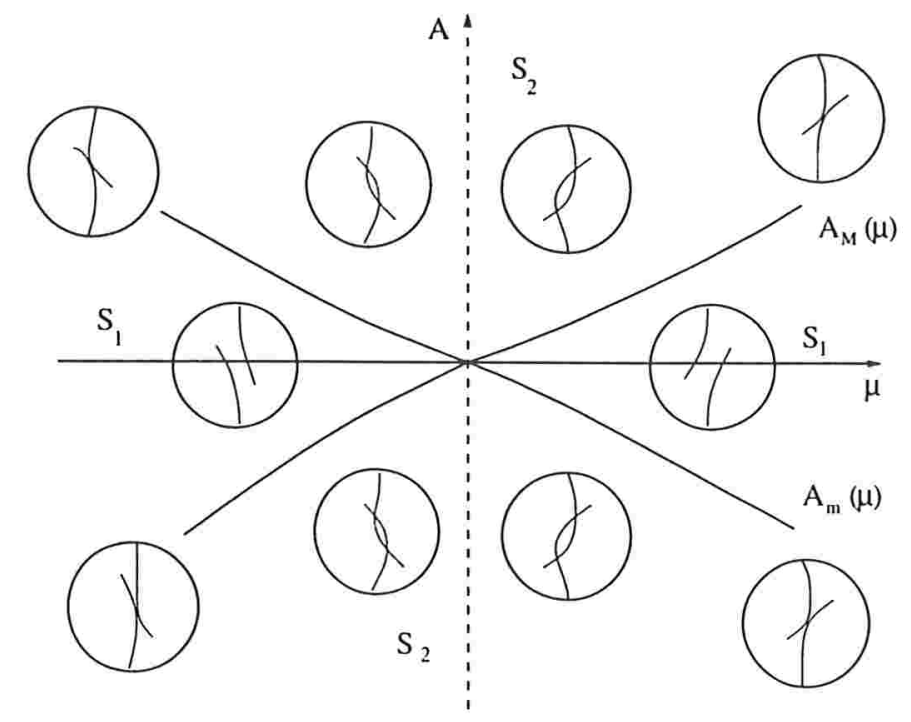

Figura 3.2: Diagrama de bifurcação do sistema (3.9)-(3.10) no plano- $(\mu, A)$. As figuras mostram a posição das variedades invariantes $W_{\mu, A}^{s}\left(\theta_{1}(s)\right)$ e $W_{\mu, A}^{u}\left(\theta_{2}(s)\right)$ em uma seção transversal ao toro $\mathrm{D}^{2} \times \mathrm{S}^{1}$.

São conhecidos da literatura resultados no contexto do teorema acima para perturbações periódicas de campos vetoriais contendo órbitas homoclínicas e heteroclínicas, contidas em regiões compactas do plano. O resultado acima estende a teoria existente para ciclos heteroclínicos infinitos, que não podem ser vistos em regiões compactas do plano. Em tais regiões o que se vê é apenas uma parte regular de tais soluções.

Conforme comentários tecidos no Capítulo 1, a existência de um ponto heteroclínico transversal para um difeomorfismo (no caso dado pela transformação de Poincaré associada ao sistema perturbado) implica na existência de infinitos pontos heteroclínicos transversais, o que ocorre também para os pontos de tangência quadrática. Do teorema acima segue que, variando-se o parâmetro $A$ na direção vertical, desde $A=0$, observa-se o seguinte cenário (ver Figura 3.2 e também a Figura 1.3, no Capítulo 1): se $A<A_{M}(\mu)$, as variedades $W_{\mu, A}^{s}\left(\theta_{1}(s)\right)$ e $W_{\mu, A}^{u}\left(\theta_{2}(s)\right)$ estão separadas; se $A=A_{M}(\mu)$, as variedades se tangenciam quadraticamente em um ponto (e, consequentemente, em infinitos pontos); se $A>A_{M}(\mu)$, as variedades se intersectam transversalmente em um 
ponto (e, consequentemente, em infinitos pontos). A Figura 1.3, mostrada no Capítulo 1, dá uma idéia do comportamento das variedades em uma seção transversal ao fluxo do sistema perturbado, para diferentes valores dos parâmetros $\mu$ e A. Tal comportamento implica em uma dinâmica bastante complexa das soluções do sistema perturbado no interior do toro sólidoD ${ }^{2} \times \mathrm{S}^{1}$ e, consequentemente, na parte finita do plano.

Prova do Teorema: Para simplificar a notação, omitiremos os parâmetros $(\mu, A)$ nas notações de $W_{\mu, A}^{s}\left(\theta_{1}(s)\right)$ e $W_{\mu, A}^{u}\left(\theta_{2}(s)\right)$. No decorrer da prova, usamos as construções elaboradas no Capítulo 2.

Compactificando (3.9)-(3.10), usando coordenadas polares $(\theta, \rho)$. conforme os cálculos desenvolvidos no Capítulo 2 (expressão 2.6), obtem-se a seguinte expressão

$$
\begin{aligned}
\dot{\theta} & =X_{p}^{1}(\theta, \rho, \phi, \epsilon) \\
\dot{\rho} & =X_{p}^{2}(\theta, \rho, \phi, \epsilon) \\
\dot{\phi} & =X_{p}^{3}(\theta, \rho, \phi, \epsilon),
\end{aligned}
$$

onde

$$
\begin{aligned}
X_{p}^{1}(\theta, \rho, \phi, \epsilon) & =(c-1) c(\theta) s^{2}(\theta)+a c^{3}(\theta)+b s(\theta) c^{2}(\theta)+\rho s^{2}(\theta) \\
& +\rho m c^{2}(\theta)+\rho n s(\theta) c(\theta)+\rho^{2} \alpha c(\theta)-\mu \rho^{2} s(\theta)-A \rho^{2} \cos \phi s(\theta), \\
X_{p}^{2}(\theta, \rho, \phi, \epsilon) & =-\rho\left[(a+1) c^{2}(\theta) s(\theta)+b c(\theta) s^{2}(\theta)+c s^{3}(\theta)\right. \\
& \left.+\rho(m-1) s(\theta) c(\theta)+\rho n s^{2}(\theta)+\rho^{2} \alpha s(\theta)-\mu \rho^{2} c(\theta)-A \rho^{2} \cos \phi s(\theta)\right], \\
X_{p}^{3}(\theta, \rho, \phi, \epsilon) & =\omega,
\end{aligned}
$$

sendo que $c(\theta)=\cos \theta, s(\theta)=\sin \theta$. O campo acima está definido no toro sólido $\left\{D^{2} \times S^{1}\right\}-\left\{(0,0) \times S^{1}\right\}$, sendo que os pontos no bordo deste toro correspondem aos pontos no infinito do campo (3.9)-(3.10).

Os pontos singulares no infinito, $\theta_{1}=(\pi / 2,0)$ e $\theta_{2}=(3 \pi / 2,0)$, que, por hipótese, existem para $A=\mu=0$ (conforme Proposição 3.1.1), correspondem, neste espaço de fase estendido, às órbitas periódicas

$$
\theta_{1}(s)=\left\{\left(\pi / 2,0, \omega s+\phi_{0}\right) \mid s \in \mathbb{R} \text { e } \phi_{0} \in[0,2 \pi)\right\}
$$




$$
\theta_{2}(s)=\left\{\left(3 \pi / 2,0, \omega s+\phi_{0}\right) \mid s \in \mathbb{R} \text { e } \phi_{0} \in[0,2 \pi)\right\}
$$

do sistema (3.11)-(3.13), com variedades invariantes $W^{s}\left(\theta_{1}(s)\right)$ e $W^{u}\left(\theta_{2}(s)\right)$, que, para $\mu=A=0$, coincidem, formando a variedade bidimensional

$$
\Gamma_{\theta_{1} \theta_{2}}=\left\{\left(\gamma\left(s_{0}\right), \phi_{0}\right) \mid s_{0} \in \mathbb{R} \text { e } \phi_{0} \in[0.2 \pi)\right\},
$$

onde $\gamma(s)$ é a trajetória heteroclínica do sistema compactificado, que conecta os pontos singulares $\theta_{1}$ e $\theta_{2}$ no disco de Poincaré (Figura 3.1).

Conforme Capítulo 2, seção 2.4 , seja $d\left(s_{0}, \phi_{0} \cdot \mu . A\right)$ a função de separação entre $W^{u}\left(\theta_{2}(s)\right)$ e $W^{s}\left(\theta_{1}(s)\right)$, relativamente a um ponto $p \in \Gamma_{\theta_{1} \theta_{2}}$, em uma seção $\Sigma^{\phi_{0}}$, transversal ao fluxo do campo. A expansão desta função em Série de Taylor, nas variáveis $\mu$ e $A$, em torno de $(0,0)$, é dada por

$$
d\left(s_{0}, \phi_{0}, \mu, A\right)=\frac{1}{N} \frac{\partial d\left(s_{0}, \phi_{0}, 0,0\right)}{\partial \mu} \mu+\frac{1}{N} \frac{\partial d\left(s_{0} \cdot o_{0} \cdot 0,0\right)}{\partial \mathcal{A}} A+R\left(s_{0}, \phi_{0}, \mu, A\right),
$$

onde $S=\frac{\partial d\left(s_{0}, \phi_{0}, 0,0\right)}{\partial \mu}$ e $M\left(\phi_{0}\right)=\frac{\partial d\left(s_{0}, \phi_{0}, 0,0\right)}{\partial A}$ são as funções de Melnikov-Sotomayor, relativas à parte autônoma e à parte periódica da perturbaçào, respectivamente,

$$
R\left(s_{0}, \phi_{0}, \mu, A\right)=a\left(s_{0}, \phi_{0}, \mu, A\right) \mu^{2}+b\left(s_{0}, \phi_{0} \cdot \mu . A\right) \mu . A+c\left(s_{0}, \phi_{0}, \mu, A\right) A^{2},
$$

sendo $a, b, c$ funções analíticas de $(\mu, A)$, tais que

$$
a\left(s_{0}, \phi_{0}, 0,0\right)=b\left(s_{0}, \phi_{0}, 0,0\right)=c\left(s_{0}, \phi_{0}, 0,0\right)=0
$$

e $N=\left\|X^{\perp}\left(\gamma\left(s_{0}\right)\right)\right\|$, que é diferente de zero para $s_{0}$ finito. O parâmetro $\phi_{0}$ é tomado de acordo com a seção transversal $\Sigma^{\phi_{0}}$ considerada. que é definida por

$$
\Sigma^{\phi_{0}}=\left\{(\theta, \rho, \phi) \mid(\theta, \rho) \in[0,2 \pi) \times[0,+\infty) \in \phi=\phi_{0} \in[0,2 \pi)\right\} .
$$

Vejamos as propriedades das funções $S$ e $M\left(\phi_{0}\right)$ acima.

Usando a expressão (2.14) obtida na seção 2.5. Capítulo 2, tomando-se $s_{0}=0 \mathrm{e}$ fazendo $(f(\omega s), g(\omega s))=(1,0)$, obtem-se:

$$
S:=\frac{\partial d\left(\phi_{0}, 0,0\right)}{\partial \mu}=-\int_{-\infty}^{+\infty} e^{-\int_{0}^{s} \sigma(\tau) d \tau} \rho(s)^{3} \sum_{i=0}^{2} \rho(s)^{i} Q_{2-i}(\cos \theta(s), \operatorname{sen} \theta(s)) d s .
$$


Usando ainda a expressão (2.14) e considerando $(f(\omega s), g(\omega s))=\left(a_{1} \cos \left(\omega s+\phi_{0}\right)+\right.$ $\left.a_{2} \operatorname{sen}\left(\omega s+\phi_{0}\right), g\left(\omega s+\phi_{0}\right)\right)$ naquela expressão obtem-se que

$$
M\left(\phi_{0}\right):=\frac{\partial d\left(\phi_{0}, 0,0\right)}{\partial A}=\int_{-\infty}^{+\infty} e^{-\int_{0}^{s} \sigma(\tau) d \tau} \rho(s)^{3} F\left(\theta, \rho, s, \phi_{0}\right) d s
$$

onde

$$
\begin{array}{r}
F\left(\theta, \rho, s, \phi_{0}\right)=\left(a_{1} \cos \left(\omega s+\phi_{0}\right)+a_{2} \operatorname{sen}\left(\omega s+\phi_{0}\right)\right) \sum_{i=0}^{2} \rho(s)^{i} Q_{2-i}(\cos \theta(s), \operatorname{sen} \theta(s))+ \\
g\left(\omega s+\phi_{0}\right) \sum_{i=0}^{n} \rho(s)^{i} P_{n-i}(\cos \theta(s) \cdot \operatorname{sen} \theta(s)) .
\end{array}
$$

Nas duas integrais acima, tem-se que

$$
\sigma(s)=\sum_{i=0}^{2} \rho(s)^{i} A_{2-i}^{\prime}(\theta(s))-\sum_{i=0}^{2}(i+1) \rho(s)^{i} R_{2-i}(\theta(s)),
$$

sendo $A_{k}$ e $R_{k}$ as expressões dadas em (2.3) e (2.4), respectivamente, e $\gamma(s)=(\theta(s), \rho(s))$ é a expressão da conexão heteroclínica entre $\theta_{1}$ e $\theta_{2}$ no disco de Poincaré, dada pela Proposição 3.1 .1 , isto é

$$
\gamma(s)=(\theta(s), \rho(s))=\left(\operatorname{arctg}\left(\varphi_{2}(t)\right), \frac{1}{\sqrt{1+\varphi_{2}(t)^{2}}}\right),
$$

onde $\varphi(t)=\left(1, \varphi_{2}(t)\right)$ é a solução do campo polinomial no plano, contida na reta invariante $\{x=1\}$.

Considerando-se agora as relações (conforme observações 2.2 .2 e 2.2.4. do Capítulo 2),

$$
\theta=\operatorname{arctg}\left(\frac{y}{x}\right), \quad \rho=\frac{1}{\sqrt{x^{2}+y^{2}}} \quad e \quad \frac{d s}{d t}=\frac{1}{\rho^{n-1}},
$$

e o fato de $\gamma(s)$ ser uma reparametrizaçào da solução $\varphi(t)=\left(1, \varphi_{2}(t)\right)$ contida na reta $\{x=1\}$, tem-se, conforme a fórmula integral (2.16), obtida na seção 2.5. do Capítulo 2 , que a função $S$ obtida acima é dada, em coordenadas $(x, y)$ no plano, por

$$
S=-\int_{t_{-}}^{t_{+}} e^{-\int_{0}^{t} \tilde{\sigma}(\tau) d \tau}\left(1+\varphi_{2}(t)^{2}\right)^{-2} Q\left(1, \varphi_{2}(t)\right) d t
$$


Da hipótese (H1-a) da Proposição 3.1.1, tem-se $Q(1, y)>0 . \quad \forall y$, donde segue que o integrando na função acima é produto de funções positivas e, portanto, $S<0$.

Por outro lado, como para o campo polinomial $(P, Q)$ no plano tem-se $P(1, y)=$ $0, \forall y, M\left(\phi_{0}\right)$ obtida acima é dada, em coordenadas $(x, y)$ do plano, por (conforme expressão (2.16))

$$
M\left(\phi_{0}\right)=\int_{t_{-}}^{t_{+}} e^{-\int_{0}^{t} \tilde{\sigma}(\tau) d \tau}\left(1+\varphi_{2}(t)^{2}\right)^{-2} Q\left(1, \varphi_{2}(t)\right)\left[a_{1} \cos \left(\omega t+\phi_{0}\right)+a_{2} \operatorname{sen}\left(\omega t+\phi_{0}\right)\right] d t .
$$

Da expressão acima tem-se que $M\left(\phi_{0}\right)$ satisfaz a equação diferencial $M\left(\phi_{0}\right)=$ $-M^{\prime \prime}\left(\phi_{0}\right)$ para todo $\phi_{0}$ e, portanto, é da forma

$$
M\left(\phi_{0}\right)=B \operatorname{sen}\left(\omega t+\phi_{0}\right)+D \cos \left(\omega t+\phi_{0}\right)
$$

donde segue que tal função assume valores máximo e mínimo no intervalo $[0,2 \pi)$, com

$$
M\left(\phi_{\min }\right)<0<M\left(\phi_{\max }\right)
$$

sendo que os pontos críticos $\phi_{\min }$ e $\phi_{\max }$ são não-degenerados, isto é $M^{\prime \prime}\left(\phi_{\min }\right) \neq 0 \mathrm{e}$ $M^{\prime \prime}\left(\phi_{\max }\right) \neq 0$.

Consideremos então a expressão (3.14), com $s_{0}=0$, escrita na forma

$$
d\left(\phi_{0}, \mu, A\right)=\frac{S}{N} \mu+\frac{M\left(\phi_{0}\right)}{N} A+R\left(s_{0}, \phi_{0} \cdot \mu, A\right),
$$

onde $S$ e $M\left(\phi_{0}\right)$ foram calculadas acima. Colocando $\mu / N$ em evidência na expressão acima tem-se

$$
d\left(\phi_{0}, \mu, \eta\right)=\frac{\mu}{N}\left(S+M\left(\phi_{0}\right) \eta+\tilde{R}\left(s_{0}, \phi_{0} \cdot \mu, \eta\right)\right),
$$

onde $\eta=A / \mu \mathrm{e}$

$$
\tilde{R}\left(s_{0}, \phi_{0}, \mu, \eta\right)=a\left(s_{0}, \phi_{0}, \mu, \mu \eta\right) \mu+b\left(s_{0}, \phi_{0}, \mu . \mu \eta\right) \mu \eta+c\left(s_{0}, \phi_{0}, \mu, \mu \eta\right) \eta^{2} \mu,
$$

que é $O(\mu)$, para $\mu \rightarrow 0$.

Chamando

$$
\Delta\left(\phi_{0}, \mu, \eta\right):=S+M\left(\phi_{0}\right) \eta+\check{R}\left(s_{0} \cdot \phi_{0} \cdot \mu \cdot \eta\right)
$$


tem-se de (3.15) que

$$
d\left(\phi_{0}, \mu, \eta\right)=\frac{\mu}{N} \Delta\left(\phi_{0}, \mu, \eta\right),
$$

donde segue que, como $N>0$, para $\mu \neq 0$ suficientemente pequeno, $d\left(\phi_{0}, \mu, \eta\right)=0$ se, e somente se, $\Delta\left(\phi_{0}, \mu, \eta\right)=0$. Analisemos então a existência de zeros da função $\Delta$ definida acima, usando o Teorema da Função Implícita.

Primeiramente encontremos a região no espaço de parâmetros $(\mu, A)$ na qual as variedades invariantes locais $W_{\mu, A}^{s}\left(\theta_{1}(s)\right)$ e $W_{\mu, A}^{u}\left(\theta_{2}(s)\right)$ nào se intersectam, ou seja, $W_{\mu, A}^{s}\left(\theta_{1}(s)\right) \cap W_{\mu, A}^{u}\left(\theta_{2}(s)\right)=\emptyset$, com o que ficará provado o ítem (b) do Teorema.

Lembremos que, para as variedades invariantes locais das órbitas periódicas $\theta_{1}(s)$ e $\theta_{2}(s)$ se intersectarem, devemos ter

$$
\Delta\left(\phi_{0}, \mu, \eta\right):=S+M\left(\phi_{0}\right) \eta+\tilde{R}\left(\phi_{0}, \mu, \eta\right)=0
$$

Agora, como

$$
M\left(\phi_{\min }\right) \leq M\left(\phi_{0}\right) \leq M\left(\phi_{\max }\right)
$$

isolando $M\left(\phi_{0}\right)$ na expressão (3.16), tem-se que a condição sobre os parâmetros para que as variedades invariantes se intersectem é

$$
M\left(\phi_{\min }\right) \leq-\frac{S}{\eta}-\frac{O(\mu)}{\eta} \leq M\left(\phi_{\max }\right)
$$

onde o termo $O(\mu)=\tilde{R}\left(\phi_{0}, \mu, \eta\right)$. Como $S<0$ e $M\left(\phi_{\min }\right)<0<M\left(\phi_{\max }\right)$, as desigualdades acima implicam nas relações

$$
\eta \geq-\frac{S}{M\left(\phi_{\min }\right)}-O(\mu) \quad \text { ou } \quad \eta \geq-\frac{S}{M\left(\phi_{\max }\right)}-O(\mu) \quad \text { se } \eta>0
$$

$\mathrm{e}$

$$
\eta \leq-\frac{S}{M\left(\phi_{\min }\right)}-O(\mu) \quad \text { ou } \quad \eta \leq-\frac{S}{M\left(\phi_{\max }\right)}-O(\mu) \quad \text { se } \eta<0
$$

Dentre as quatro relações acima, $\eta \geq-\frac{S}{M\left(\phi_{\min }\right)}-O(\mu)$ e $\eta \leq-\frac{S}{M\left(\phi_{\max }\right)}-O(\mu)$ são verificadas, para $\mu$ suficientemente pequeno, devido às hipóteses. Usando as outras 
duas relações e voltando a mudança de variáveis $\eta=A / \mu$, obtem-se, para $\mu>0$,

$$
A \geq-\frac{S}{M\left(\phi_{\max }\right)} \mu-O\left(\mu^{2}\right) \quad e \quad A \leq-\frac{S}{M\left(\phi_{\min }\right)} \mu-O\left(\mu^{2}\right)
$$

Por outro lado, se $\mu<0$ obtem-se

$$
A \leq-\frac{S}{M\left(\phi_{\max }\right)} \mu-O\left(\mu^{2}\right) \quad e \quad A \geq-\frac{S}{M\left(\phi_{\min }\right)} \mu-O\left(\mu^{2}\right)
$$

As quatro relações acima, para o parâmetro $A$ como função de $\mu$, determinam as regiões no espaço de parâmetros, para as quais as variedades invariantes locais $W_{\mu, A}^{s}\left(\theta_{1}(s)\right)$ e $W_{\mu, A}^{u}\left(\theta_{2}(s)\right)$ se intersectam em pelo menos um ponto (região hachureada na Figura $3.3)$.

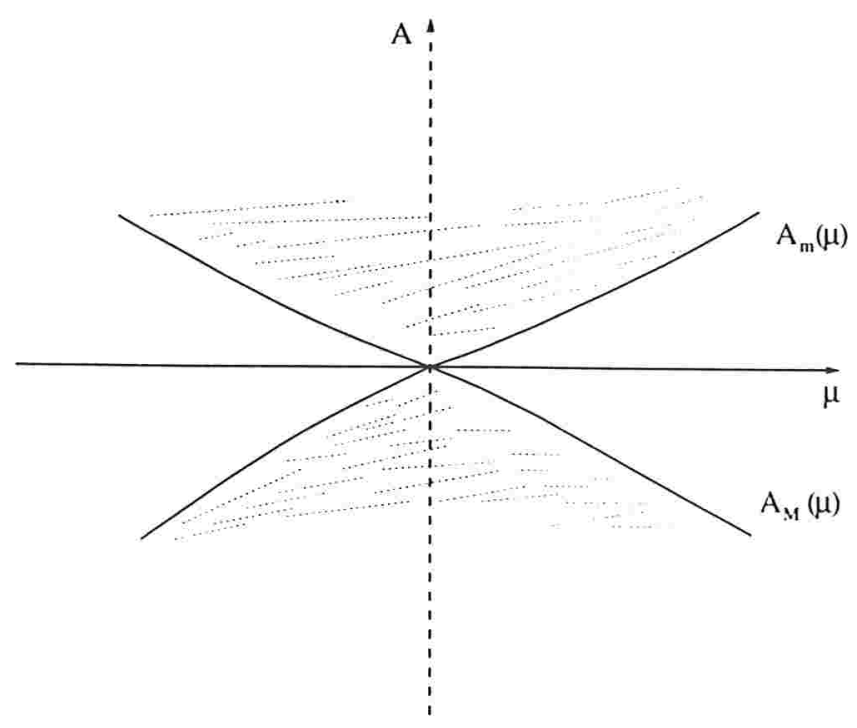

Figura 3.3: Regiōes no espaço de parametros $\mu$ e $A$ para as quais há intersecção entre as variedades $W_{\mu, A}^{s}\left(\theta_{1}(s)\right)$ e $W_{\mu, A}^{u}\left(\theta_{2}(s)\right)$.

Tais regiões têm como fronteira as curvas $A_{M}(\mu)$ e $A_{m}(\mu)$. com $\mu$ suficientemente pequeno, dadas por

$$
A_{M}(\mu)=-\frac{S}{M\left(\phi_{\max }\right)} \mu-O\left(\mu^{2}\right)
$$




$$
A_{m}(\mu)=-\frac{S}{M\left(\phi_{\min }\right)} \mu-O\left(\mu^{2}\right),
$$

que independem da seção transversal $\Sigma^{\phi_{0}}$, onde está definida a função de separação. Dependem apenas dos valores de $S, M\left(\phi_{\min }\right)$ e $M\left(\phi_{\max }\right)$.

Dos cálculos acima conclui-se que a interseç̧ão entre as variedades $W_{\mu, A}^{s}\left(\theta_{1}(s)\right)$ e $W_{\mu, A}^{u}\left(\theta_{2}(s)\right)$ é vazia se, e somente se,

$$
A_{M}(\mu)<A<A_{m}(\mu), \quad \text { para } \mu>0
$$

ou

$$
A_{m}(\mu)<A<A_{M}(\mu), \quad \text { para } \mu<0,
$$

o que corresponde ao complementar da região hachureada na Figura 3.3. Fica assim provado o ítem (b) do Teorema.

Mostremos agora que se os parâmetros $(\mu, A)$ estão na fronteira da região acima, ou seja, sobre as curvas $A_{m}(\mu)$ ou $A_{M}(\mu)$, então as variedades se tangenciam quadraticamente e, se o par $(\mu, A)$ pertence ao interior da região (parte hachureada da Figura $3.3)$, as variedades se intersectam transversalmente.

Considere a função $\Delta\left(\phi_{0}, \mu, \eta\right)$, definida acima por

$$
\Delta\left(\phi_{0}, \mu, \eta\right)=S+M\left(\phi_{0}\right) \eta+O(\mu)
$$

onde $O(\mu)=\tilde{R}\left(\phi_{0}, \mu, \eta\right), S$ e $M\left(\phi_{0}\right)$ são as integrais de Melnikov-Sotomayor obtidas acima.

Tomando-se

$$
\bar{\eta}=-\frac{S}{M\left(\bar{\phi}_{0}\right)},
$$

$\operatorname{com} M\left(\bar{\phi}_{0}\right) \neq 0$, tem-se dois casos a considerar

Caso 1) $\bar{\phi}_{0}=\phi_{\max }$ ou $\bar{\phi}_{0}=\phi_{\min }$. Neste caso, considerando-se $\bar{\phi}_{0}=\phi_{\max }$, temos

$$
\text { i) } \Delta\left(\phi_{\max }, 0, \bar{\eta}\right)=0
$$




$$
\begin{aligned}
& \text { ii) } \frac{\partial \Delta}{\partial \phi_{0}}\left(\phi_{\max }, 0, \bar{\eta}\right)=\bar{\eta} M^{\prime}\left(\phi_{\max }\right)=0 \\
& \text { iii) } \frac{\partial^{2} \Delta}{\partial \phi_{0}^{2}}\left(\phi_{\max }, 0, \bar{\eta}\right)=S<0 \\
& \text { iv) } \frac{\partial \Delta}{\partial \eta}\left(\phi_{\max }, 0, \bar{\eta}\right)=M\left(\phi_{\max }\right)>0 .
\end{aligned}
$$

De (i) e (iv) segue, do Teorema da Função Implícita, que, para $\left(\phi_{0}, \mu\right) \in V i z\left(\phi_{\max }\right) \times$ $V i z(0)$, existe uma superfície $\eta=\eta\left(\phi_{0}, \mu\right), \operatorname{com} \eta\left(\phi_{\max }, 0\right)=\bar{\eta}=-S / M\left(\phi_{\max }\right)$, tal que

$$
\Delta\left(\eta\left(\phi_{0}, \mu\right), \phi_{0}, \mu\right)=0
$$

donde tem-se que, para $\mu \neq 0$ suficientemente pequeno, $d\left(\phi_{0}, \mu, \eta\left(\phi_{0}, \mu\right)\right)=0$, ou seja, as variedade se intersectam em um ponto $\left(\gamma(0)+O(\mu), \phi_{\max }\right)$. Além disso, de (ii) e (iii) tem-se, pelo Teorema 2.4.4, do Capítulo 2 , que $W^{s}\left(\theta_{1}(s)\right)$ e $W^{u}\left(\theta_{2}(s)\right)$ se tangenciam quadraticamente no ponto $\left(\gamma(0)+O(\mu), \phi_{\max }\right)$ para $\eta=\bar{\eta}+O(\mu)$.

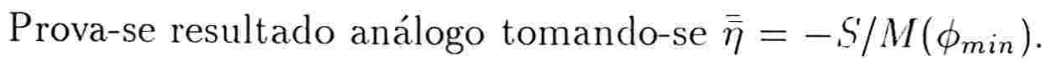

Voltando-se aos parâmetros originais, $\mu, A$, através da relação $\eta=A / \mu$. obtem-se, da relação $\eta=\bar{\eta}+O(\mu)$, com $\mu$ suficientemente pequeno, as curvas

$$
A_{M}(\mu)=-\frac{S}{M\left(\phi_{\max }\right)} \mu+O\left(\mu^{2}\right)
$$

e

$$
A_{m}(\mu)=-\frac{S}{M\left(\phi_{\min }\right)} \mu+O\left(\mu^{2}\right)
$$

no plano- $(\mu, A)$, para as quais $W^{s}\left(\theta_{1}(s)\right)$ e $W^{u}\left(\theta_{2}(s)\right)$ se tangenciam quadraticamente. Observe que, para $\mu$ suficientemente pequeno, pela unicidade da função obtida com o Teorema da Função Implícita, tais curvas correspondem à fronteira da região obtida anteriormente (Figura 3.3). 
Caso 2) $\bar{\phi}_{0} \neq \phi_{\max }$ e $\bar{\phi}_{0} \neq \phi_{\min }$. Neste caso tem-se

$$
\begin{aligned}
& \text { i) } \Delta\left(\bar{\phi}_{0}, 0, \bar{\eta}\right)=0 \\
& \text { ii) } \frac{\partial \Delta}{\partial \phi_{0}}\left(\bar{\phi}_{0}, 0, \bar{\eta}\right)=\bar{\eta} M^{\prime}\left(\bar{\phi}_{0}\right) \neq 0 \\
& \text { iii) } \frac{\partial \Delta}{\partial \eta}\left(\bar{\phi}_{0}, 0, \bar{\eta}\right)=M\left(\bar{\phi}_{0}\right)>0 .
\end{aligned}
$$

De (i) e (iii) segue, do Teorema da Função Implícita, que existe uma função $\eta_{1}=$ $\eta_{1}\left(\phi_{0}, \mu\right)$, para $\left(\phi_{0}, \mu\right) \in V i z\left(\bar{\phi}_{0}\right) \times V i z(0), \operatorname{com} \eta_{1}\left(\bar{\phi}_{0}, 0\right)=\bar{\eta}=-S / M\left(\bar{\phi}_{0}\right)$, tal que

$$
\Delta\left(\eta_{1}\left(\phi_{0}, \mu\right), \phi_{0}, \mu\right)=0
$$

donde tem-se que, para $\mu \neq 0$ suficientemente pequeno, $d\left(\phi_{0}, \mu, \eta_{1}\left(\phi_{0}, \mu\right)\right)=0$. Além disso, de (ii) tem-se, pelo Teorema 2.4.2 e Lema 2.4.3, que $W^{s}\left(\theta_{1}(s)\right)$ e $W^{u}\left(\theta_{2}(s)\right)$ se intersectam transversalmente no ponto $\left(\gamma(0)+O(\mu), \bar{\phi}_{0}\right)$, sendo que, devido a (iii), tal intersecção persiste para $\eta=\bar{\eta}+O(\mu)$.

Voltando-se a mudança de variáveis $\eta=A / \mu$ obtem-se as curvas

$$
A_{1}(\mu)=-\frac{S}{M\left(\bar{\phi}_{0}\right)} \mu+O\left(\mu^{2}\right)
$$

e

$$
A_{2}(\mu)=-\frac{S}{M\left(\bar{\phi}_{0}\right)} \mu+O\left(\mu^{2}\right)
$$

no plano- $(\mu, A)$, para as quais $W^{s}\left(\theta_{1}(s)\right)$ e $W^{u}\left(\theta_{2}(s)\right)$ se intersectam transversalmente, em pelo menos um ponto.

Usando o fato de que $M\left(\phi_{\min }\right)<M\left(\bar{\phi}_{0}\right)<M\left(\phi_{\max }\right)$, tem-se, para $\mu$ suficientemente pequeno,

$$
A_{1}(\mu)>A_{M}(\mu) \quad e \quad A_{2}(\mu)<A_{m}(\mu),
$$

donde segue que o par $(A, \mu)$ encontra-se na região hachureada da Figura 3.3. 
Mais ainda, as desigualdades acima são válidas para todo $\bar{\phi}_{0}$ tal que $\phi_{\min }<\bar{\phi}_{0}<$ $\phi_{\max }$, desde que $M\left(\bar{\phi}_{0}\right) \neq 0$. Assim, pode-se concluir que o coeficiente angular da reta tangente às curvas $A_{1}$ e $A_{2}$ acima, em $\mu=0$, tende, por um lado, para $\infty$ quando $\bar{\phi}_{0} \rightarrow \phi_{0}$, com $M\left(\phi_{0}\right)=0$, e, por outro lado, tende para o coeficiente angular das curvas de tangências, $A_{M}(\mu)$ e $A_{m}(\mu)$, quanto $\bar{\phi}_{0} \rightarrow \phi_{\max }$ e $\bar{\phi}_{0} \rightarrow \phi_{\min }$, respectivamente.

Conclui-se então que se $\mu>0$ e $A>A_{M}(\mu)$ ou $A<A_{m}(\mu)$ (região hachureada na Figura 3.3), as variedades invariantes $W^{s}\left(\theta_{1}(s)\right)$ e $W^{u}\left(\theta_{2}(s)\right)$ se intersectam transversalmente em pelo menos um ponto, para $\mu$ suficientemente pequeno.

A prova do caso $\mu<0$ é feita de modo inteiramente análogo, observando-se o sinal das funções.

Observação: Se no sistema (3.9)-(3.10) considerarmos $\tilde{\omega}=\omega=$ constante, então sua compactificação toma a forma

$$
\begin{aligned}
& \dot{\theta}=\sum_{i=1}^{n} \rho^{i} A_{n-i}(\theta)+\epsilon \bar{f}(\theta, \rho, \phi) \\
& \dot{\rho}=-\sum_{i=0}^{n} \rho^{i+1} R_{n-i}(\theta)+\epsilon \bar{g}(\theta, \rho, \phi) \\
& \dot{\phi}=\omega \rho,
\end{aligned}
$$

$\operatorname{com}(\theta, \rho) \in[0,2 \pi) \times[0,+\infty), \phi \in \mathrm{S}^{1}$ e $\omega>0$.

Assim, em vez de duas órbitas periódicas no infinito ("casca" do toro sólido), o sistema compactificado passa a ter duas "linhas de singularidades" no infinito, dadas por:

$$
\lambda_{1}=\left\{(\pi / 2,0, \phi) \mid \phi \in \mathrm{S}^{1}\right\} \quad \text { e } \quad \lambda_{2}=\left\{(3 \pi / 2,0, \phi) \mid \phi \in \mathrm{S}^{1}\right\},
$$

sendo que, no infinito, as soluções do sistema são ortogonais às singularidades que formam as linhas $\lambda_{1}$ e $\lambda_{2}$ (ver Figura 3.4).

Este caso será estudado analíticamente por nós em um trabalho futuro. Porém, na próxima seção apresentamos algumas simulações numéricas de um sistema quadrático 

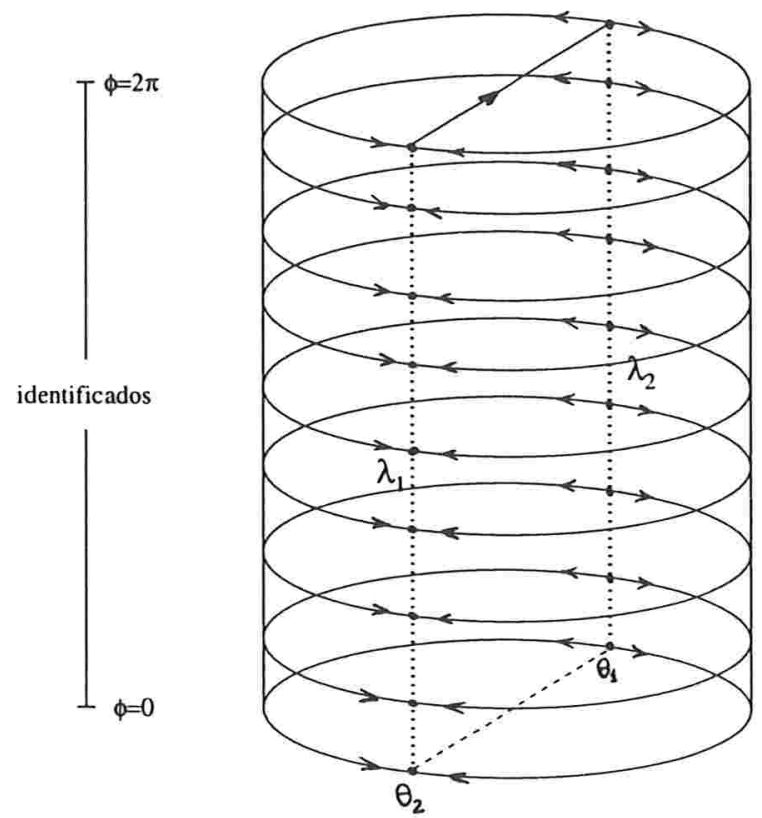

Figura 3.4: Linhas de singularidades no infinito do sistema (3.9)-(3.10), no caso em que $\tilde{\omega}$ é constante.

no plano, que apresenta a estrutura acima, que indicam ser bastante complexo o comportamento dinâmico das soluçõs do sistema perturbado, também neste caso.

\subsection{Simulações numéricas}

\section{Exemplo 3.2.1}

Considere o sistema

$$
\begin{aligned}
& \dot{x}=x y+\mu+A \text { sent } \\
& \dot{y}=1-y-x^{2}+\frac{1}{2} y^{2} .
\end{aligned}
$$

Para $\mu=A=0$, o sistema acim satisfaz as hipóteses H1-H3 da Proposição 3.1.1. Assim, tal sistema possui uma solução contida no eixo- $y$, que é invariante, conectando dois pontos de sela hiperbólicos no infinito (conforme as definições dadas anteriormente). Verifica-se, com alguns cálculos simples, que o sistema possui dois pontos de equilíbrio no eixo- $x$, mais precisamente os pontos $(1,0)$ e $(-1,0)$, que são focos atratores. Além 
disso, como $\tilde{\omega}=1$, o sistema apresenta duas linhas de singularidades no infinito, conforme a observação acima.

Na Figura 3.5 (a) é mostrado o plano de fase do sistema. com $\mu=A=0$, onde pode-se observar as características descritas acima.

Nas figuras seguintes, mostramos alguns dos resultados obtidos com as simulações numéricas desenvolvidas, relativamente ao sistema perturbado, os quais descrevemos a seguir.

Nas Figuras 3.5 (b) a (d) e Figuras 3.6 (e) e (f), são mostradas as projeções no plano- $(x, y)$ das soluções do sistema perturbado, $\operatorname{com} \mu=0.01$ e valores de $A$ iguais a $0.1,0.4,0.49,0.5$ e 0.52 , respectivamente, com condições iniciais $(0,-100)$ e $(0,-0.5,-1)$ (no "futuro"), e $(0,100)$ (no "passado"). Tais projeções dão uma idéia do comportamento das soluções do sistema, ao mantermos $\mu=0.01$ fixo e variarmos o parâmetro $A$ : para $A=0.1$ e $A=0.4$ (Figuras (b) e (c)), as soluções estão separadas (tal comportamento se repete para $0.1<A<0.4$, conforme simulações desenvolvidas); para $A=0.49$ (Figura (d)), há indícios de que as soluções se tangenciam pela primeira vez; para $A=0.5$ e $A=0.52$ (Figuras (e) e (f)), as simulações efetuadas indicam que as soluções se intersectam tranversalmente.

A projeção da solução mostrada na Figura 3.6 (f) sugere uma dinâmica complexa para as soluções do sistema perturbado. Porém, uma análise mais detalhada, mostra que trata-se apenas de uma solução periódica do sistema. Com efeito. a Figura 3.6 (g) mostra as soluções no espaço de fase $(x, y, t)$, e na Figura 3.6 (h) plotamos as iteradas da transformação de Poincaré, determinada pelo fluxo do sistema perturbado no tempo $2 \pi$, com valores dos parâmetros $\mu=0.01$ e $A=0.52$. Seis mil iteradas foram plotadas, com a mesma condição inicial usada para plotar as Figuras $(\mathrm{f})$ e $(\mathrm{g})$. O gráfico formado pelas iteradas da Transformação de Poincaré sugerem que trata-se de uma órbita periódica do sistema perturbado.

Pelas simulações desenvolvidas, as projeções das soluções apresentam o mesmo padrào da figura (f) (mesmo para diferentes condições iniciais) para valores de $A$ entre 


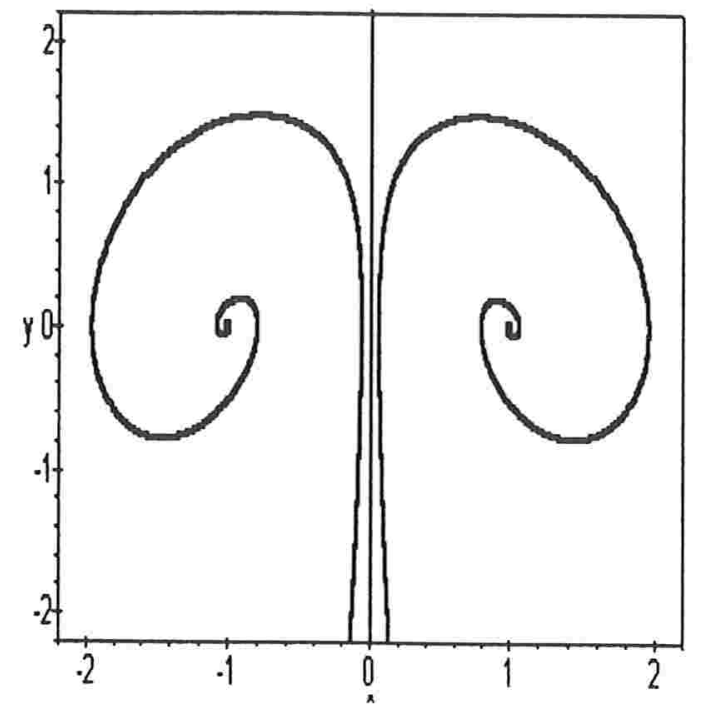

(a) $\quad \mu=0.0 \quad \mathrm{~A}=0.0$

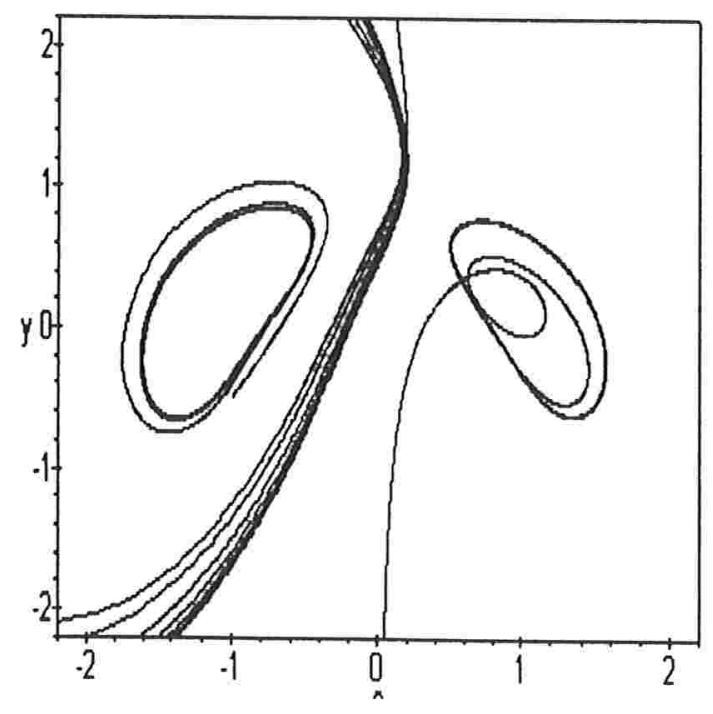

(c) $\quad \mu=0.01 \quad \mathrm{~A}=0.4$

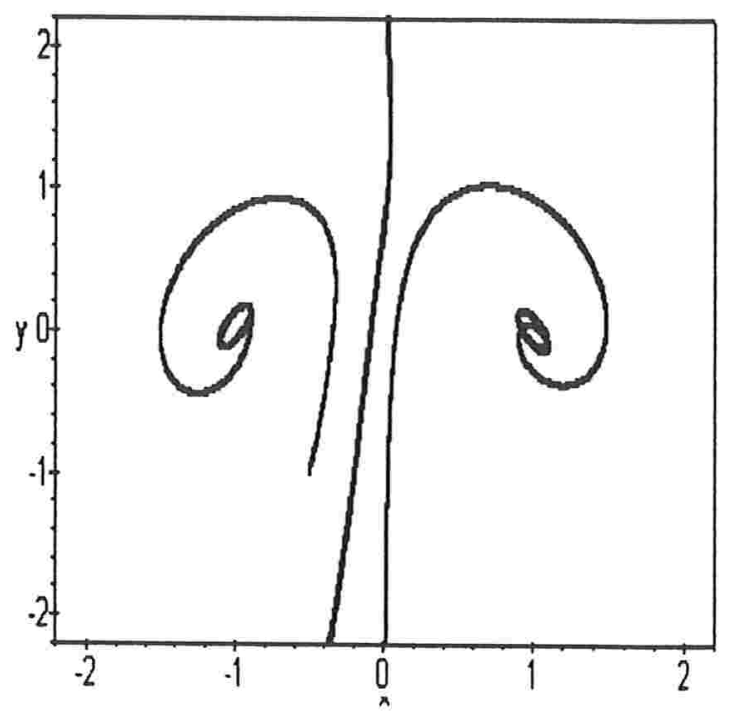

(b) $\quad \mu=0.01 \quad \mathrm{~A}=0.1$

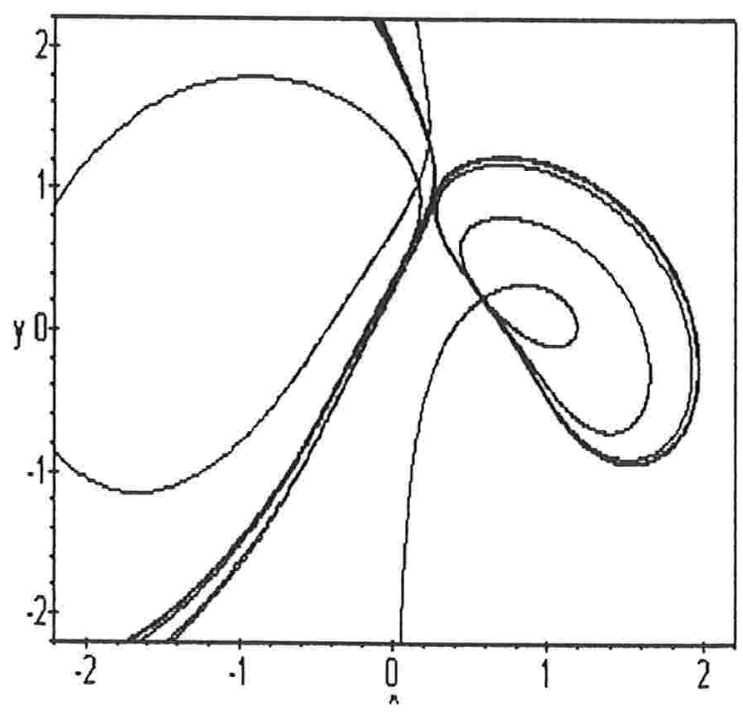

(d) $\quad \mu=0.01 \quad \mathrm{~A}=0.49$

Figura 3.5: Planos de fase do sistema do exemplo (3.2.1) para diferentes valores dos paràmetros. 


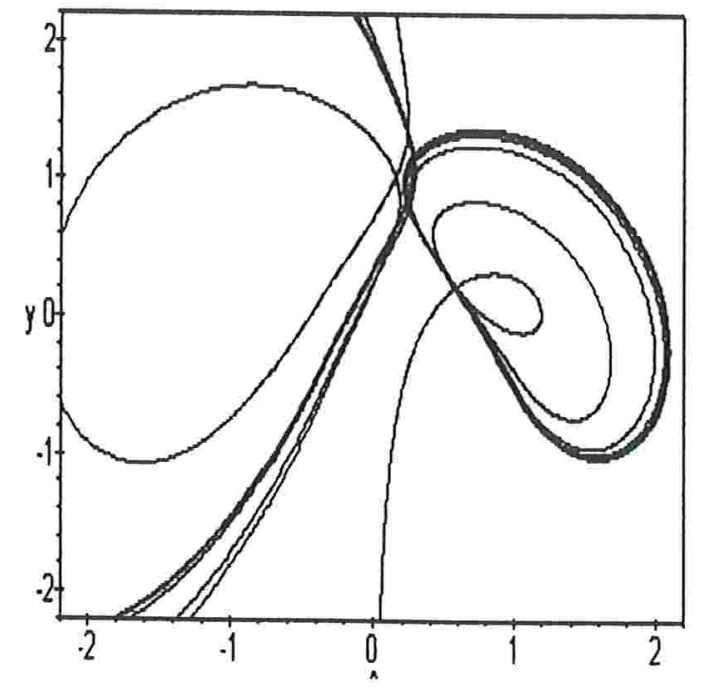

(e) $\quad \mu=0.01 \quad \mathrm{~A}=0.5$

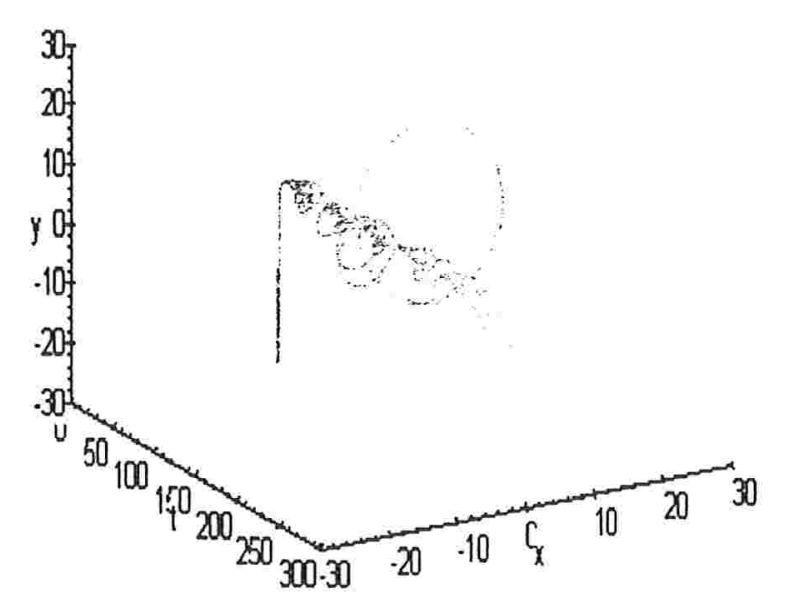

(g) $\quad \mu=0.01 \quad \mathrm{~A}=0.52$

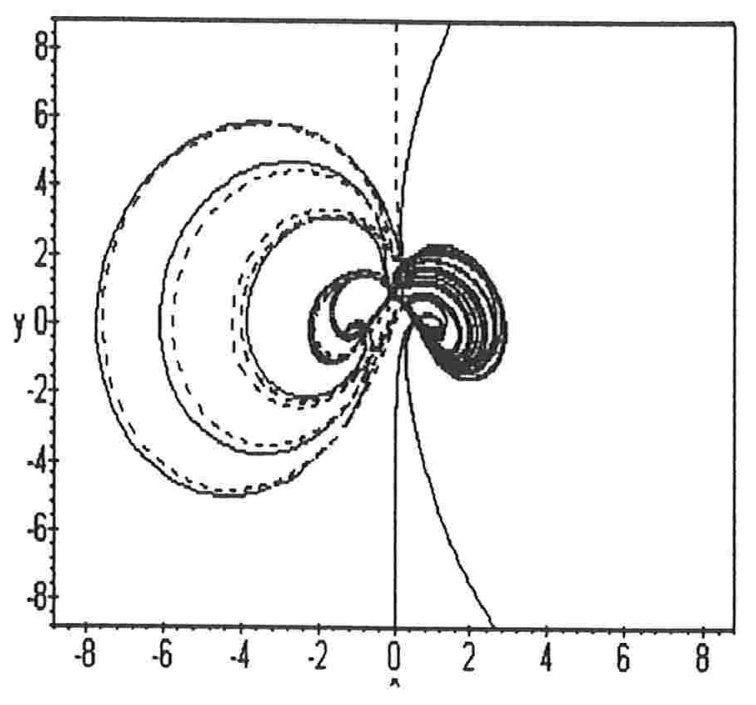

(f) $\quad \mu=0.01 \quad \mathrm{~A}=0.52$

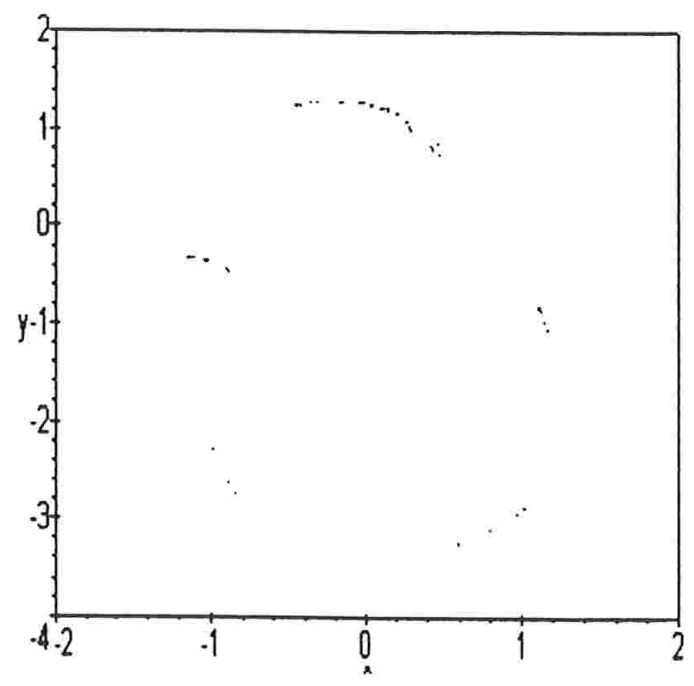

(h) $\quad \mu=0.01 \quad \mathrm{~A}=0.52$

Figura 3.6: Planos de fase do sistema do exemplo (3.2.1) para diferentes valores dos parâmetros. 


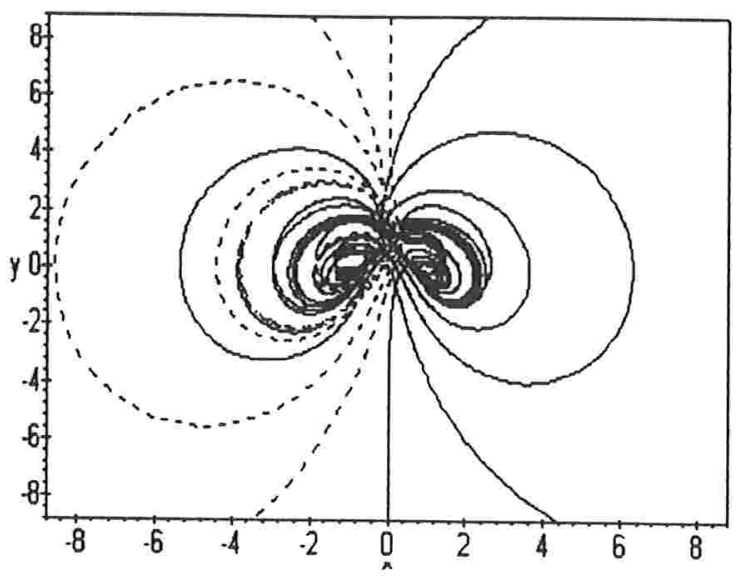

(i) $\quad \mu=0.01 \quad \mathrm{~A}=0.543$

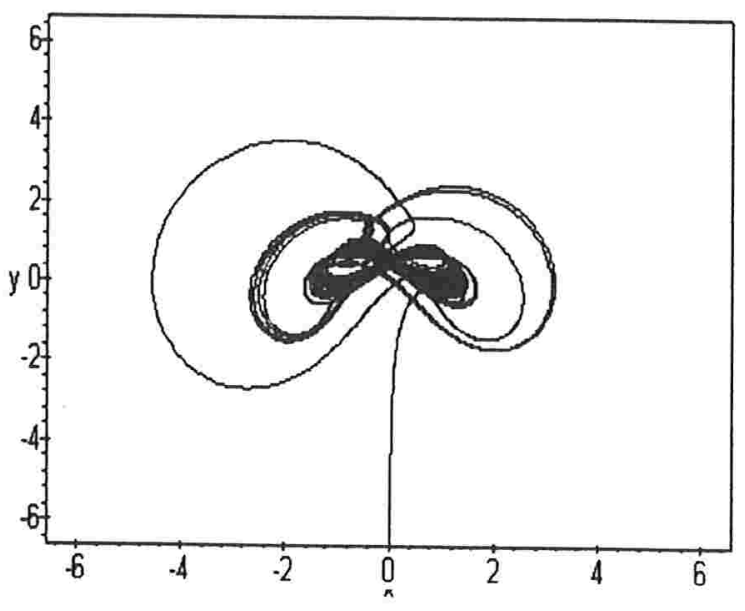

(k) $\quad \mu=0.01 \quad \mathrm{~A}=0.99$

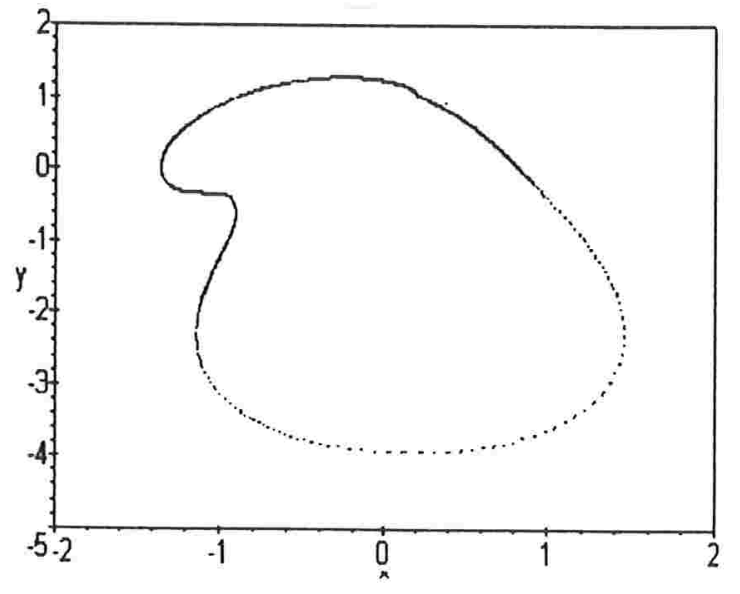

(j) $\mu=0.01 \quad \mathrm{~A}=0.543$

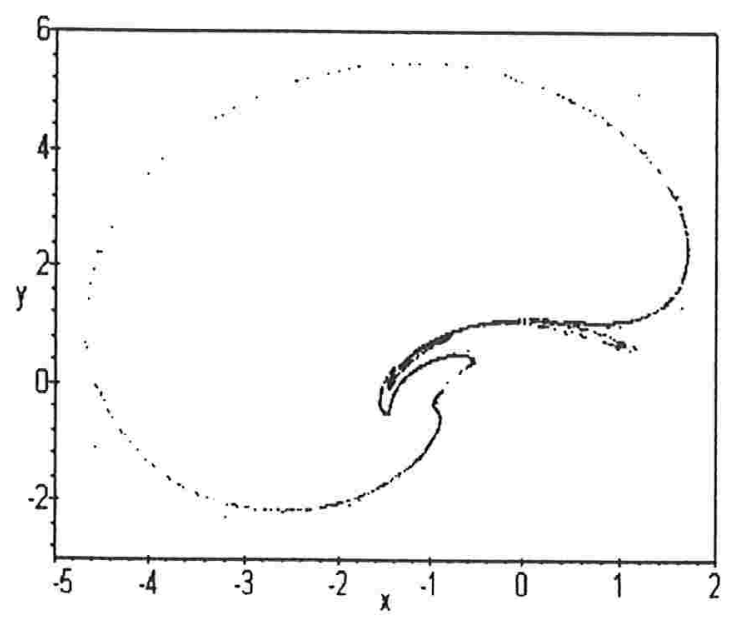

(l) $\mu=0.01 \quad \mathrm{~A}=0.99$

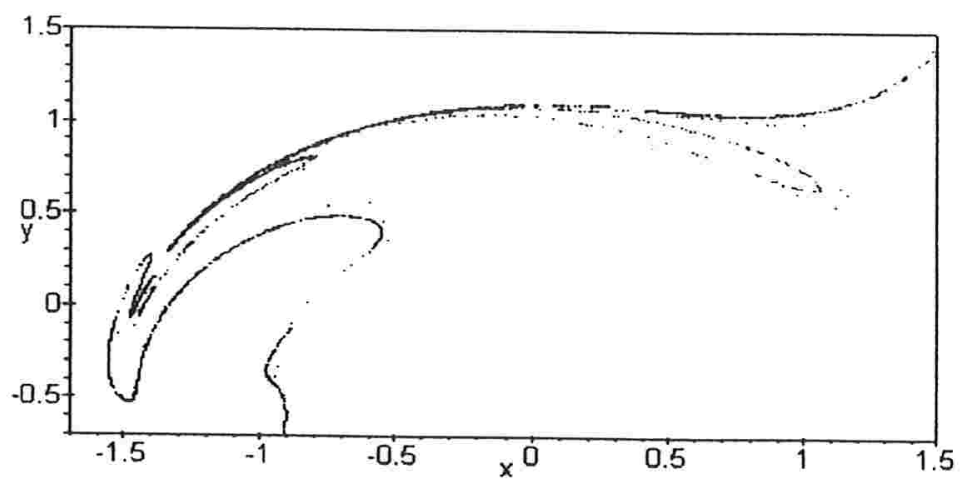

(m)

Figura 3.7: Planos de fase do sistema do exemplo (3.2.1) para diferentes valores dos paràmetros. 


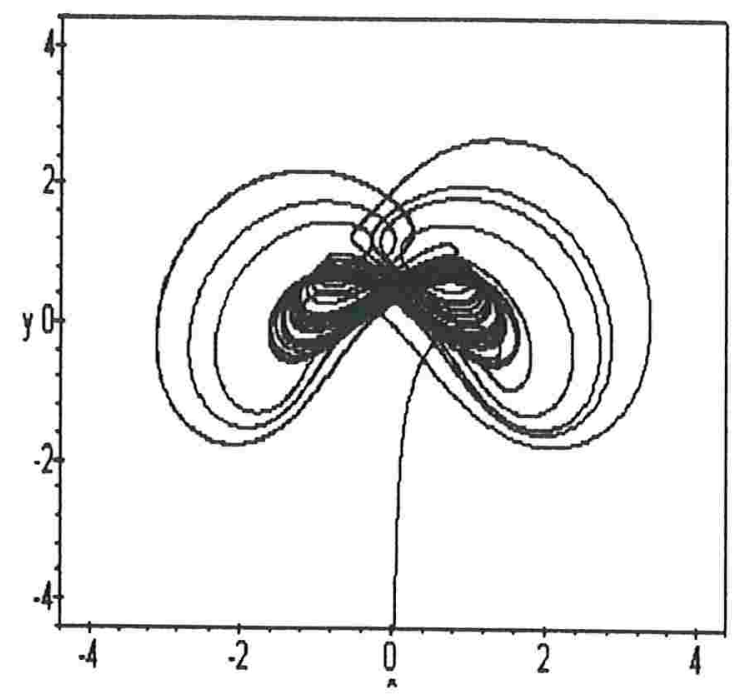

(n) $\quad \mu=0.01 \quad \mathrm{~A}=0.98$

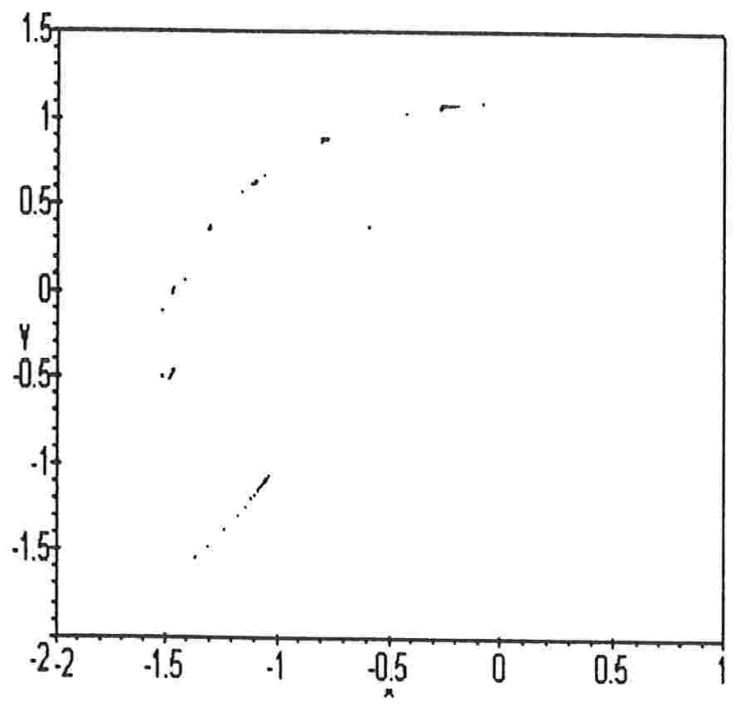

(p) $\mu=0.01 \quad \mathrm{~A}=0.981$

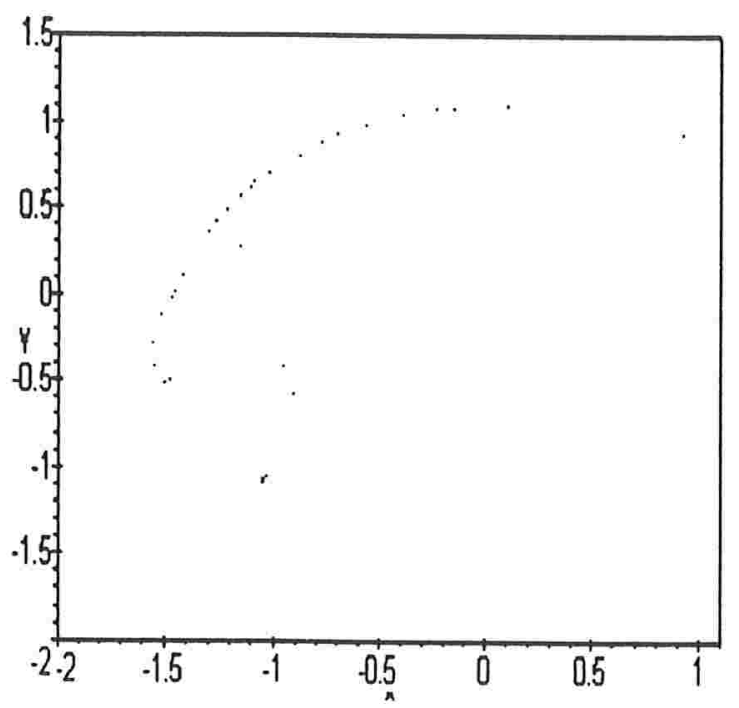

(o) $\quad \mu=0.01 \quad \mathrm{~A}=0.98$

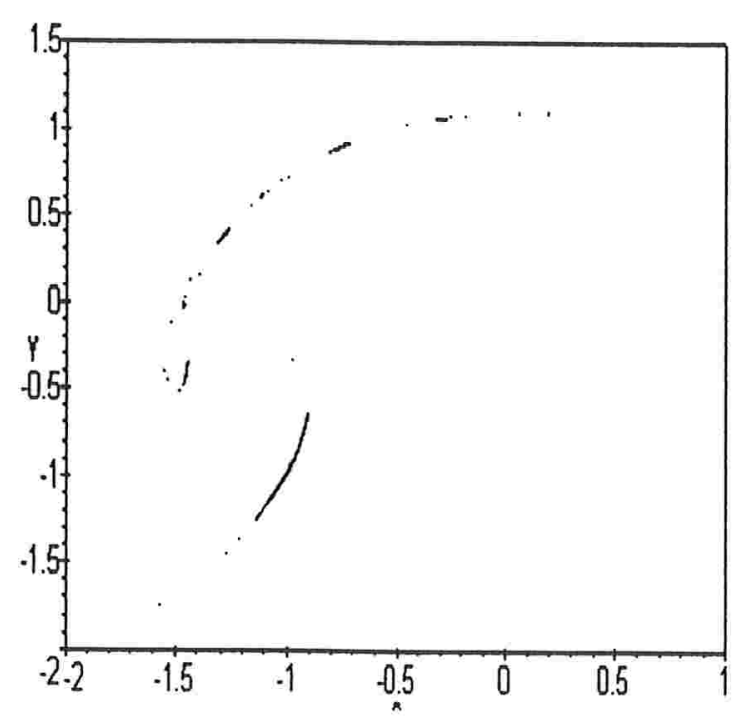

(q) $\quad \mu=0.01 \quad \mathrm{~A}=0.982$

Figura 3.8: Planos de fase do sistema do exemplo (3.2.1) para diferentes valores dos parámetros. 


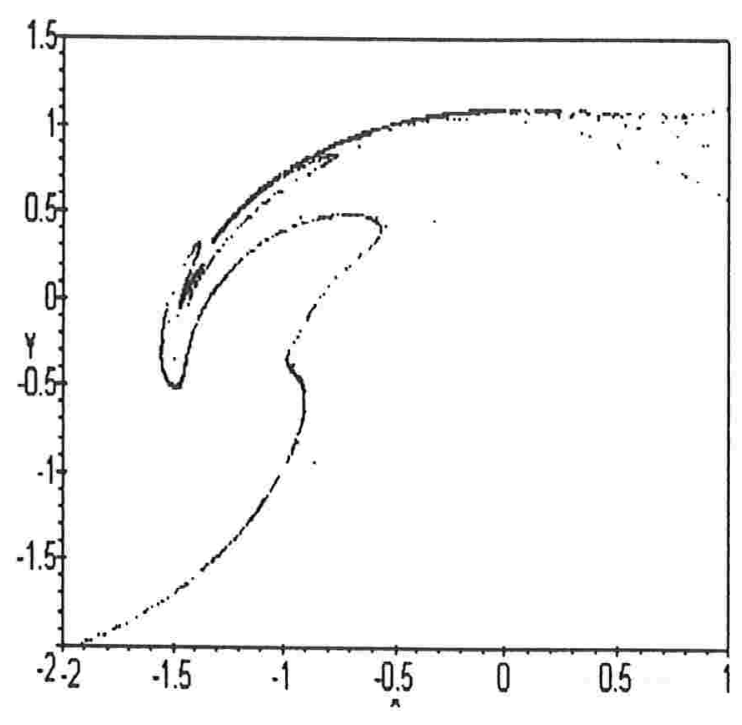

(r) $\mu=0.01 \quad \mathrm{~A}=0.985$

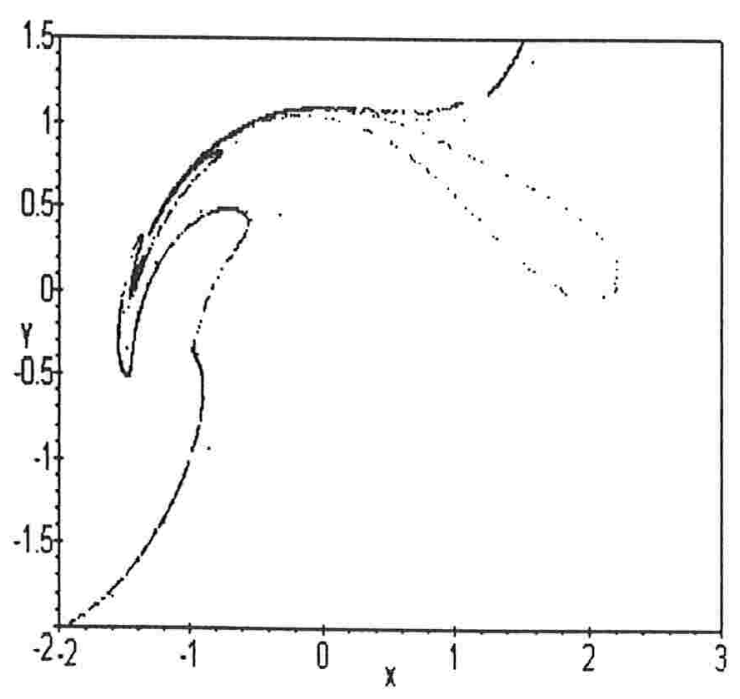

(t) $\quad \mu=0.01 \quad \mathrm{~A}=0.985$

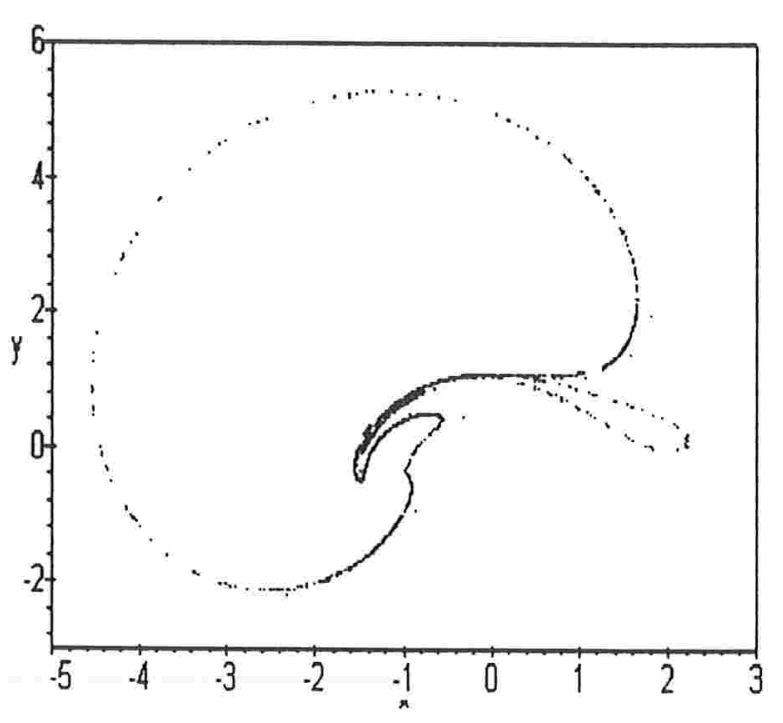

(s) $\quad \mu=0.01 \quad \mathrm{~A}=0.985$

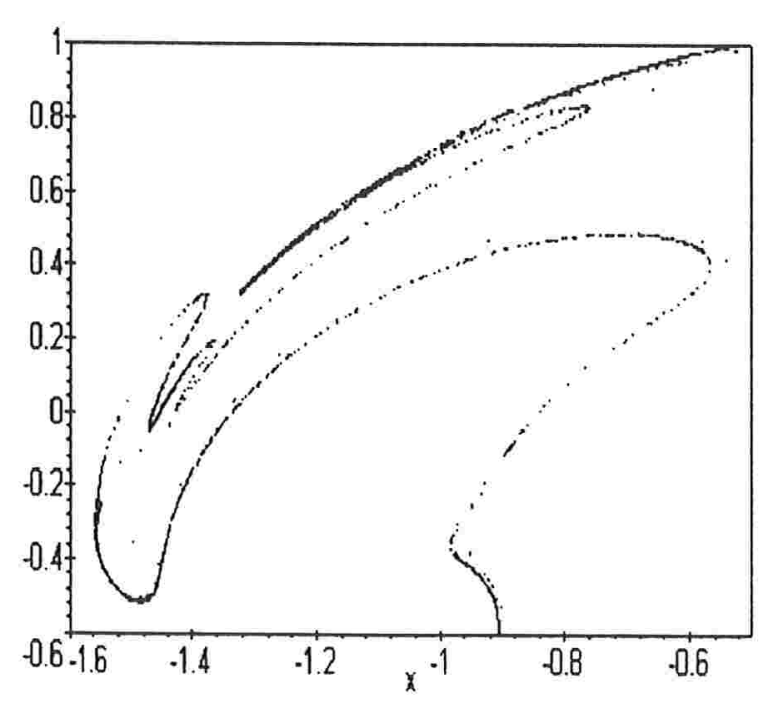

(u) $\mu=0.01 \quad \mathrm{~A}=0.985$

Figura 3.9: Planos de fase do sistema do exemplo (3.2.1) para diferentes valores dos paràmetros. 
0.5 e 1. Porém a transformação de Poincaré associada muda drásticamente.

De fato, a projeção das soluções no plano- $(x, y)$, para $A=0.543$ e $\mu=0.01$, é mostrada na Figura 3.i (i). As iteradas da transformação de Poincaré (fluxo no tempo $2 \pi$ ) associada apresenta uma curva invariante, que sugere a existência de um toro invariante para o sistema perturbado.

Agora, para $A=0.99$, algo diferente ocorre: as projeções mantém o padrão da Figura 3.6 (f), porém a tranformação de Poincaré dada pelo fluxo do sistema perturbado no tempo $2 \pi$ apresenta um tipo de "atrator", mostrado na figura 3.7 (1), com uma ampliação da parte mais "cheia" do atrator mostrada na figura (m). Esta última figura foi obtida plotando-se 7.500 iteradas da transformação de Poincaré, com condição inicial $(x, y)=(0,-100)$. Obtem-se a mesma figura para condições iniciais diferentes, tomadas aleatoriamente.

As Figuras 3.8 e 3.9 (n) a (u) dão uma idéia de como surge o atrator supostamente existente, ao variar-se o parâmetro $A$ : primeiro existe o comportamento periódico das soluções (Figura (o)); a quantidade de pontos periódicos da Transformação de Poincaré vai aumentando até que surgem curvas desconexas de pontos; finalmente se forma o atrator conexo, conforme mostrado nas figuras (r) a (u). Tal comportamento é também observado na formação do atrator de Hénon (conforme [1]).

As simulações numéricas desenvolvidas sugerem que o atrator descrito acima, para $\mu=0.01, A=0.99$, e condição inicial $(0,-100)$, continua existindo para quase toda condição inicial considerada.

\subsection{Um resultado mais geral}

Considere agora uma perturbação periódica mais geral da família (3.5)-(3.6), da forma

$$
\begin{aligned}
& \dot{x}=x y-y+\epsilon f(\tilde{\omega} t) \\
& \dot{y}=\alpha+m x+n y+a x^{2}+b x y+c y^{2}+\epsilon g(\dot{\omega} t),
\end{aligned}
$$


onde $f$ e $g$ são funções de classe $C^{k}, k \geq 2 \mathrm{em} \mathbb{R}$, periódicas de período $T=2 \pi / \tilde{\omega}$, sendo $\tilde{\omega}=\omega \sqrt{x^{2}+y^{2}}$, com $\omega>0$ constante. Suponha que $f$ seja suficientemente diferenciável para que possamos considerar seu desenvolvimento em Série de Fourier, dado por:

$$
f(\tilde{\omega} t)=\sum_{n=0}^{\infty}\left(a_{n} \cos (n \tilde{\omega} t)+b_{n} \operatorname{sen}(n \tilde{\omega} t)\right) .
$$

Suponha ainda que o termo $a_{0}$ da expansão de f seja distinto de zero, de maneira que possamos escrever

$$
f(\tilde{\omega} t)=a_{0}+\sum_{n=1}^{\infty}\left(a_{n} \cos (n \tilde{\omega} t)+b_{n} \operatorname{sen}(n \tilde{\omega} t)\right)=a_{0}+\tilde{f}(\tilde{\omega} t) .
$$

Substituindo-se a expressão acima no sistema (3.17)-(3.18), obtem-se

$$
\begin{aligned}
& \dot{x}=x y-y+\epsilon\left(a_{0}+\tilde{f}(\tilde{\omega} t)\right) \\
& \dot{y}=\alpha+m x+n y+a x^{2}+b x y+c y^{2}+\epsilon g(\tilde{\omega} t) .
\end{aligned}
$$

Chamando $\mu=\epsilon a_{0}$, o sistema acima toma a forma

$$
\begin{aligned}
& \dot{x}=x y-y+\mu+\epsilon \tilde{f}(\tilde{\omega} t) \\
& \dot{y}=\alpha+m x+n y+a x^{2}+b x y+c y^{2}+\epsilon g(\tilde{\omega} t)
\end{aligned}
$$

Observe o fato que na expressão acima o parâmetro $\mu$, que desempenha o papel da parte autônoma da perturbação. que separa as variedades, aparece naturalmente, quando o termo constante da expansào da funçào periódica $f(\tilde{\omega} t)$ em Série de Fourier é não-nulo. Assim, sem esforço adicional. usando-se a mesma técnica da prova do Teorema 3.1 .2 , prova-se o seguinte resultado, onde considera-se uma perturbação periódica mais geral da família (3.5)-(3.6):

Teorema 3.3.1 Suponha que, para $\mu=\epsilon=0$ a família de sistemas polinomiais quadráticos $X_{\mu, \epsilon}$ dada em (3.20)-(3.21) satisfaz as hipóteses (H1)-(H3) da Proposição 3.1.1. Considere a funçào

$$
M\left(\phi_{0}\right)=\int_{t_{-}}^{t_{+}} \epsilon^{-\int_{0}^{t} \tilde{\sigma}(\tau) d \tau}\left(1+\varphi_{2}(t)^{2}\right)^{-2} Q(\varphi(t)) f\left(\omega t+\phi_{0}\right) d t
$$


onde $\varphi(t)$ é a solução de $X_{\mu, \epsilon}$, com $\mu=\epsilon=0$, contida na reta invariante $\{x=1\}$. Se $M\left(\phi_{0}\right)$ possui $k$ pontos críticos nâo degenerados, $\phi_{1}, \phi_{2}, \cdots, \phi_{k}$, em $[0,2 \pi)$, com $M\left(\phi_{i}\right) \neq M\left(\phi_{j}\right)$ para $i \neq j$, e $M\left(\phi_{i}\right) \neq 0, \forall i$, então existe uma vizinhança da origem no espaço de parâmetros, contendo curvas $\epsilon_{i}(\mu), i=1,2, \cdots, k$, tais que, se o par $(\mu, \epsilon) \in \epsilon_{i}(\mu)$, o sistema perturbado apresenta tangências heteroclínicas quadráticas entre as variedades invariantes $W_{\mu, \epsilon}^{s}\left(\theta_{1}(s)\right)$ e $W_{\mu, \epsilon}^{u}\left(\theta_{2}(s)\right)$ das órbitas periódicas de (3.20)(3.21) no infinito. Além disso, dentre as curvas acima, existem duas curvas, $\epsilon_{m}(\mu) e$ $\epsilon_{M}(\mu)$, que dividem uma vizinhança do espaço de parâmetros em quatro regiões disjuntas, $S_{1}, S_{2}, S_{3}, S_{4}$, tais que (Figura 3.10):

i) se $(\mu, \epsilon) \in S_{1}$ ou $(\mu, \epsilon) \in S_{2}$, então $W_{\mu, \epsilon}^{u}\left(\theta_{2}(s)\right) \cap W_{\mu, \epsilon}^{s}\left(\theta_{1}(s)\right)=\emptyset$;

ii) as curvas $\epsilon_{i}(\mu), \quad i=1,2, \cdots, k$, com $i \neq m$ e $i \neq M$, estão contidas nas regiões $S_{3}$ e $S_{4}$, sendo que, se $M\left(\phi_{i}\right)>0$, então $\epsilon_{i}(\mu) \in S_{3}$ e se $M\left(\phi_{i}\right)<0, \epsilon_{i}(\mu) \in S_{4}$;

iii) se $(\mu, \epsilon) \in S_{3}$ ou $(\mu, \epsilon) \in S_{4}$, então $W_{\mu, \epsilon}^{u}\left(\theta_{2}(s)\right)$ e $W_{\mu, \epsilon}^{s}\left(\theta_{1}(s)\right)$ se intersectam, sendo tal interseç̧ão:

a) tangência quadrática, se $(\mu, \epsilon) \in \epsilon_{i}(\mu)$, para algum $i$, com $1<i<k$, ou

b) transversal, se $(\mu, \epsilon) \notin \epsilon_{i}(\mu)$.

Prova: A prova do teorema acima é feita seguindo passo a passo a prova do Teorema 3.1 .2 , para cada um dos pontos críticos $\phi_{i}$, e levando em consideração os seguints detalhes:

1) como $M(\phi)$ é uma função diferenciável, periódica em $[0,2 \pi)$, ela assume valores máximo e mínimo globais neste intervalo; chamando tais valores $\phi_{\max }$ e $\phi_{\min }$, respectivamente, e supondo-se, sem perda de generalidade, que $M\left(\phi_{\min }\right)<0<M\left(\phi_{\max }\right)$, tem-se, seguindo a prova do Teorema 3.1.2, que as curvas $\epsilon_{M}(\mu)$ e $\epsilon_{m}(\mu)$, do enunciado, são dadas por

$$
\epsilon_{M}(\mu)=-\frac{S}{M\left(\phi_{\max }\right)} \mu-O\left(\mu^{2}\right)
$$

e

$$
\epsilon_{m}(\mu)=-\frac{S}{M\left(\phi_{\min }\right)} \mu-O\left(\mu^{2}\right)
$$




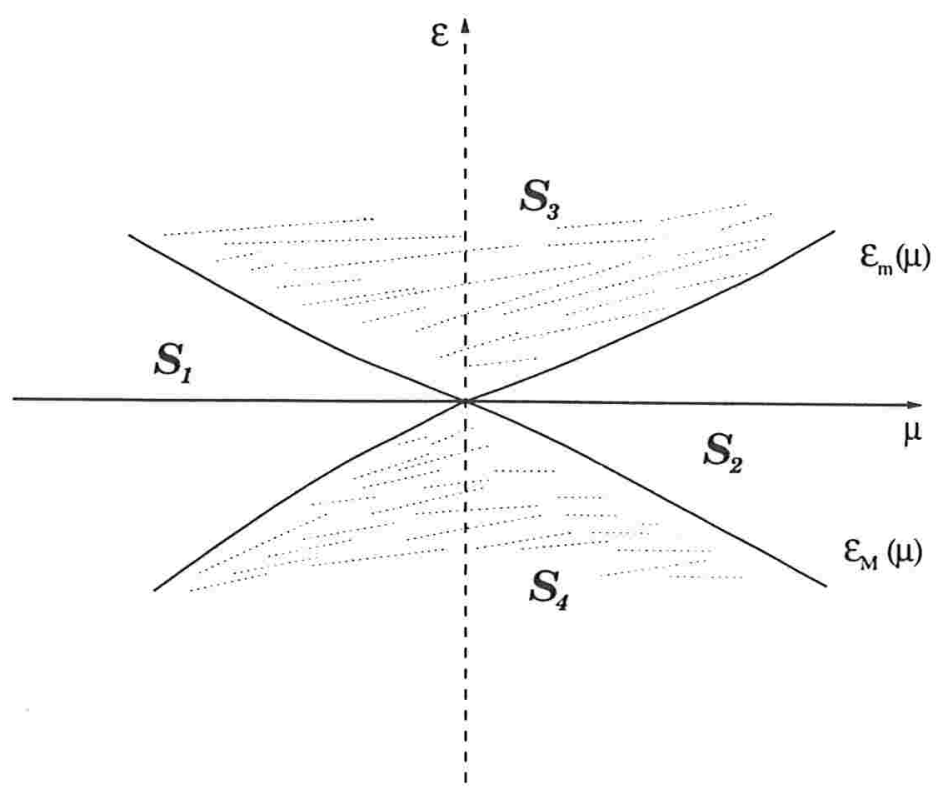

Figura 3.10: Regiōes dadas no enunciado do Teorema 3.3.1. As curvas de tangencia $\epsilon_{i}(\mu)$ estão contidas nas regiōes $S_{3}$ ou $S_{4}$, dependendo do sinal de $M\left(\phi_{i}\right)$. As curvas "especiais" $\epsilon_{m}(\mu)$ e $\epsilon_{M}(\mu)$ representam as "primeiras tangencias" entre as variedades $W_{\mu, \epsilon}^{s}\left(\theta_{1}(s)\right)$ e $W_{\mu, \epsilon}^{u}\left(\theta_{2}(s)\right)$.

onde

$$
S=-\int_{t_{-}}^{t_{+}} e^{-\int_{0}^{t} \dot{\sigma}(\tau) d \tau}\left(1+\varphi_{2}(t)^{2}\right)^{-2} Q\left(1, \varphi_{2}(t)\right) d t .
$$

Analogamente ao que se faz na prova do Teorema 3.1.2, mostra-se que para $\mu>0$ e $\epsilon_{m}(\mu)<\epsilon<\epsilon_{M}(\mu)$ (ou seja, na região $S_{2}$ ), as variedades invariantes, $W_{\mu, \epsilon}^{u}\left(\theta_{2}(s)\right.$ ) e $W_{\mu, \epsilon}^{s}\left(\theta_{1}(s)\right)$ não se intersectam: por outro lado, para $\mu<0$ e $\epsilon_{M}(\mu)<\epsilon<\epsilon_{m}(\mu)$ (região $S_{1}$ ), tem-se a mesma conclusão. Assim, as curvas $\epsilon_{m}(\mu)$ e $\epsilon_{M}(\mu)$ determinam os "primeiros pontos de tangência" entre $W_{\mu, \epsilon}^{u}\left(\theta_{2}(s)\right)$ e $W_{\mu, \epsilon}^{s}\left(\theta_{1}(s)\right)$.

2) Considerando-se a hipótese

$$
M\left(\phi_{i}\right) \neq M\left(\phi_{j}\right)
$$

para $i \neq j$, tem-se $M\left(\phi_{\min }\right)<M\left(\phi_{i}\right)<M\left(\phi_{\max }\right)$. para todo $i=1,2, \cdots, k$. com $i \neq m$ e $i \neq M$. Dessa desigualdade e do fato de que $S<0$, segue que as curvas $\epsilon_{i}(\mu)$. 
dadas por

$$
\epsilon_{i}(\mu)=-\frac{S}{M\left(\phi_{i}\right)} \mu+O\left(\mu^{2}\right)
$$

estão contidas nas regiões $S_{3}$ ou $S_{4}$, dependendo do sinal de $M\left(\phi_{i}\right)$, que determina o coeficiente angular da reta tangente à curva na origem: positivo se $M\left(\phi_{i}\right)>0$ e negativo se $M\left(\phi_{i}\right)<0$. Prova-se assim o ítem ii).

3) O ítem iii) é provado de maneira inteiramente análoga à prova do Teorema 3.1.2, observando-se o fato que, se $(\mu, \epsilon) \in S_{3}$ ou $(\mu, \epsilon) \in S_{4}$, mas $(\mu, \epsilon) \notin \epsilon_{i}(\mu)$, então $(\mu, \epsilon)$ pertence a alguma curva cuja reta tangente na origem tem coeficiente angular $S / M\left(\phi_{0}\right)$, com $\phi_{0} \neq \phi_{i}, \forall i$, donde segue que $M^{\prime}\left(\phi_{0}\right) \neq 0$. O resultado segue então aplicando-se o Teorema 2.4.2.

\subsection{Caso 2: Campos polinomiais de grau $n$ com uma reta invariante}

Os resultados da seção anterior podem ser extendidos sem esforço adicional para campos polinomiais de grau $n$, com uma reta invariante, que não contém pontos de equilíbrio, conectando dois pontos de sela hiperbólicos no infinito. Considerando novamente, sem perda de generalidade, que tal reta é dada pelo eixo-y, pode-se escrever a equação diferencial associada ao campo da seguinte forma:

$$
\begin{aligned}
& \dot{x}=x P(x, y) \\
& \dot{y}=Q(x, y),
\end{aligned}
$$

onde $P(x, y)$ e $Q(x, y)$ são polinômios de grau $n-1$ e $n$, respectivamente, $\operatorname{com} Q(0, y)>$ $0, \forall y$.

Observação: Das considerações acima segue que o grau do polinômio $Q(0, y)$ deve ser par, pois caso contrário $Q(0, y)$ teria pelo menos uma raiz real, e o campo considerado 
um equilíbrio sobre o eixo- $y$.

Perturbações periódicas do tipo

$$
\begin{aligned}
& \dot{x}=x P(x, y)+\epsilon f(\tilde{\omega} t) \\
& \dot{y}=Q(x, y)+\epsilon g(\tilde{\omega} t)
\end{aligned}
$$

$\operatorname{com} f(\tilde{\omega} t)$ satisfazendo as hipóteses dos teoremas anteriores levam às mesmas conclusòes obtidas, sendo $\tilde{\omega}=\omega\left(\sqrt{x^{2}+y^{2}}\right)^{n-1}$, com $\omega>0$ constante.

\subsection{Caso 3: Campos polinomiais com uma curva qualquer invariante}

Seja $X=(P(x, y), Q(x, y))$ um campo vetorial polinomial de grau $n$. Suponha que o campo compactificado $p\left(X^{\prime}\right)$ possui dois pontos de sela hiperbólicos, $\theta_{1}$ e $\theta_{2}$, no bordo do disco de Poincaré, conectados por uma trajetória heteroclínica $\gamma(s)$, e que o arco $\alpha(s)$ que conecta tais pontos no bordo do disco não possui singularidades (conforme Figura 3.11).

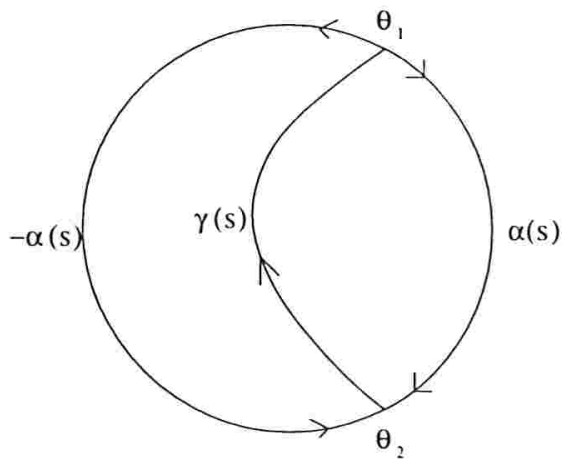

Figura 3.11: Plano de fase do campo $X$ no disco de Poincaré.

Considerando-se uma rotação de ângulo $\lambda . \operatorname{com} \lambda$ suficientemente pequeno, do cam- 
po $X$, obtem-se o sistema

$$
\begin{aligned}
& \dot{x}=\cos \lambda P(x, y)-\operatorname{sen} \lambda Q(x, y) \\
& \dot{y}=\operatorname{sen} \lambda P(x, y)+\cos \lambda Q(x, y) .
\end{aligned}
$$

Para $\lambda$ suficientemente pequeno, $\cos \lambda>0$. Assim pode-se dividir o sistema acima por $\cos \lambda$, obtendo-se o sistema equivalente

$$
\begin{aligned}
& \dot{x}=P(x, y)-\mu Q(x, y) \\
& \dot{y}=Q(x, y)+\mu P(x, y),
\end{aligned}
$$

onde $\mu=\operatorname{tg} \lambda$.

Em [25] foram estudadas perturbações autônomas de ciclos singulares infinitos nãosimétricos do tipo acima, e foi provado, entre outros, o seguinte resultado

Proposição 3.5.1 Se o ciclo singular infinito (não-simétrico) $\gamma(s) \cup \theta_{1} \cup \alpha(s) \cup \theta_{2}$ é hiperbólico, o sistema (3.22)-(3.23) possui, para $\mu>0$ suficientemente pequeno, um único ciclo limite hiperbólico, cuja imagem, via projeção central, está contida numa vizinhança do ciclo singular infinito $\gamma(s) \cup \theta_{1} \cup \alpha(s) \cup \theta_{2}$, existente para $\mu=0$. Por outro lado, para $\mu<0$ suficientemente pequeno, o sistema não possui órbitas periódicas na vizinhança de $\Gamma$ (Figura 3.12).

Observe que, neste caso, a rotação do sistema faz o papel do parâmetro $\mu$ considerado nas seções anteriores, ou seja, separa as variedades estável e instável dos pontos críticos no infinito do campo $X$. Neste caso, porém, a perturbação possui o mesmo grau do campo original, sendo ilimitada se considerarmos $(x, y) \in \mathbb{R}^{2}$. Isto constitui outra diferença entre nosso trabalho e os métodos de perturbação comumente encontrados na literatura, que consideram, em geral, perturbações por funções limitadas. O problema da não-limitação da perturabação é resolvido aqui com o artifício da compactificação. 

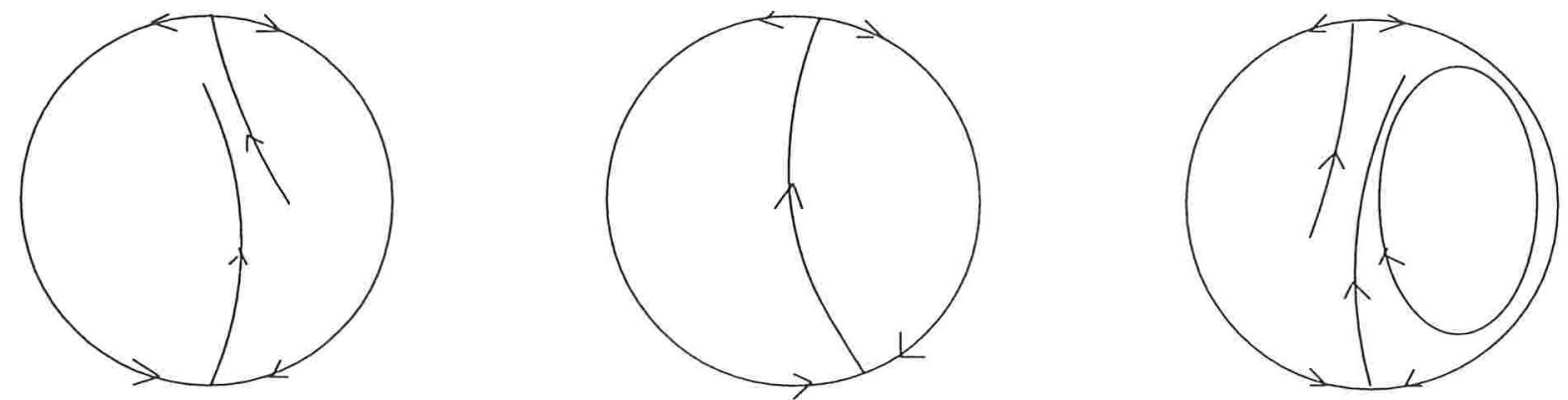

Figura 3.12: Bifurcação do sistema (3.22)-(3.23), para $\mu$ variando numa vizinhança de zero (conforme [25]).

Considere agora a seguinte perturbação periódica do campo (3.22)-(3.23)

$$
\begin{aligned}
& \dot{x}=P(x, y)-\mu Q(x, y)+\epsilon f(\tilde{\omega} t) \\
& \dot{y}=Q(x, y)+\mu P(x, y)+\epsilon g(\tilde{\omega} t) .
\end{aligned}
$$

onde $\mu$ e $\epsilon$ são parâmetros reais, $f$ e $g$ são funções de classe $C^{k} . k \geq 2$, em $\mathbb{R}$, periódicas de período $2 \pi / \tilde{\omega}$, sendo $\tilde{\omega}=\omega\left(\sqrt{x^{2}+y^{2}}\right)^{n-1}$.

Teorema 3.5.2 Nas condiçôes acima, para $\mu$ e $\epsilon$ suficientemente pequenos, o sistema (3.24)-(3.25) possui duas órbitas periódicas hiperbólicas. $\theta_{1}(s) \in \theta_{2}(s)$ no infinito, com variedades invariantes $W_{\mu, \epsilon}^{s}\left(\theta_{1}(s)\right) \in W_{\mu, \epsilon}^{u}\left(\theta_{2}(s)\right)$. Considere a função de SotomayorMelnitiov associada ao sistema, dada por

$$
M\left(\phi_{0}\right)=\int_{t_{-}}^{t_{+}} e^{-\int_{0}^{t} \tilde{\sigma}(s) d s}\left(\varphi_{1}(t)^{2}+\varphi_{2}(t)^{2}\right)^{-\frac{(n+2)}{2}}\left[P(\varphi(t)) g\left(t+o_{0}\right)-Q(\varphi(t)) f\left(t+\phi_{0}\right)\right] d t .
$$

onde $\varphi(t)$ é a solução ilimitada, contida na curva incrariante que conecta os pontos de sela no infinito. Se $M\left(\phi_{0}\right)$ possuir somente dois pontos críticos. um de máximo e um de mínimo, não-degenerados, então para $(\mu, \epsilon)$ numa vizinhança da origem, o sistema (3.24)-(3.25) apresenta diagrama de bifurcaçào equicalente ao do sitema (3.9)-(3.10). descrito no Teorema 3.1.2 (Figura 3.2). 
Prova: A demonstração é feita de maneira análoga à do Teorema 3.1.2, com pequenas adaptações, que descrevemos a seguir. Das hipóteses sobre o campo (3.24)-(3.25), segue que, para $\mu=\epsilon=0$, sua compactificação apresenta, no espaço de fase estendido $\mathrm{D}^{2} \times \mathrm{S}^{1}$, duas órbitas periódicas, dadas por

$$
\begin{aligned}
& \theta_{1}(s)=\left\{\left(\theta_{1}, 0 . \omega s+\phi_{0}\right) \mid s \in \mathbb{R} \text { e } \phi_{0} \in[0,2 \pi)\right\}, \\
& \theta_{2}(s)=\left\{\left(\theta_{2}, 0 . \omega s+\phi_{0}\right) \mid s \in \mathbb{R} \text { e } \phi_{0} \in[0,2 \pi)\right\},
\end{aligned}
$$

com variedades invariantes $W^{s}\left(\theta_{1}(s)\right)$, estável, e $W^{u}\left(\theta_{2}(s)\right)$, instável, contidas no interior de $\mathrm{D}^{2} \times \mathrm{S}^{1}$, coincidentes. Na notação do Teorema 3.1.2, a expansão em Série de Taylor em torno de $(\mu, \epsilon)=(0,0)$ da distância entre tais variedades, em uma seção $\Sigma^{\circ}$ é dada por

$$
d\left(\phi_{0}, \mu, \epsilon\right)=\frac{S \mu}{N}+\frac{M\left(\phi_{0}\right)}{N} \epsilon+R\left(\phi_{0}, \mu, \epsilon\right)
$$

onde

$$
R\left(\phi_{0}, \mu, \epsilon\right)=a\left(\phi_{0}, \mu, \epsilon\right) \mu^{2}+b\left(\phi_{0}, \mu, \epsilon\right) \mu \epsilon+c\left(\phi_{0}, \mu, \epsilon\right) \epsilon^{2},
$$

sendo $a, b, c$ funções diferenciáveis tais que $a\left(\phi_{0}, 0,0\right)=b\left(\phi_{0}, 0,0\right)=c\left(\phi_{0}, 0,0\right)=0$.

Chamando $\eta=\epsilon / \mu$ e colocando-se $\mu$ em evidência na expressão acima tem-se $d\left(\phi_{0}, \mu, \eta\right)=\frac{\mu}{N}\left(S+M\left(\phi_{0}\right) \eta+a(\mu, \mu \eta) \mu+b(\mu, \mu \eta) \mu \eta+c(\mu, \mu \eta) \eta^{2} \mu\right):=\mu \Delta\left(o_{0}, \mu, \eta\right)$. donde segue que, para $\mu \neq 0$ suficientemente pequeno, os zeros da função $\triangle$ serào também zeros da função $d$.

Considerando agora a perturbação autônoma dada pela rotação do campo, tem-se. usando a expressão (2.16) do Capítulo 2,

$$
S=-\int_{t_{-}}^{t_{+}} e^{-\int_{0}^{t} \tilde{\sigma}(s) d s}\left(\varphi_{1}(t)^{2}+\varphi_{2}(t)^{2}\right)^{-\frac{n+2}{2}}\left[P^{2}\left(\varphi_{1}(t), \varphi_{2}(t)\right)+Q^{2}\left(\varphi_{1}(t), \varphi_{2}(t)\right] d t<0\right.
$$

onde $\varphi(t)=\left(\varphi_{1}(t), \varphi_{2}(t)\right)$ é a solução do campo $\mathrm{X}$ que conecta os pontos de sela no infinito. É claro que $S<0$, já que o integrando é produto de termos positivos. 
Além disso, usando novamente a expressão (2.16), encontramos $\left.M\left(\phi_{0}\right)=\int_{t_{-}}^{t_{+}} e^{-\int_{0}^{t} \tilde{\sigma}(\tau) d \tau}\left(\varphi_{1}(t)^{2}+\varphi_{2}(t)^{2}\right)^{-\frac{n+2}{2}}\left[P(\varphi(t)) g\left(t+\phi_{0}\right)\right)-Q(\varphi(t)) f\left(t+\phi_{0}\right)\right] d t$ que é diferenciável no intervalo $\phi_{0} \in[0,2 \pi)$, e, por hipótese assume valores máximo e mínimo em pontos $\phi_{\max }$ e $\phi_{\min }$, que são não-degenerados, isto é, $M^{\prime \prime}\left(\phi_{\min }\right)>0$ e $M^{\prime \prime}\left(\phi_{\max }\right)<0$. Suponhamos, sem perda de generalidade, que

$$
M\left(\phi_{\min }\right)<0<M\left(\phi_{\max }\right)
$$

Tomando-se $\bar{\phi}_{0}$ tal que $M\left(\bar{\phi}_{0}\right) \neq 0$ e

$$
\bar{\eta}=-\frac{S}{M\left(\bar{\phi}_{0}\right)}
$$

tem-se:

$$
\begin{aligned}
& \text { i) } \Delta\left(\bar{\eta}, \bar{\phi}_{0}, 0\right)=0 \text {; } \\
& \text { ii) } \frac{\partial \Delta}{\partial \phi_{0}}\left(\bar{\eta}, \bar{\phi}_{0}, 0\right)=\bar{\eta} M^{\prime}\left(\bar{\phi}_{0}\right) \\
& \text { iii) } \frac{\partial \Delta}{\partial \eta}\left(\bar{\eta}, \bar{\phi}_{0}, 0\right)=M\left(\bar{\phi}_{0}\right)>0 .
\end{aligned}
$$

De (i)+(iii) segue. do Teorema da Função Implícita, que existe uma função $\eta=$ $\eta\left(\phi_{0}, \mu\right)$, para $\left(\phi_{0}, \mu\right) \in V i z\left(\bar{\phi}_{0}\right) \times V i z(0)$, com $\eta\left(\bar{\phi}_{0} .0\right)=\bar{\eta}=-S / M\left(\bar{\phi}_{0}\right)$, tal que

$$
\Delta\left(\eta\left(\phi_{0}, \mu\right), \phi_{0}, \mu\right)=0
$$

donde segue que $d\left(\phi_{0}, \mu, \eta\left(\phi_{0}, \mu\right)\right)=0$, para $\mu$ suficientemente pequeno. Além disso, de (ii) tem-se, dois casos a considerar:

a) $\bar{\phi}_{0}$ é ponto de máximo ou ponto de mínimo de $M$. Neste caso $\frac{\partial \Delta}{\partial \phi_{0}}\left(\bar{\eta}, \bar{\phi}_{0}, 0\right)=0$. Calculando a segunda derivada com relação a $\phi_{0}$ obtem-se

$$
\frac{\partial^{2} \Delta}{\partial \phi_{0}^{2}}\left(\bar{\eta}, \bar{\phi}_{0}, 0\right)=S<0
$$


Logo, pelo Teorema 2.1.3, $W^{s}\left(\theta_{1}(s)\right)$ e $W^{u}\left(\theta_{2}(s)\right)$ se tangenciam quadraticamente no ponto $\left(\gamma(0)+O(\mu), \bar{\phi}_{0}\right)$ para $\eta=\bar{\eta}+O(\mu)$.

b) $\bar{\phi}_{0}$ não é ponto de máximo ou de mínimo de $M$, isto é,

$$
M\left(\phi_{\min }\right)<M\left(\bar{\phi}_{0}\right)<M\left(\phi_{\max }\right), \quad \operatorname{com} M\left(\bar{\phi}_{0}\right) \neq 0 .
$$

Neste caso $\frac{\partial \Delta}{\partial \phi_{0}}\left(\bar{\eta}, \bar{\phi}_{0}, 0\right) \neq 0$ e, pelo Teorema $2.1 .2, W^{s}\left(\theta_{1}(s)\right)$ e $W^{u}\left(\theta_{2}(s)\right)$ se intersectam transversalmente no ponto $\left(\gamma(0)+O(\mu), \bar{\phi}_{0}\right)$ e, por (iii), tal intersecção se mantém para $\eta=\bar{\eta}+O(\mu)$.

Voltando-se a mudança de variáveis $\eta=\epsilon / \mu$ obtem-se as curvas

$$
\epsilon_{M}(\mu)=-\frac{S}{M\left(\phi_{\max }\right)} \mu+O\left(\mu^{2}\right)
$$

e

$$
\epsilon_{m}(\mu)=-\frac{S}{M\left(\phi_{\min }\right)} \mu+O\left(\mu^{2}\right)
$$

no plano- $(\mu, \epsilon)$, para as quais $W^{s}\left(\theta_{1}(s)\right)$ e $W^{u}\left(\theta_{2}(s)\right)$ se tangenciam quadraticamente, e fica assim provado o ítem (a) do teorema

Procedendo de maneirea análoga ao que se faz na prova do Teorema 3.1.2 mostra-se que, para $\mu>0$ suficientemente pequeno, se $\epsilon_{m}(\mu)<\epsilon<\epsilon_{M}(\mu)$, então, $W^{s}\left(\theta_{1}(s)\right) \cap$ $W^{u}\left(\theta_{2}(s)\right)=\emptyset$. No caso em que $\mu<0$, o mesmo resultado é válido, invertendo-se a desigualdade na expressão acima.

Note que os resultados desta seção dão um método para se obter tangências heteroclínicas (ou homoclínicas) e pontos heteroclínicos (homoclínicos) transversais, a partir de conexões de selas formando um ciclo singular infinito. Basta fazer uma rotação do campo para separar as variedades estável e intável das selas e em seguida considerar uma perturbação periódica da forma acima. Assim, variando-se a amplitude da perturbação periódica, obtem-se o primeiro ponto de tangência e também as intersecçòes 
transversais entre as variedades das órbitas periódicas no infinito. Além disso, o método é válido tanto para conexões de selas no infinito como para conexões de selas finitas, contidas em regiões compactas do plano.

O resultado obtido acima se baseia no fato de que as rotações são perturbaçòes transversais à variedade $\Sigma^{1}=$ CConjunto dos campos polinomiais do plano que apresentam conexões de selas no infinito\}, isto é, as rotaçòes quebram as conexões de selas. $\mathrm{O}$ resultado continua válido também para outras perturbações mais gerais, desde que satisfaçam essa condição de transversalidade (representada analíticamente por $S \neq 0$ nos teoremas deste capítulo). Utilizamos as rotações no estudo acima, com objetivo de estabelecer um algoritmo para obtenção de tangências e transversalidade entre variedades invariantes dos ciclos singulares infinitos, o que pode ser usado também para análise de conexões homoclínicas em regiões compactas do plano.

Observação 3.5.3 Campos quadráticos com uma parábola e também com uma hipérbole invariante foram considerados em [9]. Foi provado que um campo quadrático que possui uma hipérbole invariante não possui ciclos-limites. Porém, a teoria aqui apresentada não pode ser aplicada neste caso, cuja forma normal do campo é dada por

$$
\begin{aligned}
& \dot{x}=a(x y-c)+y(a x+b y+c) \\
& \dot{y}=\beta(x y-y)+x(a x+b y+c) .
\end{aligned}
$$

pois os pontos de equilibrio no infinito, conectados pela hipérbole, são degenerados (todos os autovalores do Jacobiano do campo compactificado, calculado nestes pontos. sào

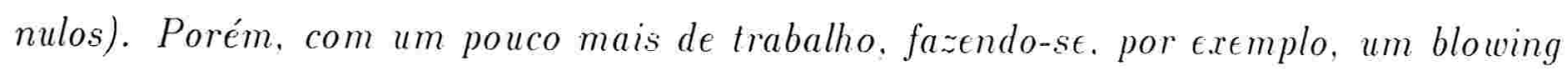
up das singularidades degeneradas, pode-se analisar tal problema.

\subsection{Caso 4: Ciclos singulares semi-infinitos}

A teoria desenvolvida nas seções anteriores se aplica também a campos polinomiais com um ciclo singular semi-infinito. que definimos como sendo um ciclo formado pela 
conexão de uma sela hiperbólica, p, na parte compacta do plano com duas selas, $\theta_{1}$ e $\theta_{2}$, no infinito (Figura $3.13(\mathrm{a})$ ).

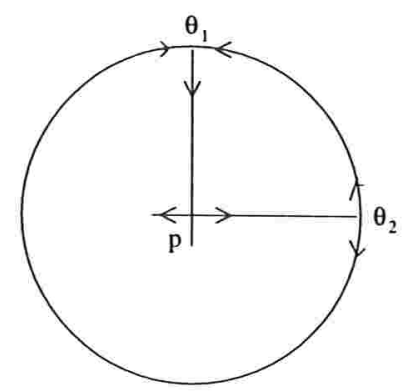

(a)

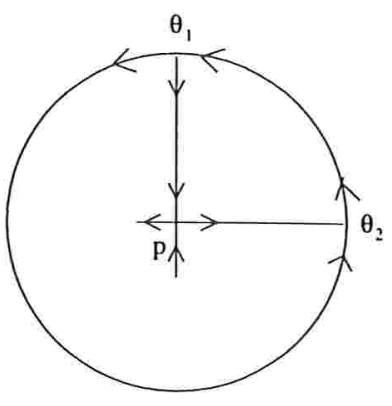

(b)

Figura 3.13: Ciclos singulares semi-infinitos.

Neste caso, dependendo da perturbação periódica considerada, pode haver a quebra de uma ou das duas conexões da sela finita com as selas no infinito, que pode levar a existência de tangências ou intersecção transversal das variedades invariantes. Por exemplo, se tivermos retas invariantes vertical e horizontal, conectando $p \operatorname{com} \theta_{1}$ e com $\theta_{2}$, respectivamente, pode-se fazer uma perturbação de maneira que somente uma das conexões se quebre.

De qualquer forma, usando-se as técnicas apresentadas na seção anterior, podese calcular uma primeira aproximação da Função de Separação entre as variedades invariantes dos pontos de equilíbrio e detectar as tangências e intersecções transversais entre tais variedades.

Mais uma vez, neste caso também é necessário um estudo global do plano de fase do sistema, via compactificação, para se estudar tais fenômenos.

Um caso particular de ciclo semi-infinito é aquele em que os pontos críticos no infinito são do tipo sela-nó, com variedade forte transversal e variedade central tangente ao equador (chamado sela-nó tangencial) (Figura 3.13 (b)). O desdobramento genérico de ciclos singulares contendo pontos de sela-nó tangencias no infinito também foram 
estudados em [25], onde foi provado, sob certas condições, o surgimento de ciclos-limites de grande amplitude, com o desdobramento do pontos de sela-nó no infinito.

Pode-se também considerar perturbações periódicas dos ciclos mostrados na Figura 3.13 (b).

Primeiramente, observe que, se considerarmos perturbaçòes periódicas que dependem apenas do tempo, e não das variáveis espaciais, tais perturbações não desdobram o equilíbrio tipo sela-nó no infinito, já que sua existência é dada pela equação $A_{n}(\theta)=0$ e seu tipo é determinado apenas pelos termos de grau $n$ e $n-1$ do polinômio, conforme expressão da matriz jacobiana do campo compactificado, que é dada por

$$
\left(\begin{array}{cc}
A_{n}^{\prime}(\theta) & A_{n-1}(\theta) \\
0 & -R_{n}(\theta)
\end{array}\right) .
$$

Logo, mesmo mediante a perturbação periódica (de grau zero) do campo, o ponto de sela-nó e, consequentemente, sua variedade forte. continua existindo (isto é, tal perturbação não desdobra a sela-nó). Pode-se entào considerar a análise da quebra da conexão da sela-nó no infinito com a sela hiperbólica na parte compacta do plano, com relação à existência de tangências e intersecções transversais. Toda a teoria apresentada nas seçòes anteriores se aplica também neste caso.

Observação 3.6.1 Um sistema polinomial bastante conhecido. que aprestnta conexò de um ponto de sela hiperbólico na parte compacta do plano (de fato, na origem). com pontos do tipo sela-nó tangencial no infinito. é o modelo clássico de Predador-Presa. com as equações de Lotka-Volterra. 


\section{Capítulo 4}

\section{Órbitas Periódicas Ressonantes e Bifurcações Subharmônicas}

Os ciclos singulares infinitos e semi-infinitos de campos polinomiais, considerados no capítulo anterior, podem ser de dois tipos: atratores (ou repulsores), ou acumulados por órbitas periódicas (Figura 4.1).

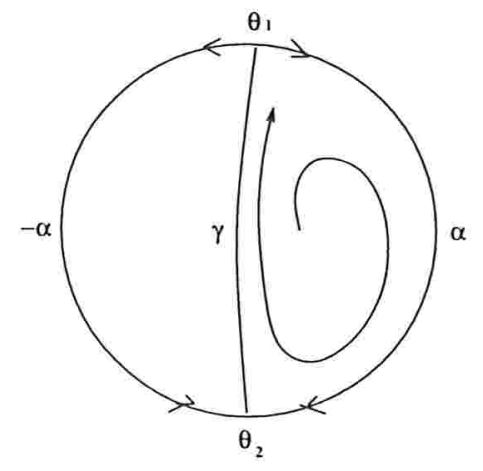

(a)

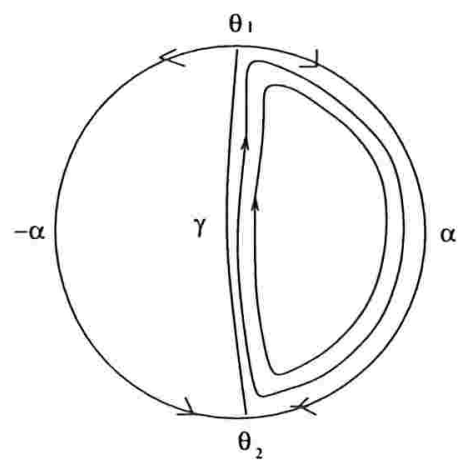

(b)

Figura 4.1: Ciclos singulares infinitos: a) atrator; b) acumulado por órbitas periódicas.

No capítulo anterior foram analisadas as bifurcações heteroclínicas dos ciclos singulares infinitos, decorrentes de perturbações periódicas do campo polinomial. 
Neste capítulo estuda-se o efeito das perturbações periódicas sobre as órbitas periódicas que se acumulam nos ciclos singulares infinitos. Analisa-se também a interação existente entre as bifurcações heteroclínicas de tais ciclos (tangências e intersecções transversais), estudadas no Capítulo 3, e as bifurcações que ocorrem com o anel de órbitas periódicas que se acumula neles.

Os resultados obtidos dão informações sobre a existência de órbitas periódicas do sistema perturbado, para determinados valores dos parâmetros. e estào no contexto dos trabalhos [8] e [16], porém com diferenças significativas: os anéis de órbitas periódicas aqui considerados estão próximos do infinito e se acumulam em ciclos singulares infinitos, enquanto que nos trabalhos citados considera-se somente regiōes compactas do plano de fase. Além disso, os campos vetoriais e as perturbaçòes periódicas que consideramos são distintos daqueles considerados em [8] e [16]. Entretanto, as condições encontradas para existência de órbitas periódicas do sistema perturbado são semelhantes àquelas apresentadas nos referidos trabalho.

As técnicas aqui utilizadas têm como base a Proposição 4.1.1, da seção seguinte, sobre a solução da equação variacional associada a uma equaçào diferencial ordinária, na vizinhança de uma órbita regular.

\subsection{Definições e resultados preliminares}

Com objetivo de formular os resultados que se pretende obter neste capítulo e estabelecer algumas definiçòes e resultados preliminares que serào utilizados, considere o sistema

$$
\dot{u}=f(u)+\epsilon g(u, t),
$$

onde $u \in \mathbb{R}^{2}, \epsilon$ é um parâmetro real e $g$ uma funçào de classe $C^{k}, k \geq 2$, em $\mathbb{R}$, periódica de período $T$, em $t$.

Suponha que o sistema acima possui, para $\epsilon=0 . \mathrm{m}$ anel de órbitas periódicas, contendo uma órbita periódica $\Gamma$. cujo período está $\mathrm{\epsilon m}$ ressonância com o período $T$ 
da função $g$, isto é, existe um número natural $m$ tal que o período mínimo de $\Gamma$ é igual a $m T$. Neste caso, diz-se que $\Gamma$ é uma órbita periódica ressonante. Nestas condições, o problema que se coloca é o de determinar se existem valores de $\epsilon$ diferentes de zero, para os quais o sistema perturbado (4.1) possui órbitas periódicas de período $m T$, próximas de $\Gamma$. Tais órbitas são chamadas subharmônicas de ordem $m$, pois seu período é igual a $m$ vezes o período da função de perturbação $g(u, t)$. Neste contexto, procura-se determinar curvas $\epsilon \rightarrow \sigma(\epsilon)$ no plano de fase do sistema, com $\sigma(0) \in \Gamma$, tais que $\sigma(\epsilon)$ é valor inicial de uma órbita subharmônica de ordem $m$ do sistema perturbado. Em outras palavras, procura-se subharmônicas que bifurcam da órbita ressonante $\Gamma$, sendo $\sigma(\epsilon)$ uma curva de bifurcação de subharmônicas.

A idéia que usaremos para resolução do problema acima é, tendo em conta o caráter periódico de $g$, considerar (4.1) como um sistema autônomo definido no cilindro $\mathbb{R}^{2} \times \mathrm{S}^{1}$, através da mudança de variáveis $\phi=t, \dot{\phi}=1$, com o que tem-se

$$
\begin{aligned}
& \dot{u}=f(u)+\epsilon g(u, \phi) \\
& \dot{\phi}=1,
\end{aligned}
$$

com $u \in \mathbb{R}^{2}$ e $\phi \in \mathrm{S}^{1}$, onde $\mathrm{S}^{1}$ é identificado com o intervalo $[0, T)$, através da consideração de $[0, T)$ como o espaço quociente $\mathbb{R} / \mathrm{TZ}$.

Assim, as órbitas periódicas do sistema não-perturbado ((4.2)-(4.3), com $\epsilon=0$ ) passam a ser toros invariantes, contidos em $\mathbb{I R}^{2} \times \mathrm{S}^{1}$ (Figura 4.2).

Dessa forma, pode-se tomar uma seção transversal ao fluxo do sistema (4.2)-(4.3) (Figura 4.2), da forma

$$
\Sigma^{\phi_{0}}=\left\{(u, \phi) \mid u \in \mathbb{R}^{2} \text { e } \phi=\phi_{0} \in[0, \mathrm{~T})\right\}
$$

sobre a qual define-se a m-ésima iterada da transformação de Poincaré, $P_{\epsilon}^{m}: \Sigma^{\phi_{0}} \longrightarrow$ $\Sigma^{\phi_{0}}$, dada por

$$
P_{\epsilon}^{m}(\xi)=u(m T, \xi, \epsilon),
$$



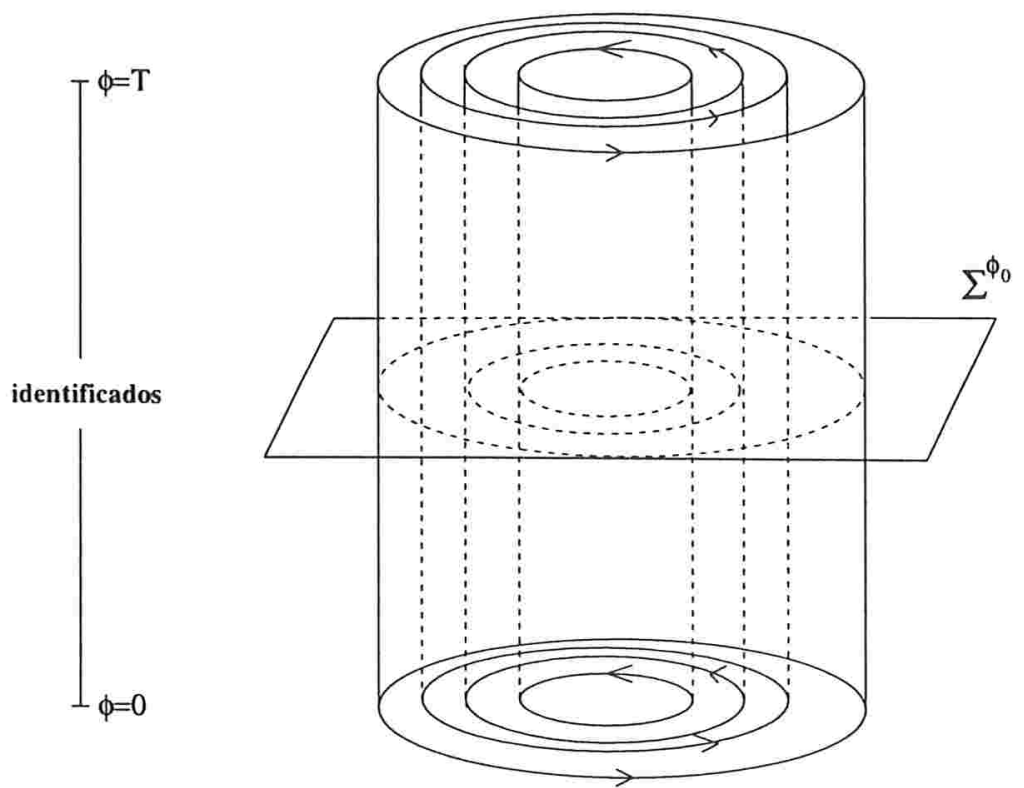

Figura 4.2: As órbitas periódicas do anel do sistema (4.1), com $\epsilon=0$, passam a ser toros invariantes para o sistema (4.2)-(4.3).

onde $u(t, \xi, \epsilon)$ é a solução de (4.2)-(4.3) com $u(0, \xi, \epsilon)=\xi \in \Sigma^{\phi_{0}}$. Assim, se $\Gamma$ é uma órbita periódica ressonante de (4.1), então, para $\epsilon=0$ todo ponto $\xi \in \Gamma$ é ponto fixo de $P_{0}^{m}(\xi)$. De fato,

$$
P_{0}^{m}(\xi)=u(m T, \xi, 0)=\xi
$$

Tais pontos fixos correspondem a órbitas periódicas de período $m$ do sistema (4.2)-(4.3). $\operatorname{com} \epsilon=0$, que sào subharmônicas de ordem $m$.

Para verificar se algum desses pontos fixos (e, consequentemente, dessas órbitas periódicas de período $m$ ) persiste para $\epsilon \neq 0$ suficientemente pequeno, considera-se então a função

$$
\delta(\xi, \epsilon)=P_{\epsilon}^{m}(\xi)-\xi=u(m T, \xi, \epsilon)-\xi
$$

Assim, é claro que os zeros da função $\delta$, que dão os pontos fixos de $P_{\epsilon}^{m}$, correspondem a órbitas periódicas de período $m T$ do sistema perturbado (4.2)-(4.3), que são 
subharmônicas de ordem $m$, desde que $P_{\epsilon}^{j}(\xi) \neq \xi$, para $1<j<m$.

Para estudar a existência de zeros da função $\delta$ definida acima, usamos, conforme feito em [5], dois resultados clássicos da Teoria Qualitativa das Equações Diferenciais Ordinárias, que serão enunciados na próxima sub-seção (Proposição 4.1.1 e Lema 4.1.4). Omitem-se as provas, que podem ser encontradas em [4] ou [5]. Com base nestes resultados, provamos um teorema fundamental (Teorema 4.1.6) que será usado no estudo das bifurcações subharmônicas que ocorrem no anel de órbitas periódicas que se acumula no ciclo singular infinito de um campo polinomial no plano.

\subsubsection{Equações variacionais e bifurcações subharmônicas}

A solução da primeira equação variacional

$$
\dot{W}=D f\left(\varphi_{t}(\xi)\right) W
$$

associada a um sistema da forma $\dot{u}=f(u)$, em torno de uma solução $u=\varphi_{t}(\xi)$, pode ser obtida com a seguinte proposição, conforme [13]. Para enunciá-la, lembremos antes que, o divergente e o rotacional de uma função vetorial $f: \mathbb{R}^{2} \rightarrow \mathbb{R}^{2}$, com $f(x, y)=\left(f_{1}(x, y), f_{2}(x, y)\right)$, são definidos por:

$$
\begin{aligned}
& \operatorname{div} f(x, y):=\frac{\partial f_{1}}{\partial x}(x, y)+\frac{\partial f_{2}}{\partial y}(x, y), \\
& \operatorname{rot} f(x, y):=\frac{\partial f_{2}}{\partial x}(x, y)-\frac{\partial f_{1}}{\partial y}(x, y),
\end{aligned}
$$

e que a curvatura escalar de uma curva diferenciável $t \rightarrow(x(t), y(t))$ é dada por

$$
\kappa:=\frac{\dot{x} \ddot{y}-\dot{y} \ddot{x}}{\left(\dot{x}^{2}+\dot{y}^{2}\right)^{3 / 2}} .
$$

Proposição 4.1.1 Seja $\varphi_{t}$ o fluxo da equação diferencial $\dot{u}=f(u), u \in \mathbb{R}^{2}$. Se $f(u) \neq 0$, então a matriz fundamental $t \rightarrow \Phi(t)$, com $\Phi(0)=I d$, solução da equação variacional homogênea

$$
\dot{W}=D f\left(\varphi_{t}(\xi)\right) W
$$


associada a $\dot{u}=f(u)$, é tal que

$$
\begin{gathered}
\Phi(t) f(\xi)=f\left(\varphi_{t}(\xi)\right), \\
\Phi(t) f^{\perp}(\xi)=a(t, \xi) f\left(\varphi_{t}(\xi)\right)+b(t, \xi) f^{\perp}\left(\varphi_{t}(\xi)\right),
\end{gathered}
$$

onde

$$
\begin{gathered}
b(t, \xi)=\frac{\|f(\xi)\|^{2}}{\left\|f\left(\varphi_{t}(\xi)\right)\right\|^{2}} e^{\int_{0}^{t} \operatorname{div} f\left(\varphi_{s}(\xi)\right) d s}, \\
a(t, \xi)=\int_{0}^{t}\left(2 \kappa(s, \xi)\left\|f\left(\varphi_{s}(\xi)\right)\right\|-\operatorname{rot} f\left(\varphi_{s}(\xi)\right)\right) b(s, \xi) d s .
\end{gathered}
$$

Pelo que sabemos, a prova da Proposição acima apareceu pela primeira vez em [13], porém com um pequeníssimo erro, qual seja, a falta do escalar 2 multiplicando a curvatura $\kappa$, que comparece no integrando da funçào $a(t, \xi)$. A prova com a correção pode ser encontrada no artigo [4] ou no livro [5], pg. 331. As contas são trabalhosas. porém trata-se de simples verificação calculatória.

Observação 4.1.2 As funções a e b que aparecem no teorema acima tem um significado geométrico bastante importante: se $\xi$ pertence a uma órbita periódica $\Gamma$, de período $T$, e $\Sigma$ é uma seção transversal a $\Gamma \mathrm{em} \xi$, então

$$
b(T, \xi)=\exp \left(\int_{0}^{T} \operatorname{div} f\left(\varphi_{s}(\xi)\right) d s\right),
$$

que é exatamente a derivada da transformação de Poincaré associada a $\Gamma$, no ponto $\xi=\Gamma \cap \Sigma[5]$. Por outro lado, se $\Gamma$ está contida $\epsilon m$ um ant $\mathcal{A}$ de órbitas periódicas $\epsilon$ $\Sigma$ é uma seção transversal ortogonal a esse anel, então a função a $(T, \xi)$ é um múltiplo da derivada $T^{\prime}(\xi)$, da função periodo $T(p)$, que associa a cada ponto $p \in \mathcal{A} \cap \Sigma$ o período mínimo da órbita periódica que passa por p. conforme [4] (ou [.5]. pg.329).

Esta observação será utilizada adiante, juntamente com a definiçào a seguir. 
Definição 4.1.3 Seja $\mathcal{A}$ um anel de órbitas periódicas do sistema $\dot{u}=f(u)$, com $u \in \mathbb{R}^{2}$. Se a função período, $T(p)$, que associa a cada ponto $p \in \mathcal{A}$ o período mínimo da órbita periódica que passa por $p$, for tal que $T^{\prime}(p) \neq 0, \forall p$, então diz-se que o anel $\mathcal{A}$ é um anel regular de órbits periódicas.

No caso em que se considera uma equação da forma $\dot{u}=f(u)+\epsilon g(u, t)$, que é o caso de interesse neste trabalho, dada uma solução $\varphi_{t}(\xi, \epsilon)$, a equação variacional em torno desta solução é dada por

$$
\dot{W}=D f\left(\varphi_{t}(\xi, 0)\right) W+g\left(\varphi_{t}(\xi, 0), t\right)
$$

que é chamada de segunda equação variacional. A solução desta equação pode ser obtida utilizando-se a Proposição 4.1 .1 acima, aplicando-se o método da variação das constantes, já que sua parte homogênea coincide com a primeira equação variacional, considerada na referida proposição. Tem-se então o seguinte resultado (a prova se encontra em [4]):

Lema 4.1.4 Seja $\varphi_{t}$ o fluxo da equação diferencial $\dot{u}=f(u), u \in \mathbb{R}^{2}$. Se $f(u) \neq 0$, então a solução $t \rightarrow W(t)$, do problema de valor inicial

$$
\dot{W}=D f\left(\varphi_{t}(\xi)\right) W+g\left(\varphi_{t}(\xi), t\right), \quad W(0)=0
$$

é dada por

$$
W(t)=[N(t)+a(t, \xi) M(t)] f\left(\varphi_{t}(\xi)\right)+[b(t, \xi) M(t)] f^{\perp}\left(\varphi_{t}(\xi)\right),
$$

com

$$
\begin{gathered}
N(t):=\int_{0}^{t}\left[\frac{1}{\|f\|^{2}}<f, g>-\frac{a(s, \xi)}{b(s, \xi)\|f\|^{2}}<f^{\perp}, g>\right]\left(\varphi_{s}(\xi)\right) d s, \\
M(t):=\int_{0}^{t}\left[\frac{1}{b(s, \xi)\|f\|^{2}}<f^{\perp}, g>\right]\left(\varphi_{s}(\xi)\right) d s,
\end{gathered}
$$

onde $a$ e $b$ são as funçôes definidas na Proposição 4.1.1, e $<$, > denota o produto interno usual do $\mathbb{R}^{2}$. 
Prova: ver [4] ou [5].

Suponha agora que o sistema $\dot{u}=f(u)+\epsilon g(u, t)$ possui, para $\epsilon=0$, um ant regular de órbitas periódicas, contendo uma órbita periódica $\Gamma$, cujo período está em ressonância com o período $T$ da funçào $g$. Na próxima sub-seção mostra-se (Teorema 4.1.6) que, nestas condições, os zeros da função $\delta(\xi, \epsilon)=P_{\epsilon}^{m}(\xi)-\xi$, definida em (4.5). estão relacionados aos zeros simples da função $M(t)$, que aparece na componente da solução $W(t)$, na direção de $f^{\perp}$, no Lema 4.1.4.

\subsubsection{Bifurcações subharmônicas em um anel regular de órbitas periódicas}

Lema 4.1.5 Se $\xi$ pertence a uma órbita periódica ressonante de $\dot{u}=f(u)$, então $f(\xi)$ pertence ao $\operatorname{Ler}\left[\delta_{\xi}(\xi, 0)\right]$, onde $\delta(\xi, \epsilon)=u(m T, \xi, \epsilon)-\xi$. definida $\mathrm{em}$ (4.5).

\section{Prova:}

$$
\begin{aligned}
\delta_{\xi}(\xi, 0) f(\xi) & =\left.\frac{d}{d t}(\delta(u(t, \xi, 0), 0))\right|_{t=0} \\
& =\left.\frac{d}{d t}(u(m T, u(t, \xi, 0), 0)-u(t, \xi, 0))\right|_{t=0} \\
& =\left.\frac{d}{d t}(u(m T+t, \xi, 0)-u(t, \xi, 0))\right|_{t=0} \\
& =f(u(m T, \xi, 0))-f(\xi)=0 .
\end{aligned}
$$

Como consequência deste lema, fica claro que não se pocle aplicar diretamente o Teorema da Funçào Implícita à função $\delta(\xi, \epsilon)$ na tentativa de se encontrar seus zeros. pois, mesmo sendo $\delta(\xi, 0)=0, \delta_{\xi}(\xi, 0)$ não é um isomorfismo. Usa-se então um método de reduçảo, conhecido por Método de Lyapunov-Schmidt, para resolver o problema. Trata-se de considerar projeções de $\delta$ em espaços adequados e usar o Teorema da Função Implícita para encontrar os zeros destas projeções, conforme o seguinte teorema. 
Teorema 4.1.6 Suponha que o sistema $\dot{u}=f(u)+\epsilon g\left(u, t+\phi_{0}\right)$, definido em (4.2)(4.3), possui, para $\epsilon=0$, um anel regular $\mathcal{A}$ de órbitas periódicas, contido na seção transversal $\Sigma^{\phi_{0}}=\left\{(u, \phi) \mid u \in \mathbb{R}^{2}\right.$ e $\left.\phi=\phi_{0} \in[0, T)\right\}$ (Figura 4.2). Suponha mais, que existe uma órbita periódica $\Gamma$, contida em $\mathcal{A}$, cujo período está em ressonância com o período $T$ da função $g$, isto é, existe um número natural $m$ tal que o período mínimo de $\Gamma$ é igual a $m T$. Considere a função

$$
M\left(s, \phi_{0}\right)=\int_{0}^{m T} e^{-\int_{0}^{t} d i v f\left(\varphi_{\tau+s}(v)\right) d \tau}<f^{\perp}\left(\varphi_{t+s}(v)\right), g\left(\varphi_{t+s}(v), t+\phi_{0}\right)>d t,
$$

onde $\varphi_{t}(v)$ é uma parametrização de $\Gamma$, e a transformação de Poincaré $P_{\epsilon}^{m}: \Sigma^{\phi_{o}} \rightarrow \Sigma^{\phi_{0}}$, definida por $P_{\epsilon}^{m}(\xi)=u(m T, \xi, \epsilon)$, onde $u(t, \xi, \epsilon)$ é a solução de (4.2)-(4.3) tal que $u(0, \xi, \epsilon)=\xi \in \Sigma^{\phi_{0}}$. Se existir um ponto $\left(\bar{s}, \bar{\phi}_{0}\right)$ tal que $M\left(\bar{s}, \bar{\phi}_{0}\right)=0$ e $\frac{\partial M}{\partial \phi_{0}}\left(\bar{s}, \bar{\phi}_{0}\right) \neq 0$, então, para $\epsilon \neq 0$ suficientemente pequeno, $P_{\xi}^{m}(\xi)$ possui um ponto fixo, que bifurca de um ponto de $\Gamma$. Tal ponto fixo corresponde a uma órbita subharmônica de ordem $m$ do sistema perturbado (4.2)-(4.3).

Prova: Sejam $\varphi_{t}$ o fluxo do sistema $\dot{u}=f(u)$ e $\psi_{t}$ o fluxo do sistema ortogonal $\dot{u}=f^{\perp}(u)$. Considere um sistema (local) de coordenadas $(r, s)$, definido em uma vizinhança da órbita ressonante $\Gamma$, inteiramente contida no anel $\mathcal{A} \subset \Sigma^{\phi_{0}}$, através da seguinte função

$$
H(r, s)=\psi_{r} \varphi_{s}(v)
$$

onde $s \in[0, m T], r \in V i z(0)$ e $v \in \Gamma$, sendo um ponto fixo, mas arbitrário. Assim tem-se

$$
H_{r}(r, s)=f^{\perp}(H(r, s)) \quad e \quad H_{s}(r, s)=D \psi_{r}\left(\varphi_{s}(v)\right) f\left(\varphi_{s}(v)\right)
$$

que são linearmente independentes e, portanto, para cada $\xi$ pertencente a uma vizinhança de $\Gamma$ em $\Sigma^{\phi_{0}}$, existe $(r, s)$ tal que $\xi=H(r, s)$. Escrevendo a função $\delta(\xi, \epsilon)=$ $P_{\epsilon}^{m}(\xi)-\xi=u(m T, \xi, \epsilon)-\xi$ nas coordenadas $(r, s)$ obtem-se

$$
\Delta(r, s, \epsilon)=\delta(H(r, s), \epsilon)
$$


Assim, os zeros da função $\Delta(r, s, \epsilon)$ correspondem aos pontos fixos da transformação de Poincaré $P_{\epsilon}^{m}: \Sigma^{\phi_{0}} \rightarrow \Sigma^{\phi_{0}}$. Usemos o Teorema da Funçào Implícita para encontrá-los.

Primeiramente tem-se $\Delta(0, s, 0)=\delta\left(\varphi_{s}(v), 0\right)=u\left(m T, \varphi_{s}(v), 0\right)-\varphi_{s}(v)=0$.

Considere agora a projeção de $\Delta$ na direção de $f\left(\varphi_{s}(v)\right)$ dada por

$$
\mathcal{P}(r, s, \epsilon)=<\Delta(r, s, \epsilon), f\left(\varphi_{s}(v)\right)>
$$

A derivada parcial de $\mathcal{P}$, com relação a $r$, calculada em $(0, s, 0)$ é dada por

$$
<\Delta_{r}(0, s, 0), f\left(\varphi_{s}(v)\right)>
$$

sendo que $\Delta_{r}(0, s, 0)=\delta_{\xi}\left(\varphi_{s}(v), 0\right) f^{\perp}\left(\varphi_{s}(v)\right)$. Agora,

$$
\begin{aligned}
\delta_{\xi}\left(\varphi_{s}(v), 0\right) f^{\perp}\left(\varphi_{s}(v)\right) & =\left.\frac{\partial}{\partial r}\left(u\left(m T, \psi_{r}\left(\varphi_{s}(v)\right), 0\right)-\psi_{r}\left(\varphi_{s}(v)\right)\right)\right|_{r=0} \\
& =\left.\left(u_{\xi}\left(m T, \psi_{r}\left(\varphi_{s}(v)\right), 0\right) \dot{\psi}_{r}\left(\varphi_{s}(v)\right)-\dot{\psi}_{r}\left(\varphi_{s}(v)\right)\right)\right|_{r=0} \\
& =u_{\xi}\left(m T, \varphi_{s}(v), 0\right) f^{\perp}\left(\varphi_{s}(v)\right)-f^{\perp}\left(\varphi_{s}(v)\right),
\end{aligned}
$$

onde $u_{\xi}\left(m T, \varphi_{s}(v) .0\right)$ é a matriz fundamental da equaçào variacional $\dot{W}=D f\left(\vartheta_{s}(v)\right) W$. com $W(0)=I d$. Logo, usando a Proposição 4.1.1, tem-se

$$
\begin{array}{r}
u_{\xi}\left(m T, \varphi_{s}(v), 0\right) f^{\perp}\left(\varphi_{s}(v)\right)= \\
a\left(m T, \varphi_{s}(v)\right) f\left(\varphi_{s}(v)\right)+b\left(m T, \varphi_{s}(v)\right) f^{\perp}\left(\varphi_{s}(v)\right),
\end{array}
$$

que, substituido na expressão anterior, fornece

$$
\delta_{\xi}\left(\varphi_{s}(v), 0\right) f^{\perp}\left(\varphi_{s}(v)\right)=a\left(m T, \varphi_{s}(v)\right) f\left(\varphi_{s}(v)\right)+\left[b\left(m T, \varphi_{s}(v)\right)-1\right] f^{\perp}\left(\varphi_{s}(v)\right) .
$$

Como a órbita periódica $\Gamma$ é não-hiperbólica, tem-se $b\left(m T, \varphi_{s}\left(c^{\prime}\right)\right)=1$ (conforme observação 4.1.2). Logo. da expressão acima segue que

$$
\Delta_{r}(0, s, 0)=b(s, v) a\left(m T, \varphi_{s}(v)\right) f\left(\varphi_{s}\left(v^{\prime}\right)\right),
$$

donde tem-se

$$
\left.<\Delta_{r}(0, s, 0) \cdot f\left(\varphi_{s}(v)\right)\right\rangle=b(s \cdot v) a\left(m T . \varphi_{s}(v)\right)\left\|f\left(\hat{\varphi}_{s}\left(v^{\prime}\right)\right)\right\|^{2} .
$$


que é diferente de zero, pois $b \neq 0, f$ não se anula sobre a órbita periódica $\Gamma$ e $a\left(m T, \varphi_{s}(v)\right) \neq 0$, pois, por hipótese, $\Gamma$ está contida em um anel regular $\mathcal{A}$ de órbitas periódicas, e $a$ é um múltiplo da função período (conforme observação 4.1.2).

Assim, como $\mathcal{P}(0, s, 0)=0$ e $\mathcal{P}_{r}(0, s, 0) \neq 0$, segue do Teorema da Função Implícita que, para $\epsilon \neq 0$ suficientemente pequeno, existe uma função $h=h(s, \epsilon), \operatorname{com} h(s, 0)=0$, tal que

$$
\mathcal{P}(h(s, \epsilon), s, \epsilon)=<\Delta(h(s, \epsilon), s, \epsilon), f\left(\varphi_{s}(v)\right)>=0 .
$$

Considere agora $\tilde{\Delta}$ a projeção de $\Delta(h(s, \epsilon), s, \epsilon)$ na direção de $f^{\perp}$, isto é,

$$
\tilde{\Delta}(s, \epsilon)=<\Delta(h(s, \epsilon), s, \epsilon), f^{\perp}\left(\varphi_{s}(v)\right)>\text {. }
$$

Como $\Delta(0, s, 0)=0$ e $h(s, 0)=0$, tem-se $\tilde{\Delta}(s, 0)=0$. Então, expandindo $\tilde{\Delta}$ em Série de Taylor em $\epsilon$, em torno de $\epsilon=0$, obtem-se

$$
\tilde{\Delta}(s, \epsilon)=\epsilon\left[\tilde{\Delta}_{\epsilon}(s, 0)+O(\epsilon)\right]
$$

donde fica claro que os zeros simples da função $\tilde{\Delta}_{\epsilon}(s, 0)$ correspondem aos zeros de $\Delta(r, s, \epsilon)$, para $\epsilon \neq 0$, suficientemente pequeno.

Para finalizar a prova, resta mostrar que os zeros simples de $\tilde{\Delta}_{\epsilon}(s, 0)$ correspondem aos zeros da expressão $M\left(s, \phi_{0}\right)$ do enunciado do teorema. Para tanto, derivando $\tilde{\Delta}$ com relação a $\epsilon$, e calculando em $\epsilon=0$ obtem-se

$$
\left.\tilde{\Delta}_{\epsilon}(s, 0)=<\Delta_{r}(h(s, 0), s, 0) h_{\epsilon}(s, 0)+\Delta_{\epsilon}(h(s, 0), s, 0)\right), f^{\perp}\left(\varphi_{s}(v)\right)>.
$$

De $h(s, 0)=0$ e da expressão de $\Delta_{r}$ obtida em (4.6), tem-se que

$$
\tilde{\Delta}_{\epsilon}(s, 0)=<\Delta_{\epsilon}(0, s, 0), f^{\perp}\left(\varphi_{s}(v)\right)>\text {. }
$$

Por outro lado, da definição de $\Delta(r, s, \epsilon)$ tem-se $\Delta_{\epsilon}(r, s, 0)=u_{\epsilon}(m T, r, s, 0)$, onde $u_{\epsilon}(t, r, s, 0)$ é solução da equação variacional $\dot{W}=D f\left(\varphi_{t}(\xi)\right) W+g\left(\varphi_{t}(\xi, 0), t+\phi_{0}\right)$, que, pelo lema 4.1 .4 é igual a

$$
W(t)=[N(t)+a(t, \xi) M(t)] f\left(\varphi_{t}(\xi)\right)+[b(t, \xi) M(t)] f^{\perp}\left(\varphi_{t}(\xi)\right) .
$$


Em coordenadas $(r, s)$ tem-se $\xi=H(r, s)=\psi_{r} \varphi_{s}(v)$. Como queremos $\Delta_{\epsilon}(0, s, 0)=$ $u_{\epsilon}(m T, 0, s, 0)$, tomando $r=0$, donde $\xi=\varphi_{s}(v)$, e considerando $t=m T$ na expressào da função $M(t)$ que aparece na componente de $f^{\perp} \mathrm{em} W(t)$, dada no lema 4.1.4, obtemse

$$
M(m T)=\frac{1}{\left\|f\left(\varphi_{s}(v)\right)\right\|^{2}} \int_{0}^{m T} e^{-\int_{0}^{t} \operatorname{divf}\left(\varphi_{\tau+s}(v) d \tau\right.}<f^{\perp}\left(\varphi_{t+s}(v)\right), g\left(\varphi_{t+s}(v), t+\phi_{0}\right)>d t .
$$

Portanto,

$$
\tilde{\Delta}_{\epsilon}(s, 0)=<\Delta_{\epsilon}(0, s, 0), f^{\perp}\left(\varphi_{s}(v)\right)>=b(m T, v)\left\|f^{\perp}\left(\varphi_{s}(v)\right)\right\|^{2} M(m T),
$$

e, como $b(m T, v)=1$, pois $\Gamma$ é não-hiperbólica,

$$
\tilde{\Delta}_{\epsilon}(s, 0)=\int_{0}^{m T} e^{-\int_{0}^{t} \operatorname{divf}\left(\varphi_{r+s}(v)\right) d \tau}<f^{\perp}\left(\varphi_{t+s}(v)\right), g\left(\varphi_{t+s}(v), t+\phi_{0}\right)>d t=M\left(s, \phi_{0}\right),
$$

e o resultado segue do Teorema da Função Implícita.

Observe que, na função $M\left(s, \phi_{0}\right)$ dada no teorema acima, os parâmetros $s$ e $\phi_{0}$ têm a seguinte interpretação geométrica: $s$ está relacionado com o ponto base (ou condição inicial) considerado sobre a órbita periódica ressonante $\Gamma$, pois, para $t=0$ a solução $\varphi_{t+s}(v)$ passa por $\varphi_{s}(v)$; o parâmetro $\phi_{0}$ está relacionado com a seção transversal $\Sigma^{\phi_{0}}$. sobre a qual definimos a transformação de Poincaré $P_{\epsilon}^{m}$.

Assim, fixar $\phi_{0}$ e variar $s$ significa mudar a condição inicial $\varphi_{s}(v)$ considerada sobre $\Gamma$, mantendo-se fixa a seçào transversal $\Sigma \varphi^{\varphi_{0}}$. Por outro lado. fixar $s$ e variar $\phi_{0}$, corresponde a fixar o ponto base sobre $\Gamma$ e considerar a seçào transversal $\Sigma^{\phi_{0}}$ variável. As duas abordagens são equivalentes, como se pode concluir da demonstração do Teorema.

$\mathrm{Na}$ análise a ser realizada na próxima seção considera-se, sem perda de generalidade. $s$ fixo (de fato, considera-se $s=0$ ) e $\phi_{0}$ variável, na função $M\left(s, \phi_{0}\right)$.

A vantagem de se considerar $\phi_{0}$ variando, em vez de $s$, é que tal parâmetro representa a fase da perturbação periódica $g$. Assim, pode-se verificar as hipóteses do teorema analisando-se propriedades da função de perturbação $g$.

O Teorema acima será usado na próxima seção na análise de bifurcaçòes subharmônicas de famílias a dois parâmetros de campos vetoriais polinomiais no plano. 


\subsection{Bifurcações subharmônicas de campos polino- miais com uma reta invariante}

Seja $X$ um campo polinomial de grau $n$, com uma reta invariante, que não contém pontos de equilíbrio, conectando dois pontos de sela hiperbólicos no infinito. Considerandose, sem perda de generalidade, que tal reta é dada pelo eixo- $y$, pode-se escrever a equação diferencial associada a tal campo da seguinte forma

$$
\begin{aligned}
& \dot{x}=x P(x, y) \\
& \dot{y}=Q(x, y),
\end{aligned}
$$

onde $P(x, y)$ e $Q(x, y)$ são polinômios de grau $n-1$ e $n$, respectivamente, com $Q(0, y)>$ $0, \forall y$. É claro, neste caso, que o grau do polinômio $Q(0, y)$ deve ser par, pois caso contrário $Q(0, y)$ teria pelo menos uma raiz real e, consequentemente, pelo menos um equilíbrio sobre o eixo- $y$.

Fazendo uma translação no sistema acima, com objetivo de deixar invariante a reta $\{x=1\}$ em vez do eixo- $y$, obtem-se, após renomear os coeficientes dos polinômios, o sistema

$$
\begin{aligned}
& \dot{x}=(x-1) P(x, y) \\
& \dot{y}=Q(x, y),
\end{aligned}
$$

e, consequentemente, $Q(1, y)>0, \forall y$.

Vimos, no Capítulo 2, que se os pontos de sela são os únicos pontos de equilíbrio no infinito, a compactificação do sistema acima apresenta um ciclo singular no disco de Poincaré, formado por $\gamma(s)$ (que é a compactificação da solução contida na reta invariante $\{x=1\}$ ), $\theta_{1}$ e $\theta_{2}$ (que são os pontos de sela do campo compactificado no bordo do disco de Poincaré) e $\alpha(s)$ (que é o arco de trajetória do campo compactificado contido no bordo do disco) - ver Figura 4.1. 
Suponha que o ciclo singular $\gamma(s) \cup \theta_{1} \cup \alpha(s) \cup \theta_{2}$ é acumulado por órbits periódicas do campo compactificado (Figura 4.1 b)). Tais órbitas correspondem a órbitas periódicas de grande amplitude do campo polinomial no plano.

Seguindo as idéias desenvolvidas no Capítulo 3. considere uma perturbação periódica do campo acima, da forma

$$
\begin{aligned}
& \dot{x}=\tilde{P}(x, y)+\mu+A f(\tilde{\omega} t) \\
& \dot{y}=Q(x, y)+A g(\tilde{\omega} t),
\end{aligned}
$$

onde $\tilde{P}(x, y)=(x-1) P(x, y), f$ e $g$ são funções de classe $C^{k} . k \geq 2$, periódicas de período $T=2 \pi / \tilde{\omega}$, sendo $\mu, A$ parâmetros reais e $\tilde{\omega}=\omega\left(\sqrt{x^{2}+y^{2}}\right)^{n-1}$, $\operatorname{com} \omega>0$ constante. Trata-se de uma perturbação periódica de amplitude $A$ e frequência $\tilde{\omega}$, que depende da posição $(x, y)$, combinada com uma perturbação autônoma, dada pelo parâmetro $\mu$.

Nesta seção analisa-se o efeito da perturbação acima sobre o anel de órbitas periódicas de grande amplitude que se acumulam no ciclo singular infinito. Os métodos apresentados podem ser utilizados também no estudo de outros tipos de perturbaçòes periódicas, como por exemplo,

$$
\begin{aligned}
& \dot{x}=\tilde{P}(x, y)+\mu_{1} f(\dot{w} t) \\
& \dot{y}=Q(x, y)+\mu_{2} g(\dot{w} t) .
\end{aligned}
$$

sendo $\mu_{1}$ e $\mu_{2}$ parâmetros reais. Porém, como o interesse deste trabalho é o estudo de perturbações periódicas como as apresentadas no contexto do Capítulo 3. no que segue será considerado o sistema (4.10)-(4.11).

Como, para o campo polinomial no plano, uma parte de tais órbitas periódicas tende para a reta invariante, e outra porção tende para infinito, de maneira que não se tem controle finito sobre elas, para melhor estudá-las consideremos a compactificação de (4.10)-(4.11) no disco de Poincaré, dada por (conforme Capítulo 2)

$$
\dot{\theta}=\sum_{i=1}^{n} \rho^{i} A_{n-i}(\theta)+\bar{f}(\theta . \rho . \mu . A . \dot{\omega} s)
$$




$$
\dot{\rho}=-\sum_{i=0}^{n} \rho^{i+1} R_{n-i}(\theta)+\bar{g}(\theta, \rho, \mu, A, \tilde{\omega} s),
$$

onde

$$
\begin{gathered}
A_{k}(\theta)=-P_{k}(\cos \theta, \operatorname{sen} \theta) \operatorname{sen} \theta+Q_{k}(\cos \theta, \operatorname{sen} \theta) \cos \theta \\
R_{k}(\theta)=P_{k}(\cos \theta, \operatorname{sen} \theta) \cos \theta+Q_{k}(\cos \theta, \operatorname{sen} \theta) \operatorname{sen} \theta,
\end{gathered}
$$

para $k=0,1, \ldots, n, \operatorname{com} \theta \in[0,2 \pi)$ e $\rho \in[0, \infty)$,

$$
\begin{gathered}
\bar{f}(\theta, \rho, \mu, A, \tilde{\omega} s)=\rho^{n}[A g(\phi) \cos \theta-(\mu+A f(\tilde{\omega} s)) \operatorname{sen} \theta] \\
\bar{g}(\theta, \rho, \mu, A, \tilde{\omega} s)=-\rho^{n+1}[(\mu+A f(\tilde{\omega} s)) \cos \theta+A g(\tilde{\omega} s) \operatorname{sen} \theta],
\end{gathered}
$$

Observe que a derivação acima é relativa a variável independente $s$, que aparece explicitamente nas funções $\bar{f}$ e $\bar{g}$, e representa o tempo do sistema, no disco de Poincaré.

O problema que se coloca aqui é o seguinte: quais das órbitas periódicas contidas no anel que se acumula no ciclo singular infinito, para $\mu=A=0$, persistem como órbitas periódicas do campo perturbado, ao fazermos $\mu \neq 0$ e $A \neq 0$, suficientemente pequenos?

Seguindo as idéias apresentadas na seção anterior, tomando-se $\phi=\tilde{\omega} s$, pode-se considerar o sistema acima como um sistema autônomo numa variedade difeomorfa ao toro sólido $\mathrm{D}^{2} \times \mathrm{S}^{1}$, dado por

$$
\begin{aligned}
& \dot{\theta}=\sum_{i=1}^{n} \rho^{i} A_{n-i}(\theta)+\bar{f}(\theta, \rho, \mu, A, \phi) \\
& \dot{\rho}=-\sum_{i=0}^{n} \rho^{i+1} R_{n-i}(\theta)+\bar{g}(\theta, \rho, \mu, A, \phi) \\
& \dot{\phi}=\omega,
\end{aligned}
$$

onde $\omega>0$, e identificamos $\mathrm{S}^{1}$ com o intervalo $[0,2 \pi)$, considerando tal intervalo como o espaço quociente $\mathrm{IR} / 2 \pi \mathrm{Z}$.

Um esboço do retrato de fase do sistema (4.14)-(4.16), com $\mu=A=0$, no toro sólido, é mostrado na Figura 4.3. As órbitas periódicas que se acumulam no ciclo 
singular infinito se tornam, no espaço de fase estendido $\mathrm{D}^{2} \times \mathrm{S}^{1}$. toros invariantes do sistema.

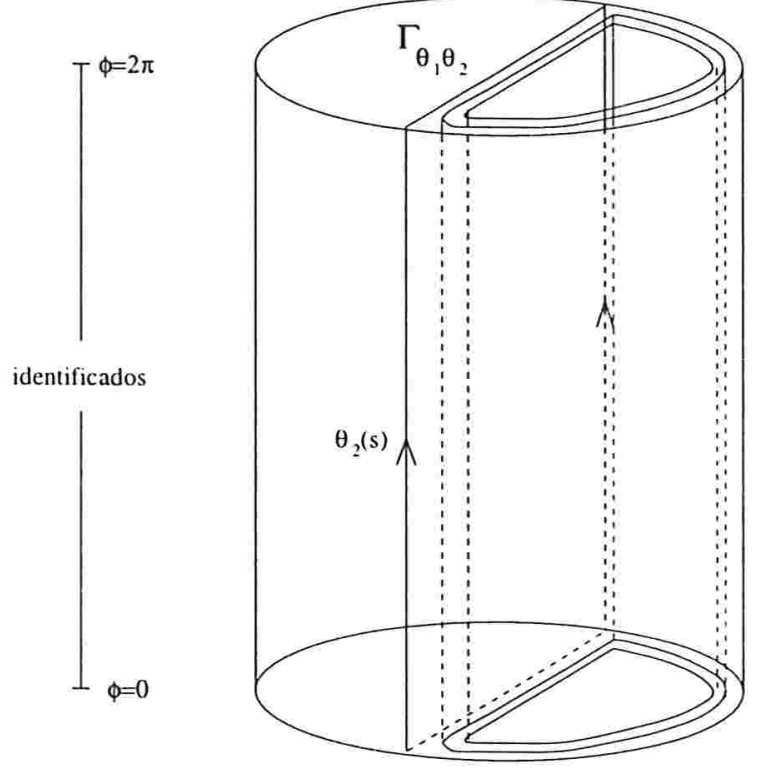

Figura 4.3: Retrato de fase do sistema (4.14)-(4.16), $\operatorname{com} \mu=A=0$. no toro sólido. As órbitas periódicas que se acumulam no ciclo singular infinito se tornam, neste espaço de fase estendido, toros invariantes do sistema.

Estamos agora nas condições apropriadas para aplicar os resultados estabelecidos na seção anterior.

Para tanto, considere o sistema (4.14)-(4.16) escrito na forma

$$
\begin{aligned}
\dot{u} & =F(u)+\mu G_{1}(u)+A G_{2}(u . \phi) \\
\dot{\phi} & =\omega
\end{aligned}
$$

onde $u=(\theta, \rho), \omega>0, \phi \in[0,2 \pi)$, e

$$
\begin{gathered}
F(\theta, \rho)=\left(\sum_{i=1}^{n} \rho^{i} A_{n-i}(\theta),-\sum_{i=0}^{n} \rho^{i+1} R_{n-i}(\theta)\right) . \\
G_{1}(\theta, \rho)=\left(-\rho^{n} \operatorname{sen} \theta \cdot-\rho^{n+1} \cos \theta\right) .
\end{gathered}
$$




$$
G_{2}(\theta, \rho)=\left(\rho^{n}(g(\phi) \cos \theta-f(\phi) \operatorname{sen} \theta),-\rho^{n+1}(f(\phi) \cos \theta+g(\phi) \operatorname{sen} \theta)\right) .
$$

Tomemos a seção transversal ao fluxo do sistema acima, definida por

$$
\Sigma^{\phi_{0}}=\left\{(\theta, \rho, \phi) \mid(\theta, \rho) \in[0,2 \pi) \times[0,+\infty) \quad \epsilon \quad \phi=\phi_{0} \in[0,2 \pi)\right\}
$$

sobre a qual se define a m-ésima iterada da transformação de Poincaré, associada ao campo (4.14)-(4.16),

$$
P_{\mu, A}^{m}: \Sigma^{\phi_{0}} \longrightarrow \Sigma^{\phi_{0}}
$$

dada por

$$
P_{\mu, A}^{m}\left(u_{0}\right)=u\left(m T, u_{0}, \phi_{0}, \mu, A\right),
$$

sendo $u\left(t, u_{0}, \phi_{0}, \mu, A\right)$ solução de

$$
\dot{u}=F(u)+\mu G_{1}(u)+A G_{2}(u, \phi(s)),
$$

com $u\left(0, u_{0}, \phi_{0}, \mu, A\right)=u_{0}$, onde $\phi(s)=\omega s+\phi_{0}$ é a expressão de $\phi$, obtida como solução da equação (4.18), com $\phi(0)=\phi_{0}$. Assim, para $s=0, u_{0} \in \Sigma^{\phi_{0}}$.

É claro assim que os pontos fixos da transformação de Poincaré acima correspondem a subharmônicas de ordem $m$ do sistema (4.14)-(4.16), desde que $P_{\mu, A}^{j}\left(u_{0}\right) \neq u_{0}$ para $1<j<m$.

Em resumo, o sistema $\dot{u}=F(u)$, associado ao sistema compactificado (4.14)-(4.16) $\operatorname{com} \mu=A=0$, possui um anel $\mathcal{A}$ de órbitas periódicas que se acumula no ciclo singular infinito $\theta_{1} \cup \gamma(s) \cup \theta_{2} \cup \alpha(s)$. Estamos interessados em estudar quais destas órbitas periódicas persistem para o sistema perturbado $\dot{u}=F(u)+\mu G_{1}(u)+A G_{2}(u, \phi(s))$. para $\mu$ e $A$ pequenos.

Feitas as considerações acima, damos a seguir três Lemas técnicos que serão usados na prova do Teorema 4.2.4, que é o principal resultado desta seção, estabelecendo regiões no espaço de parâmetros $(\mu, A)$ para as quais o sistema perturbado (4.19) possui órbitas subharmônicas de ordem $m$. O primeiro deles (Lema 4.2.1) estabelece a regularidade do anel $\mathcal{A}$ que se acumula no ciclo singular infinito, para o campo polinomial considerado 
acima. Com o segundo (Lema 4.2.2), mostramos que tal anel contém uma quantidade infinita enumerável de órbitas periódicas ressonantes. O terceiro (4.2.3) é um lema de perturbação.

Lema 4.2.1 O anel $\mathcal{A} \subset \Sigma^{\phi_{0}}$ de órbitas periódicas do sistema (4.19), com $\mu=A=0$, que se acumula no ciclo singular infinito $\gamma(s) \cup \theta_{1} \cup a(s) \cup \theta_{2}$, é regular, em uma vizinhança suficientemente pequena do ciclo.

Prova: Considere a seção transversal ao fluxo do sistema (4.19). com $\mu=A=0$, dada por

$$
\Sigma=\{(\theta, \rho) \mid \theta=\pi \text { e } \rho \in[0, \bar{\rho})\},
$$

com $\bar{\rho}$ suficientemente pequeno (figura 4.4 ).

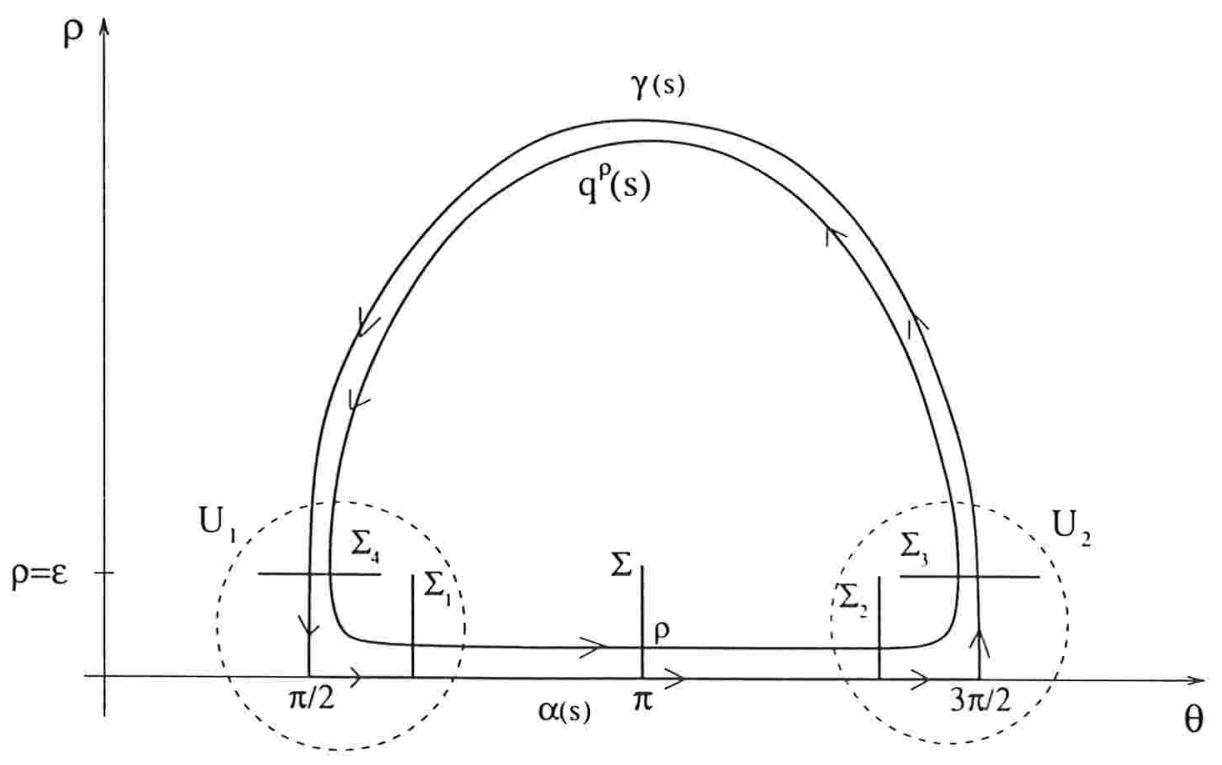

Figura 4.4: Ciclo singular infinito não hiperbólico, visto no plano $(\theta, \rho) \cdot \operatorname{com} \theta \in[0,2 \pi)$ e $\rho \in \mathbb{R}$, juntamente com uma órbita periódica $q^{\rho}(s)$, contida no anel $\mathcal{A}$, seccionada pelas seçòes transversais $\Sigma_{i}$. O eixo- $\theta$ (dado por $\rho=0$ ) corresponde a fronteira do disco de Poincaré.

Seja $q^{\rho}(s)$ a órbita periódica contida no anel $\mathcal{A}$, tal que $q^{\rho}(0)=(\rho, \pi) \in \Sigma$, com 
$\rho \in(0, \bar{\rho})$. Seja $T(\rho)$ o período de $q^{\rho}(s)$. Mostremos que $T^{\prime}(\rho) \neq 0$, para todo $\rho \in(0, \bar{\rho})$ (diminuindo $\bar{\rho}$, se necessário).

Para tanto, considere seções $\Sigma_{i}, \quad i=1,2,3,4$, transversais ao anel $\mathcal{A}$, da seguinte forma (Figura 4.4)

$$
\begin{gathered}
\Sigma_{1}=\left\{(\theta, \rho) \mid \theta=\frac{\pi}{2}+\epsilon \quad \text { e } \quad \rho \in[0, \epsilon)\right\}, \\
\Sigma_{2}=\left\{(\theta, \rho) \mid \theta=\frac{3 \pi}{2}-\epsilon \quad \text { e } \quad \rho \in[0, \epsilon)\right\}, \\
\Sigma_{3}=\left\{(\theta, \rho) \mid \theta \in\left(\frac{3 \pi}{2}-\epsilon, \frac{3 \pi}{2}+\epsilon\right) \quad e \quad \rho=\epsilon\right\}, \\
\Sigma_{4}=\left\{(\theta, \rho) \mid \theta \in\left(\frac{\pi}{2}-\epsilon, \frac{\pi}{2}+\epsilon\right) \quad \epsilon \quad \rho=\epsilon\right\},
\end{gathered}
$$

com $0<\epsilon<\bar{\rho}$.

Como as órbitas periódicas $q^{\rho}(s)$ se acumulam no ciclo $\gamma(s) \cup \theta_{1} \cup \alpha(s) \cup \theta_{2}$, para $\bar{\rho}$ suficientemente pequeno, cada órbita $q^{\rho}(s)$, com $\rho \in(0, \bar{\rho})$, cruza as seções transversais $\Sigma_{i}, i=1,2,3,4$, definidas acima. Assim, pode-se considerar

$$
T(\rho)=T_{1}(\rho)+T_{2}(\rho)+T_{3}(\rho)+T_{4}(\rho),
$$

onde $T_{i}(\rho)$ é o tempo que a órbita $q^{\rho}(s)$ leva para ir de $\Sigma_{i}$ até $\Sigma_{i+1}$ (identificando $\Sigma_{5}$ $\operatorname{com} \Sigma_{1}$, para fechar o ciclo).

Pelo teorema da dependência contínua das soluções com relação a condições iniciais, segue que $T_{1}(\rho)$ e $T_{3}(\rho)$ são funções analíticas de $\rho$, tais que

$$
\lim _{\rho \rightarrow 0^{+}} T_{1}(\rho)=T_{1}(0)<+\infty \quad \text { e } \quad \lim _{\rho \rightarrow 0^{+}} T_{3}(\rho)=T_{3}(0)<+\infty .
$$

Por outro lado, os tempos $T_{2}$ e $T_{4}$ são os tempos de transição de $q^{\rho}(s)$ próximo aos pontos de sela, $\theta_{1}=\left(\theta_{1}, 0\right)$ e $\theta_{2}=\left(\theta_{2}, 0\right)$, do sistema (4.14)-(4.16) no equador, sendo $\theta_{1}=\pi / 2$ e $\theta_{2}=3 \pi / 2$. Como, por hipótese, tais pontos são selas hiperbólicas, e a matriz jacobiana do sistema, calcula nestes pontos é dada por (conforme Capítulo 2, seção 2.2)

$$
\left(\begin{array}{cc}
A_{n}^{\prime}\left(\theta_{i}\right) & A_{n-1}\left(\theta_{i}\right) \\
0 & -R_{n}\left(\theta_{i}\right)
\end{array}\right),
$$


com $i=1,2$, por um Teorema de Hartman ([17], pg. 258), existem vizinhanças $U_{1}$ e $U_{2}$ de $\theta_{1}$ e $\theta_{2}$, nas quais o sistema é diferenciavelmente conjugado a

$$
\begin{aligned}
& \dot{\theta}=A_{n}^{\prime}\left(\theta_{1}\right)(\theta-\pi / 2) \\
& \dot{\rho}=-R_{n}\left(\theta_{1}\right) \rho,
\end{aligned}
$$

e

$$
\begin{aligned}
& \dot{\theta}=f_{n}^{\prime}\left(\theta_{2}\right)(\theta-3 \pi / 2) \\
& \dot{\rho}=-R_{n}\left(\theta_{2}\right) \rho,
\end{aligned}
$$

onde $-R_{n}\left(\theta_{1}\right)<0<A_{n}^{\prime}\left(\theta_{1}\right)$ e $A_{n}^{\prime}\left(\theta_{2}\right)<0<-R_{n}\left(\theta_{2}\right)$ sào os autovalores do sistema nos pontos de equilíbrio $\theta_{1}$ e $\theta_{2}$. respectivamente.

Escolhendo $\epsilon$ fixo, suficientemente pequeno, de forma que $\Sigma_{1}, \Sigma_{4} \subset U_{1}$ e $\Sigma_{2}, \Sigma_{3} \subset U_{2}$ (Figura 4.4), tem-se entào, das segundas equaçòes dos sistemas acima, que o tempo de transição próximo aos pontos de sela. em função de $\rho$, é dado por

$$
T_{2}(\rho)=-\frac{1}{R_{n}\left(\theta_{2}\right)} \ln \left(\frac{\epsilon}{\rho_{2}(\rho)}\right)>0 \quad \epsilon \quad T_{4}(\rho)=-\frac{1}{R_{n}\left(\theta_{1}\right)} \ln \left(\frac{\rho_{1}(\rho)}{\epsilon}\right)>0,
$$

onde $\rho_{1}(\rho)$ é o ponto onde a órbita $q^{\rho}(s)$ cruza a seção transversal $\Sigma_{1}, \rho_{2}(\rho)$ é o ponto onde ela cruza $\Sigma_{2}$, sendo $\rho_{1}$ e $\rho_{2}$ funçòes diferenciáveis de $\rho$. com

$$
\lim _{\rho \rightarrow 0^{+}} \rho_{1}(\rho)=\lim _{\rho \rightarrow 0^{+}} \rho_{2}(\rho)=0
$$

É claro assim, da expressão (4.22) que

$$
\lim _{\rho \rightarrow 0^{+}} T_{2}(\rho)=+\infty \quad \epsilon \quad \lim _{\rho \rightarrow 0^{+}} T_{4}(\rho)=+\infty .
$$

Além disso, derivando-se $T_{2}$ e $T_{4}$ com relação a $\rho$ tem-se

$$
T_{2}^{\prime}(\rho)=\frac{\rho_{2}^{\prime}(\rho)}{R_{n}\left(\theta_{2}\right) \rho_{2}(\rho)}<0 \quad \text { e } \quad T_{4}^{\prime}(\rho)=-\frac{\rho_{1}^{\prime}(\rho)}{R_{n}\left(\theta_{1}\right) \epsilon \rho_{1}(\rho)}<0,
$$

donde segue que

$$
\lim _{\rho \rightarrow 0^{+}} T_{2}^{\prime}(\rho)=-\infty \quad \epsilon \quad \lim _{\rho \rightarrow 0^{+}} T_{4}^{\prime}(\rho)=-\infty
$$


De (4.20), (4.23) e (4.24) segue que, para $\rho$ suficientemente pequeno, $T^{\prime}(\rho)<0$ e, além disso, $\lim _{\rho \rightarrow 0^{+}} T(\rho)=+\infty$, o que finaliza a prova.

Do Lema acima segue que o gráfico da função período, $T(\rho)$, tem o aspecto mostrado na figura 4.5. Usando então este Lema, provamos o seguinte resultado.

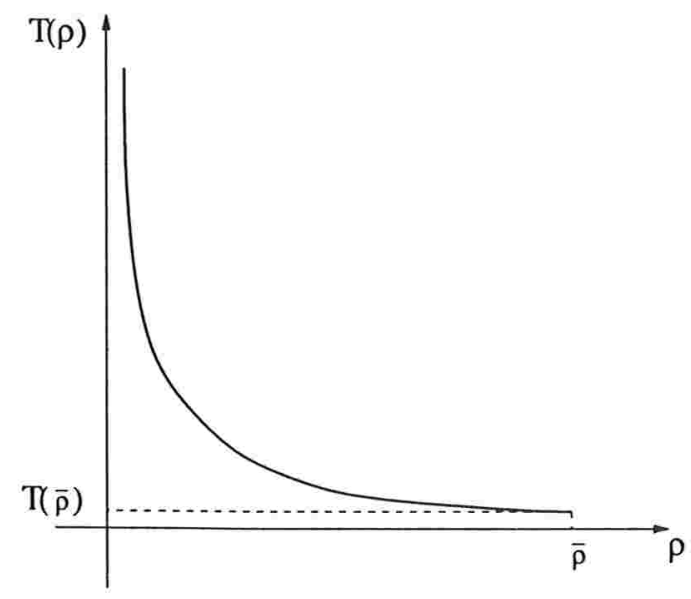

Figura 4.5: Esboço do gráfico da função período, $T(\rho)$, definida em uma seção transversal ao anel de órbitas periódicas que se acumula no ciclo singular infinito.

Lema 4.2.2 Para $\bar{\rho}$ suficientemente pequeno, existe uma sequência $\left\{\rho_{m_{1}}\right\}_{i \in \mathbb{N}} \subset(0, \bar{\rho})$, com $\lim _{i \rightarrow+\infty} \rho_{m_{i}}=0$, a qual corresponde uma família $\left\{q^{\rho_{m_{i}}}(s)\right\}_{i \in \mathbb{N}}$, de órbitas periódicas do sistema (4.19), com $\mu=A=0$, contidas no ant $\mathcal{A}$, tais que o período $T\left(\rho^{m_{i}}\right)$ de $q^{\rho_{m_{i}}}(s)$ satisfaz, para cada $i$, a seguinte relaçào de ressonância com o periodo $2 \pi / \omega$ da função de perturbação $G_{2}(u, \phi(s))$

$$
T\left(\rho_{m_{i}}\right)=m_{i} \frac{2 \pi}{\omega},
$$

onde os $m_{i}$ são números naturais tais que $\lim _{i \rightarrow+\infty} m_{i}=+\infty$.

Prova: Segue imediatamente do lema anterior, já que $T(\rho)$ é uma função contínua, que tende para infinito, assumindo todos os valores do intervalo $[T(\bar{\rho}),+\infty)$. Além disso, 
como $\lim _{i \rightarrow+\infty} \rho_{m_{1}}=0$, e $T\left(\rho_{m_{1}}\right) \rightarrow+\infty$ se $\rho_{m_{1}} \rightarrow 0$, da relação de ressonância (4.25) segue que $m_{i} \rightarrow+\infty$, quando $i \rightarrow+\infty$.

Lema 4.2.3 Seja $q^{\rho}(s)$, com $\rho \in(0, \bar{\rho}), \bar{\rho}$ suficientemente pequeno, uma órbita periódica de período $T(\rho)$ do sistema (4.19), com $\mu=A=0$. contida no anel $\mathcal{A}$. Entào. para $\mu, A \neq 0$ suficientemente pequenos. existe uma órbita $q_{\mu, A}^{\rho}(s)$, não necessariamente periódica, que pode ser expressa da forma

$$
q_{\mu, A}^{\rho}(s)=q^{\rho}(s)+\mu q_{1}^{\rho}(s)+A q_{2}^{\rho}(s)+\text { h.o.t }
$$

uniformemente em $s \in[0, T(\rho)]$, para todo $\rho \in(0, \bar{\rho})$. onde h.o.t. indica termos de grau maior ou igual a dois em $\mu$ e A..

Prova: A prova está feita com detalhes em [15], pg.193, e usa a estrutura geométrica das soluções do sistema na vizinhança do ciclo singular infinito $\gamma(s) \cup \theta_{1} \cup \alpha(s) \cup \theta_{2}$. especialmente a persistência das variedades invariantes locais dos pontos de sela no infinito, $\theta_{1}$ e $\theta_{2}$.

Com os Lemas acima e usando os resultados da seção anterior, provamos o seguinte Teorema,

Teorema 4.2.4 Considere o sistema (4.19), dado por

$$
\dot{u}=F(u)+\mu G_{1}(u)+A G_{2}\left(u \cdot \omega s+\phi_{0}\right) .
$$

Suponha que, para $\mu=A=0$, tal sistema possui um ciclo singular $\gamma(s) \cup \theta_{1} \cup a(s) \cup \theta_{2}$. acumulado por um anel $\mathcal{A}$ de órbitas periódicas. Sejam as funçôes

$$
\begin{gathered}
S_{m}=\int_{0}^{m T} e^{-\int_{0}^{s} \operatorname{div} F\left(q^{\rho_{m}}(\tau)\right) d \tau}<F^{\perp}\left(q^{\rho_{m}}(s)\right), G_{1}\left(q^{\rho_{m}}(s)\right)>d s \\
M_{m}\left(\phi_{0}\right)=\int_{0}^{m T} e^{-\int_{0}^{s} \operatorname{div} F\left(q^{\rho_{m}}(\tau)\right) d \tau}<F^{\perp}\left(q^{\rho_{m}}(s)\right) \cdot G_{2}\left(q^{\rho_{m}}(s), \omega \cdot s+\phi_{0}\right)>d s .
\end{gathered}
$$


onde $q^{\rho_{m}}(s)$ é uma órbita periódica ressonante, contida no anel $\mathcal{A}$ e $F, G_{1}$ e $G_{2}$ são as funções do sistema (4.19), sendo $F^{\perp}$ o ortogonal de $F$.

Se $S_{m}$ for diferente de zero e $M_{m}$ possuir somente dois pontos críticos, um de máximo e um mínimo, dados por $\phi_{\max }$ e $\phi_{\min }$, tais que $M^{\prime \prime}\left(\phi_{\max }\right) \neq 0$ e $M^{\prime \prime}\left(\phi_{\min }\right) \neq 0$, e, além disso, $M\left(\phi_{\min }\right)<0<M\left(\phi_{\max }\right)$, então valem os seguintes resultados: existem curvas diferenciáveis $A_{1}^{m}(\mu)$ e $A_{2}^{m}(\mu)$, para $\mu$ suficientemente pequeno, que dividem o espaço de parâmetros $(\mu, A)$ em regiões disjuntas $R_{1}$ e $R_{2}$ (Figura 4.6) tais que:

i) para $(\mu, A)$ em $R_{1}$, o sistema perturbado possui 2 órbitas subharmônicas de ordem $m$, que bifurcam da órbita ressonante $q^{\rho_{m}}(s)$;

ii) para $(\mu, A)$ em $R_{2}$, não existem órbitas subharmônicas que bifurcam de $q^{\rho_{m}}(s)$;

iii) para $(\mu, A)$ no bordo das regiões $R_{1}$ e $R_{2}$, ou seja, sobre as curvas $A_{1}^{m}(\mu)$ e $A_{2}^{m}(\mu)$, existe uma única órbita subharmônica de ordem $m$ que bifurca de $q^{\rho_{m}}(s)$.

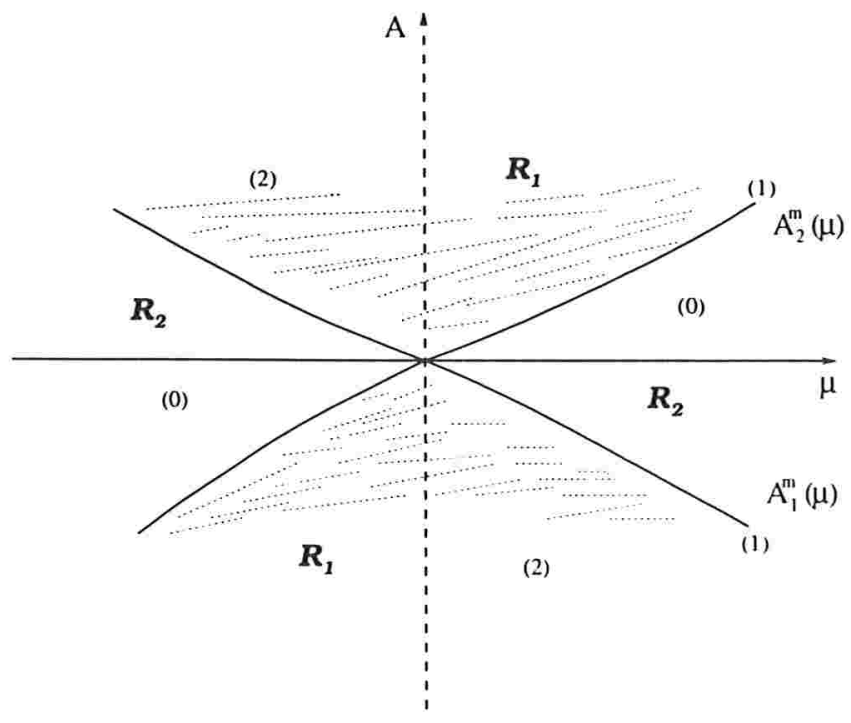

Figura 4.6: Regiões $R_{1}$ e $R_{2}$ no espaço de parámetros $(\mu, A)$, definidas no enunciado do Teorema 4.2.4. O número entre parêntesis indica a quantidade mínima de subharmonicas de ordem $m$ do sistema, para $(\mu, A)$ pertencente à região indicada. 
Observação 4.2.5 As funçōes $S_{m} \in M_{m}\left(\phi_{0}\right)$ que aparecem no enunciado do teorema acima correspondem à função $M\left(s . \phi_{0}\right)$. com $s=0$. do enunciado do Teorema 4.1.6. $S_{m}$ independe de $\phi_{0}$ pois está relacionada à parte autônoma da perturbação, enquanto que $M_{m}\left(\phi_{0}\right)$ está relacionada à parte periódica (ver prova do Teorema).

Prova do Teorema: Considere a transformação de Poincaré $P_{\mu, A}^{m}(u): \Sigma^{\phi_{0}} \rightarrow \Sigma^{\phi_{0}}$, associada ao sistema (4.19). dada por

$$
P_{\mu, A}^{m}(u)=u\left(m T, u, \phi_{0}, \mu, A\right),
$$

onde $u$ é a soluçào de $\dot{u}=F(u)+\mu G_{1}(u)+A G_{2}\left(u, \omega s+\phi_{0}\right), \operatorname{com} u\left(0, u, \phi_{0}, \mu, A\right)=$ $u \in \Sigma^{\phi_{0}}$, sendo $\Sigma^{\phi_{0}}=\left\{(u, \phi) \mid u \in \mathbb{R}^{2}\right.$ e $\left.\phi=\phi_{0} \in[0,2 \pi)\right\}$.

Expandindo a função acima em Série de Taylor, em relação aos parâmetros $\mu$ e $A$, em torno de $\mu=A=0$ tem-se

$$
P_{\mu, A}^{m}(u)=u\left(m T, u, \phi_{0}, 0,0\right)+u_{\mu}\left(m T . u, \phi_{0}, 0,0\right) \mu+u_{A}\left(m T, u, \phi_{0}, 0,0\right) . A+R\left(\phi_{0} \cdot \mu, A\right) .
$$

onde $R\left(\phi_{0}, \mu, A\right)=a\left(\dot{\phi}_{0}, \mu, A\right) \mu^{2}+b\left(\dot{\phi}_{0}, \mu, A\right) \mu A+c\left(\phi_{0}, \mu, A\right) \cdot A^{2}$, sendo $a, b, c$ funções diferenciáveis, com $a\left(\phi_{0}, 0,0\right)=b\left(\phi_{0} .0,0\right)=c\left(\phi_{0}, 0.0\right)=0$

Supondo que $u$ pertence a uma das órbitas periódicas ressonantes, digamos $q^{\rho_{m}}(s)$. dadas pelo Lema 4.2 .2 , tem-se $u\left(m T . u, \varphi_{0}, 0,0\right)=u$. donde segue que

$$
P_{\mu, A}^{m}(u)-u=u_{\mu}\left(m T, u, \phi_{0}, 0.0\right) \mu+u_{A}\left(m T . u, \phi_{0}, 0.0\right) \mathcal{A}+R\left(\phi_{0}, \mu, \mathcal{A}\right)
$$

Chamando

$$
\Delta\left(\phi_{0}, \mu, A\right):=u_{\mu}\left(m T, u, \phi_{0}, 0,0\right) \mu+u_{A}\left(m T, u, \phi_{0}, 0,0\right) A+R\left(\phi_{0}, \mu . A\right),
$$

tem-se que os pontos fixos de $P_{\mu, A}^{m}$ são dados pelos zeros da funçào $\Delta$.

Pelo Teorema 4.1.6, da seçào anterior. juntamente com o Lema 4.2.1. que garante que o anel de órbitas periódicas $\mathcal{A}$ do sistema (4.19) é regular. tem-se que, para $\mu$ e 
$A$ suficientemente pequenos, os zeros da função $\Delta$ definida acima dependem apenas de sua projeção na direção $f^{\perp}\left(q^{\rho_{m}}(s)\right)$, ou seja, dependem apenas das componentes de $u_{\mu}$ e $u_{A}$ na direção de $f^{\perp}\left(q^{\rho_{m}}(s)\right)$, onde $u_{\mu}$ e $u_{A}$ são as soluções das equações variacionais associadas a (4.19), obtidas derivando-se o sistema com relação a $\mu$ e $A$, respectivamente.

Assim, usando-se o Lema 4.1.4, tem-se

$$
<\Delta\left(\phi_{0}, \mu, A\right), f^{\perp}\left(q^{\rho_{m}}(s)\right)>:=S_{m} \mu+M_{m}\left(\phi_{0}\right) A+R\left(\phi_{0}, \mu, A\right)
$$

onde,

$$
\begin{gathered}
S_{m}=\int_{0}^{m T} e^{-\int_{0}^{s} \operatorname{div} F\left(q^{\rho_{m}}(\tau)\right) d \tau}<F^{\perp}\left(q^{\rho_{m}}(s)\right), G_{1}\left(q^{\rho_{m}}(s)\right)>d s, \\
M_{m}\left(\phi_{0}\right)=\int_{0}^{m T} e^{-\int_{0}^{s} \operatorname{div} F\left(q^{\rho_{m}}(\tau)\right) d \tau}<F^{\perp}\left(q^{\rho_{m}}(s)\right), G_{2}\left(q^{\rho_{m}}(s), \omega s+\phi_{0}\right)>d s,
\end{gathered}
$$

que são as projeções de $u_{\mu}$ e $u_{A}$, respectivamente, na direção $f^{\perp}\left(q^{\rho_{m}}(s)\right)$.

Colocando $\mu$ em evidência na expressão acima obtem-se

$$
<\Delta\left(\phi_{0}, \mu, A\right), f^{\perp}\left(q^{\rho_{m}}(s)\right)>:=\mu\left[S_{m} \mu+M_{m}\left(\phi_{0}\right) \eta+\tilde{R}\left(\phi_{0}, \mu, \eta\right)\right]:=\mu \tilde{\Delta}\left(\phi_{0}, \mu, \eta\right)
$$

onde $\eta=A / \mu$,

$$
\tilde{R}\left(\phi_{0}, \mu, \eta\right)=a\left(\phi_{0}, \mu, \eta \mu\right) \mu+b\left(\phi_{0}, \mu, \eta \mu\right) \mu \eta+c\left(\phi_{0}, \mu, \eta \mu\right) \mu \eta^{2}=O(\mu)
$$

e, desse modo, para $\mu \neq 0$ suficientemente pequeno, os zeros da projeção da função $\Delta\left(\phi_{0}, \mu, A\right)$ na direção $f^{\perp}\left(q^{\rho_{m}}(s)\right)$ coincidem com os zeros da função

$$
\tilde{\Delta}\left(\phi_{0}, \mu, \eta\right)=S_{m} \mu+M_{m}\left(\phi_{0}\right) \eta+O(\mu)
$$

Por hipótese, $S_{m} \neq 0$ e $M_{m}\left(\phi_{0}\right)$ possui pontos de máximo e mínimo não degenerados, com $M_{m}\left(\phi_{\min }\right)<0<M_{m}\left(\phi_{\max }\right)$. Supondo, sem perda de generalidade, que $S_{m}>0$, e usando a expressão da função $\tilde{\Delta}$, vamos determinar as regiões $R_{1}$ e $R_{2}$ do enunciado do Teorema. $\tilde{\Delta}\left(\phi_{0}, \mu, \eta\right)=0$ se, e somente se,

$$
S_{m}+M_{m}\left(\phi_{0}\right) \eta+O(\mu)=0
$$


Por outro lado,

$$
M_{m}\left(\phi_{\min }\right) \leq M_{m}\left(\phi_{0}\right) \leq M_{m}\left(\phi_{\max }\right)
$$

Das relações acima, isolando $M\left(\phi_{0}\right)$ em $(4.26)$ e sendo $\eta=A / \mu$, segue que

$$
M_{m}\left(\phi_{\min }\right) \leq-\frac{S_{m}}{\eta}-O\left(\mu^{2}\right) \leq M_{m}\left(\phi_{\max }\right)
$$

donde tem-se as relações

$$
\eta \geq-\frac{S_{m}}{M_{m}\left(\phi_{\min }\right)}-O(\mu) \quad \text { ou } \quad \eta \geq-\frac{S_{m}^{\prime}}{M_{m}\left(\phi_{\max }\right)}-O(\mu) \quad \text { se } \eta>0,
$$

e

$$
\eta \leq-\frac{S_{m}}{M_{m}\left(\phi_{\min }\right)}-O(\mu) \quad \text { ou } \quad \eta \leq-\frac{S_{m}}{M_{m}\left(\phi_{\max }\right)}-O(\mu) \quad \text { se } \eta<0,
$$

Dentre as quatro relações acima, $\eta \geq-\frac{S_{m}}{M_{m}\left(\phi_{\max }\right)}-O(\mu)$ e $\eta \leq-\frac{S_{m}}{M_{m}\left(\phi_{\min }\right)}-O(\mu)$ são sempre satisfeitas, devido às hipóteses. Usando as outras duas relações e voltando a mudança de variáveis $\eta=A / \mu$, obtem-se, para $\mu>0$,

$$
A \geq-\frac{S_{m}}{M_{m}\left(\phi_{\min }\right)} \mu-O\left(\mu^{2}\right)
$$

$\mathrm{e}$

$$
A \leq-\frac{S_{m}}{M_{m}\left(\phi_{\max }\right)} \mu-O\left(\mu^{2}\right)
$$

Por outro lado, se $\mu<0$ obtem-se

$$
A \leq-\frac{S_{m}}{M_{m}\left(\phi_{\min }\right)} \mu-O\left(\mu^{2}\right)
$$

$\mathrm{e}$

$$
A \geq-\frac{S_{m}}{M_{m}\left(\phi_{\max }\right)} \mu-O\left(\mu^{2}\right)
$$

As quatro relações acima, para o parâmetro $A$ como função de $\mu$, determinam as regiões $R_{1}$ e $R_{2}$ (Figura 4.6), no espaço de parâmetros, para as quais existe pelo menos uma órbita subharmônica de ordem $m$ para o sistema (4.19), que bifurca da órbita 
periódica ressonante $q^{\rho_{m}}(s)$. Tais regiões têm como fronteira as curvas $A_{1}^{m}(\mu)$ e $A_{2}^{m}(\mu)$, com $\mu$ suficientemente pequeno, dadas por

$$
\begin{aligned}
& A_{1}^{m}(\mu)=-\frac{S_{m}}{M_{m}\left(\phi_{\max }\right)} \mu-O\left(\mu^{2}\right), \\
& A_{2}^{m}(\mu)=-\frac{S_{m}}{M_{m}\left(\phi_{\min }\right)} \mu-O\left(\mu^{2}\right),
\end{aligned}
$$

que independem da seção transversal $\Sigma^{\phi_{0}}$, onde está definida a transformação de Poincaré $P_{\mu, A}^{m}$. Dependem apenas dos valores de $S_{m}, M_{m}\left(\phi_{\min }\right)$ e $M_{m}\left(\phi_{\max }\right)$.

Mostremos agora, usando as hipóteses $M^{\prime \prime}\left(\phi_{\max }\right)<0$ e $M^{\prime \prime}\left(\phi_{\min }\right)>0$, que se $(\mu, A)$ pertence ao interior das regiões $R_{1}$ e $R_{2}$, então existem duas órbitas subharmônicas de ordem $m$ que bifurcam da órbita periódica ressonante $q^{\rho_{m}}(s)$ e, se $(\mu, A)$ está sobre uma das curvas $A_{1}^{m}(\mu)$ ou $A_{2}^{m}(\mu)$, existe somente uma subharmônica de ordem $m$.

Expandido $M\left(\phi_{0}\right)$ em Série de Taylor em $\phi_{0}$, em torno de $\phi_{0}=\phi_{\max }$ e substituindo na expressão de $\tilde{\Delta}\left(\phi_{0}, \mu, \eta\right)$, obtem-se

$$
\tilde{\Delta}\left(\phi_{0}, \mu, \eta\right)=S_{m}+M_{m}\left(\phi_{\max }\right) \eta+\beta \eta\left(\phi_{0}-\phi_{\max }\right)^{2}+O\left(\left(\phi_{0}-\phi_{\max }\right)^{3}\right) \eta+O(\mu),
$$

$\operatorname{com} \beta=M^{\prime \prime}\left(\phi_{\max }\right) / 2<0$. Assim, se $(\mu, A) \in A_{1}^{m}(\mu)$, então $\eta=-S_{m} / M_{m}\left(\phi_{\max }\right)-$ $O(\mu)$, que, substituído na expressão acima, implica em que $\tilde{\Delta}\left(\phi_{0}, \mu, \eta\right)=0$ se, e somente se,

$$
\beta \eta\left(\phi_{0}-\phi_{\max }\right)^{2}+O\left(\left(\phi_{0}-o_{\max }\right)^{3}\right) \eta=0 .
$$

Como, para $\mu$ suficientemente pequeno, $\beta \eta \neq 0$, segue que a expressão acima se anula se, e somente se, $\phi_{0}-\phi_{\max }=0$. Portanto, se $(\mu . A) \in A_{1}^{m}(\mu), \phi_{0}=\phi_{\max }$ é o único zero de $\tilde{\Delta}\left(\phi_{0}\right)$, que corresponde a uma única órbita periódica subharmônica de ordem $m$, bifurcando de $q^{\rho_{m}}(s)$.

Procedendo-se de maneira análoga, expandindo $M\left(\phi_{0}\right)$ em torno de $\phi_{\min }$, mostra-se que, se $(\mu, A)$ está sobre $A_{2}^{m}(\mu)$, então $\phi_{0}=\phi_{\min }$ é o único zero de $\tilde{\Delta}\left(\phi_{0}\right)$.

Suponha agora que $(\mu, A)$ pertence ao interior da região $R_{2}$. A igualdade

$$
\tilde{\Delta}\left(\phi_{0}, \mu, \eta\right)=S_{m}+M_{m}\left(\phi_{\max }\right) \eta+\beta \eta\left(\phi_{0}-\phi_{\max }\right)^{2}+O\left(\left(\phi_{0}-\phi_{\max }\right)^{3}\right) \eta+O(\mu)=0,
$$


equivale a

$$
\beta \eta \phi_{0}{ }^{2}-2 \beta \eta \phi_{0} \phi_{\max }+\beta \eta \phi_{\max }{ }^{2}+S_{m}+M_{m}\left(\phi_{\max }\right) \eta+O\left(\left(\phi_{0}-\phi_{\max }\right)^{3}\right) \eta+O(\mu)=0 .
$$

A equação acima, como função de $\phi_{0}$, tem duas raízes reais se, e somente se, seu discriminante for estritamente positivo. o que, após alguns cálculos algébricos simples, equivale a

$$
-4 \beta \eta\left[S_{m}+M_{m}\left(\phi_{\max }\right) \eta+O\left(\left(\phi_{0}-\phi_{\max }\right)^{3}\right) \eta+O(\mu)\right]>0
$$

Como, para $\mu$ suficientemente pequeno, $\beta \eta>0$, a desigualdade acima é verdadeira se, e somente se,

$$
\eta<-S_{m} / M_{m}\left(\phi_{\max }\right)-O\left(\left(\phi_{0}-\phi_{\max }\right)^{3}\right) \eta-O(\mu)
$$

Tomando $\left(\phi_{0}-\phi_{\max }\right)$ da ordem de $\mu^{2 / 3}$, como $\eta=A / \mu$, segue que $O\left(\left(\phi_{0}-\phi_{\max }\right)^{3}\right) \eta=$ $O(\mu)$. Assim, a desigualdade acima fica da forma

$$
\eta<-S_{m} / M_{m}\left(\phi_{\max }\right)-O(\mu)
$$

que, para $\mu$ suficientemente pequeno. equivale à hipótese de $(\mu, A)$ pertencer ao interior da região $R_{2}, \operatorname{com} A>0$.

De modo análogo, expandindo $\dot{j}$ em torno de $\phi_{0}=\phi_{\min }$. mostra-se que a equação $\tilde{\Delta}\left(\phi_{0}, \mu, \eta\right)=0$ possui duas raízes reais se $(\mu, A)$ pertence ao interior da regiào $R_{2}$, com $A<0$.

As duas raízes de $\tilde{\Delta}=0$ correspondem a $2 m$ pontos fixos da transformação de Poincaré $P_{\mu, A}^{m}$, correspondentes às duas órbitas subharmônicas de ordem $m$ do sistema (4.19), que bifurcam da órbita ressonante $q^{\rho_{m}}(s)$. Fica assim provado o Teorema.

Observação 4.2.6 As hipóteses sobre a funçào .M(o $\left.o_{0}\right)$ que aparecem no teorema acima estão diretamente relacionadas com propriedades da funçào periódica de perturbação $G_{2}\left(u, \omega s+\phi_{0}\right)$ do sitema (4.19). como se pode l'er de sua expressão. 
Com o auxílio da Figura 4.6, vejamos o cenário descrito pelo Teorema acima. Fixando-se um valor de $\mu$, digamos positivo e suficientemente pequeno, e variandose $A$ na direção vertical, desde $A=0$, tem-se: inicialmente, para $A$ próximo de zero, o sistema não apresenta órbitas periódicas subharmônicas bifurcando de $q^{\rho_{m}}(s)$; quando $A$ atinge o valor $A=A_{1}^{m}(\mu)$, passa a existir uma única subharmônica de ordem $m$; finalmente, para $A>A_{1}^{m}(\mu)$ existem 2 subharmônicas de ordem $m$ que bifurcam de $q^{\rho_{m}}(s)$. Estas órbitas correspondem a pontos fixos da Transformação de Poincaré $P_{\mu, A}^{m}$, com $\mu$ fixo, suficientemente pequeno. Tal cenário é comumente encontrado no estudo de famílias a um parâmetro de difeomorfismos, dado neste caso por $P_{\mu, A}^{m}$. Quando o parâmetro $A$ atravessa a curva $A_{1}^{m}(\mu)$, ocorre uma bifurcação do tipo sela-nó para o difeomorfismo, originando as duas órbitas subharmônicas.

Para ilustrar a aplicabilidade do Teorema acima, na próxima seção será feita a análise de uma perturbação periódica do campo vetorial quadrático, contendo uma reta invariante e dois ciclos singulares infinitos, considerado no Capítulo 3, no caso em que tais ciclos são acumulados por órbitas periódicas.

\subsection{Estudo do caso quadrático}

Considere a família de campos polinomiais quadráticos

$$
\begin{aligned}
\dot{x} & =x y-y \\
\dot{y} & =\alpha+m x+n y+a x^{2}+b x y+c y^{2} .
\end{aligned}
$$

e suponha que o sistema satisfaz as hipóteses (H1)-(H3) da Proposição 3.1.1 do Capítulo 3. Relembrando, tal sistema possui somente dois pontos de equilíbrio no infinito, que são pontos de sela hiperbólicos, $\theta_{1}$ e $\theta_{2}$, conectados por uma solução heteroclínica $\gamma(s)$, que é uma reparametrização da solução contida na reta invariante $\{x=1\}$, formando os ciclos singulares infinitos $\gamma(s) \cup \theta_{1} \cup \pm \alpha(s) \cup \theta_{2}$ (Figura 4.7). Além disso, o campo 
possui dois pontos de equilíbrio no eixo- $x$, que são focos ou centros.

Lema 4.3.1 O sistema (4.27)-(4.28) possui dois centros no eiro-x se. $\epsilon$ somente se. $n=b=0$.

Prova: O sistema acima possui dois pontos de equilíbrio no eixo- $x$, dados por

$$
E_{1,2}=\frac{-m \pm \sqrt{m^{2}-4 a \alpha}}{2 a}
$$

sendo que o traço da matriz jacobiana do sistema, calculada nestes pontos, é igual a zero se, e somente se, $n=b=0$, o que é uma condição necessária para que $E_{1}$ e $E_{2}$ sejam centros.

Fazendo a mudança de variáveis $(x, y) \rightarrow(x,-y)$ segue que. se $b=n=0$, o campo é simétrico em relaçào ao eixo- $x$ e, portanto, $E_{1}$ e $E_{2}$ são centros.

O retrato de fase da compactificação de (4.27)-(4.28), $\operatorname{com} n=b=0$. no disco de Poincaré, tem o aspecto mostrado na Figura 4.7.

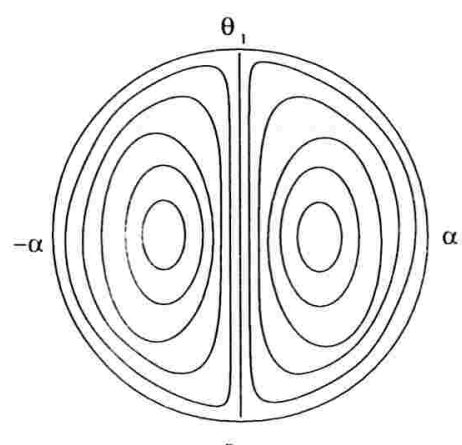

$\theta_{2}$

Figura 4.7: Retrato de fase da compactificação do sistema (4.2 $)$-(4.28), no disco de Poincaré.

Considere agora a seguinte perturbaçào periódica do sistema

$$
\begin{aligned}
& \dot{x}=x y-y+\mu+A\left(a_{1} \cos \dot{\omega} t+a_{2} \operatorname{sen} \dot{\omega} t\right) \\
& \dot{y}=a+m x+a x^{2}+c y^{2}+A\left(b_{1} \cos \dot{\omega} t+b_{2} \operatorname{sen} \dot{\omega} t\right) .
\end{aligned}
$$


onde $\mu$ e $A$ são parâmetros reais, $\tilde{\omega}=\omega \sqrt{x^{2}+y^{2}}$, $\operatorname{com} \omega>0$ constante.

Façamos uma análise das bifurcações subharmônicas que ocorrem com as órbitas periódicas do sistema, em decorrência desta perturbação. Será considerado somente o anel de órbitas periódicas contido na porção direita do disco de Poincaré (isto é, $-\pi / 2 \leq \theta \leq \pi / 2$ - Figura 4.7), já que o estudo do outro anel se faz de maneira análoga. Aplicaremos o Teorema 4.2.4 da seção anterior.

Para aplicar os resultados da seção anterior, em primeiro lugar é necessário observar que, apesar de o anel de órbitas periódicas ter como limite, por um lado o ponto de equilíbrio, e pelo outro o ciclo singular infinito $\gamma(s) \cup \theta_{1} \cup+\alpha(s) \cup \theta_{2}$, estendendo-se por toda a porção direita do disco, não se pode garantir que ele é regular. Ou seja, não se pode garantir que a função período seja estritamente monótona, em todo o anel. De fato, trata-se de um problema em aberto saber o número de pontos críticos da função período, definida em um anel de órbitas periódicas de um campo polinomial no plano, no caso em que tal anel é ilimitado, ou seja, as órbitas periódicas tendem, como conjunto, para infinito (conforme [6]).

Assim, como feito anteriormente, analisaremos as bifurcações subharmônicas somente com relação às órbitas periódicas de grande amplitude, isto é, para a parte do anel que se acumula no ciclo singular infinito. Para tais órbitas, vale o Lema 4.2.1 e também o Lema 4.2.2, o que garante que elas formam um anel regular, no qual existe uma quantidade infinita enumerável de órbitas periódicas ressonantes. Nos cálculos que seguem consideraremos $q^{\rho_{m}}(s)$ uma destas órbitas ressonantes.

A compactificação do sistema (4.29)-(4.30), em coordenadas $(\theta, \rho)$ é dada por

$$
\begin{array}{r}
\dot{\theta}=a \cos ^{3} \theta+(c-1) \operatorname{sen}^{2} \theta \cos \theta+\rho\left(m \cos ^{2} \theta+\operatorname{sen}^{2} \theta\right)+\rho^{2} \alpha \cos \theta \\
-\mu \rho^{2} \operatorname{sen} \theta+A \rho^{2}\left[\left(b_{1} \cos \omega s+b_{2} \operatorname{sen} \omega s\right) \cos \theta-\left(a_{1} \cos \omega s+a_{2} \operatorname{sen} \omega s\right) \operatorname{sen} \theta\right] \\
\dot{\rho}=-\rho\left[\operatorname{csen}^{3} \theta+(a+1) \cos ^{2} \theta \operatorname{sen} \theta+\rho(m-1) \operatorname{sen} \theta \cos \theta+\rho^{2} \alpha \operatorname{sen} \theta\right] \\
-\mu \rho^{3} \cos \theta-A \rho^{3}\left[\left(a_{1} \cos \omega s+a_{2} \operatorname{sen} \omega s\right) \cos \theta+\left(b_{1} \cos \omega s+b_{2} \operatorname{sen} \omega s\right) \operatorname{sen} \theta\right]
\end{array}
$$


Chamando $F(\theta, \rho)=\left(F_{1}(\theta, \rho), F_{2}(\theta, \rho)\right)$, com

$$
\begin{gathered}
F_{1}(\theta, \rho)=a \cos ^{3} \theta+(c-1) \operatorname{sen}^{2} \theta \cos \theta+\rho\left(m \cos ^{2} \theta+\operatorname{sen}^{2} \theta\right)+\rho^{2} \alpha \cos \theta, \\
F_{2}(\theta, \rho)=-\rho\left[\operatorname{csen}^{3} \theta+(a+1) \cos ^{2} \theta \operatorname{sen} \theta+\rho(m-1) \operatorname{sen} \theta \cos \theta+\rho^{2} \alpha \operatorname{sen} \theta\right], \\
G_{1}(\theta, \rho)=\left(-\rho^{2} \operatorname{sen} \theta,-\rho^{3} \cos \theta\right),
\end{gathered}
$$

$\mathrm{e}$

$$
G_{2}(\theta, \rho)=\left(G_{2}^{1}(\theta, \rho), G_{2}^{2}(\theta, \rho)\right)
$$

onde

$$
\begin{gathered}
G_{2}^{1}=\rho^{2}\left[\left(b_{1} \cos \omega s+b_{2} \operatorname{sen} \omega s\right) \cos \theta-\left(a_{1} \cos \omega s+a_{2} \operatorname{sen} \omega s\right) \operatorname{sen} \theta\right] \\
G_{2}^{2}=-\rho^{3}\left[\left(a_{1} \cos \omega s+a_{2} \operatorname{sen} \omega s\right) \cos \theta+\left(b_{1} \cos \omega s+b_{2} \operatorname{sen} \omega s\right) \operatorname{sen} \theta\right],
\end{gathered}
$$

pode-se escrever o sistema acima na forma

$$
\dot{u}=F(u)+\mu G_{1}(u)+A G_{2}\left(u \cdot \omega s+\phi_{0}\right),
$$

onde $u=(\theta, \rho), \phi(s)=\omega s+\phi_{0}$ é a solução de $\dot{\phi}=\omega, \operatorname{com} \phi(0)=\phi_{0}$.

Estamos assim, nas condiçōes apropriadas para aplicar os resultados da seção anterior. Vamos mostrar que são verdadeiras as hipóteses do Teorema 4.2.4 para a perturbação periódica acima, ou seja, que as funçòes $S_{m}$ e $M_{m}\left(\phi_{0}\right)$ associadas ao sistema (4.31), definidas no enunciado do referido Teorema. são tais que $S_{m} \neq 0$ e $M_{m}\left(\phi_{0}\right)$ possui um ponto de máximo, $\phi_{\max }$, e um ponto de mínimo, $\phi_{\min }$, nào degenerados, com $Y^{\prime \prime}\left(\phi_{\max }\right) \neq 0$ e $M^{\prime \prime}\left(\phi_{\min }\right) \neq 0$.

Primeiramente, provemos o seguinte Lema, que dá as expressòes das funçòes $S_{m}$ e $M_{m}\left(\phi_{0}\right)$, que aparecem no enunciado do Teorema 4.2.4, para o sistema (4.31).

Lema 4.3.2 As funções $S_{m} \in M_{m}\left(O_{0}\right)$. definidas no enunciado do Teorema 4.2.4, relativas ao sistema (4.31), são dadas por

$$
S_{m}=-\int_{0}^{m T} e^{-\int_{0}^{s} \sigma(\tau) d \tau} \rho^{3}\left[a \cos ^{2} \theta+\operatorname{csen}^{2} \theta+\rho m \cos \theta+\rho^{2} \alpha\right] d s
$$




$$
M_{m}\left(\phi_{0}\right)=-\int_{0}^{m T} e^{\left.-\int_{0}^{s} \sigma(\tau)\right) d \tau} \rho(s)^{3} K\left(\theta, \rho, \phi_{0}, s\right) d s,
$$

com

$$
\begin{aligned}
K\left(\theta, \rho, \phi_{0}, s\right)=\left[a_{1} \cos (\omega s\right. & \left.\left.+\phi_{0}\right)+a_{2} \operatorname{sen}\left(\omega s+\phi_{0}\right)\right]\left(a \cos ^{2} \theta+\operatorname{csen}^{2} \theta+\rho m \cos \theta+\rho^{2} \alpha\right) \\
& +\left[b_{1} \cos \left(\omega s+\phi_{0}\right)+b_{2} \operatorname{sen}\left(\omega s+\phi_{0}\right)\right]\left(\rho \operatorname{sen} \theta-\rho^{2} \operatorname{sen} \theta \cos \theta\right),
\end{aligned}
$$

onde $(\theta, \rho)=(\theta(s), \rho(s))$ são as coordenadas da órbita periódica ressonante $q^{\rho_{m}}(s)$ de (4.31), $e$

$$
\sigma(s)=\sum_{i=0}^{2} \rho(s)^{i} A_{n-i}^{\prime}(\theta(s))-\sum_{i=0}^{2}(i+1) \rho(s)^{i} R_{n-i}(\theta(s)),
$$

$\operatorname{com} A_{k}(\theta)$ e $R_{k}(\theta)$ definidas acima.

Prova: Considere o campo polinomial

$$
\begin{aligned}
& \dot{x}=P(x, y)+\epsilon f(\tilde{\omega} s) \\
& \dot{y}=Q(x, y)+\epsilon g(\tilde{\omega} s),
\end{aligned}
$$

e sua compactificação em coordenadas $(\theta, \rho)$ dada por

$$
\begin{aligned}
& \dot{\theta}=\sum_{i=1}^{n} \rho^{i} A_{n-i}(\theta)+\bar{f}(\theta, \rho, \epsilon, \phi) \\
& \dot{\rho}=-\sum_{i=0}^{n} \rho^{i+1} R_{n-i}(\theta)+\bar{g}(\theta, \rho, \epsilon, \phi) \\
& \dot{\phi}=\omega,
\end{aligned}
$$

onde

$$
\begin{gathered}
A_{k}(\theta)=-P_{k}(\cos \theta, \operatorname{sen} \theta) \operatorname{sen} \theta+Q_{k}(\cos \theta, \operatorname{sen} \theta) \cos \theta \\
R_{k}(\theta)=P_{k}(\cos \theta, \operatorname{sen} \theta) \cos \theta+Q_{k}(\cos \theta \cdot \operatorname{sen} \theta) \operatorname{sen} \theta,
\end{gathered}
$$

para $k=0,1, \ldots, n, \operatorname{com} \theta \in[0,2 \pi)$ e $\rho \in[0, \infty)$,

$$
\vec{f}(\theta, \rho, \epsilon, \phi)=\rho^{n}[\epsilon g(\phi) \cos \theta-\epsilon f(o) \operatorname{sen} \theta],
$$




$$
\bar{g}(\theta, \rho, \epsilon, \phi)=-\rho^{n+1}[\epsilon f(\phi) \cos \theta+\epsilon g(\phi) \operatorname{sen} \theta]
$$

Calculemos, para este sistema compactificado, os termos que comparecem no integrando da funçào $M\left(\phi_{0}\right)$, dada no enunciado do Teorema 4.2.4. Para tanto, chamemos

$$
F(\theta, \rho)=\left(\sum_{i=1}^{n} \rho^{i} A_{n-i}(\theta),-\sum_{i=0}^{n} \rho^{i+1} R_{n-i}(\theta)\right) .
$$

Assim, omitindo, por um momento, os argumentos das funções, e chamando $s=\operatorname{sen} \theta$ e $c=\cos \theta$, obtemos:

$$
\begin{gathered}
<F^{\perp},(\bar{f}, \bar{g})>= \\
<\left(c \sum_{i=0}^{n} \rho^{i+1} P_{n-i}+s \sum_{i=0}^{n} \rho^{i+1} Q_{n-i} \cdot-s \sum_{i=0}^{n} \rho^{i} P_{n-i}+c \sum_{i=0}^{n} \rho^{i} Q_{n-i}\right),(\bar{f}, \bar{g})>= \\
c \bar{f} \sum_{i=0}^{n} \rho^{i+1} P_{n-i}+s \bar{f} \sum_{i=0}^{n} \rho^{i+1} Q_{n-i}-s \bar{g} \sum_{i=0}^{n} \rho^{i} P_{n-i}+c \bar{g} \sum_{i=0}^{n} \rho^{i} Q_{n-i} .
\end{gathered}
$$

Substituindo as expressões de $\bar{f}$ e $\bar{g}$ na expressão acima, obtem-se, após alguns cálculos simples, envolvendo propriedades das funções trigonométricas, a igualdade

$$
\begin{aligned}
<F^{\perp},(\bar{f}, \bar{g})> & =g \rho^{n+1} \sum_{i=0}^{n} \rho^{i} P_{n-i}-f \rho^{n+1} \sum_{i=0}^{n} \rho^{i} Q_{n-i} \\
& =\rho^{n+1}<\left(-\sum_{i=0}^{n} \rho^{i} Q_{n-i}, \sum_{i=0}^{n} \rho^{i} P_{n-i}\right),(f, g)>,
\end{aligned}
$$

onde $P_{k}$ e $Q_{k}$ são polinômios homogêneos de grau $k$, componentes dos polinômios $P$ e $Q$ que determinam o campo não perturbado no plano, isto é, $P=\sum_{k=0}^{n} P_{k}$ e $Q=\sum_{k=0}^{n} Q_{k}$, $f$ e $g$ são as funçòes de perturbação do campo polinomial $(P, Q)$, no plano, e $<$, > denota o produto interno usual do $\mathbb{R}^{2}$.

Para o sistema polinomial quadrático perturbado (4.29)-(4.30). cuja compactificação é dada por (4.31), existem dois pares $(f . g)$ de funçòes de perturbaçào, a saber: o par $(f, g)=(1,0)$, relativo ao parâmetro $\mu$ (perturbação autônoma), e o par

$$
(f, g)=\left(a_{1} \cos \omega s+a_{2} \operatorname{sen} \omega s, b_{1} \cos \omega s+b_{2} \operatorname{sen} \omega s\right)
$$

relativo ao parâmetro $A$ (perturbação periódica).

Substituindo os polinômios quadráticos que definem o sistema (4.29)-(4.30) e os pares de funções de perturbação acima na expressão de $\left\langle F^{\perp},(\bar{f}, \bar{g})\right\rangle$ obtida. e tendo 
em conta que o divergente do sistema quadrático compactificado não-perturbado, é dado por

$$
\operatorname{div} F\left(q^{\rho_{m}}(s)\right)=\sigma(s)=\sum_{i=0}^{n} \rho(s)^{i} A_{n-i}^{\prime}(\theta(s))-\sum_{i=0}^{n}(i+1) \rho(s)^{i} R_{n-i}(\theta(s))
$$

obtem-se as expressões de $S_{m}$ e $M_{m}\left(\phi_{0}\right)$.

Observação 4.3.3 Observe que $S_{m}$ independe de $\phi_{0}$ (pois está relacionada com a parte autônoma da perturbação).

Com o lema seguinte prova-se que $S_{m}<0$.

Lema 4.3.4 Para $\rho_{m}$ suficientemente pequeno, tem-se que

$$
\int_{0}^{m T} e^{-\int_{0}^{s} \sigma(\tau) d \tau} \rho(s)^{3}\left[a \cos ^{2} \theta(s)+c \operatorname{sen}^{2} \theta(s)+\rho(s) m \cos \theta(s)+\rho(s)^{2} \alpha\right] d s>0 .
$$

Prova: Considere a órbita periódica $q^{\rho_{m}}(s)=(\theta(s), \rho(s))$. Para $\rho_{m}$ suficientemente pequeno, tal órbita está próxima do ciclo singular infinito $\theta_{1} \cup \gamma(s) \cup \theta_{2} \cup \alpha(s)$ e podemos tomar as seções $\Sigma_{1}$ e $\Sigma_{2}$, transversais a esta órbita, definidas por

$$
\begin{gathered}
\Sigma_{1}=\left\{(\theta, \rho) \mid \theta \in\left(\frac{3 \pi}{2}-\epsilon, \frac{3 \pi}{2}+\epsilon\right) \quad \epsilon \quad \rho=\epsilon\right\} . \\
\Sigma_{2}=\left\{(\theta, \rho) \mid \theta \in\left(\frac{\pi}{2}-\epsilon, \frac{\pi}{2}+\epsilon\right) \quad \epsilon \quad \rho=\epsilon\right\} .
\end{gathered}
$$

com $\epsilon>0$ suficientemente pequeno (Figura 4.8).

Desse modo, supondo $q^{\rho_{m}}(0) \in \Sigma_{1}$, podemos dividir a integral em duas partes

$$
\begin{aligned}
& \int_{0}^{m T} e^{-\int_{0}^{s} \sigma(\tau) d \tau} \rho(s)^{3}\left[\operatorname{acos}^{2} \theta(s)+\operatorname{csen}^{2} \theta(s)+\rho(s) m \cos \theta(s)+\rho(s)^{2} \alpha\right] d s= \\
& \int_{0}^{T_{1}} e^{\left.-\int_{0}^{s} \sigma(\tau)\right) d \tau} \rho(s)^{3}\left[\operatorname{acos}^{2} \theta(s)+\operatorname{csen}^{2} \theta(s)+\rho(s) m \cos \theta(s)+\rho(s)^{2} \alpha\right] d s+ \\
& \int_{T_{1}}^{m T} e^{\left.-\int_{0}^{s} \sigma(\tau)\right) d \tau} \rho(s)^{3}\left[\operatorname{acos}^{2} \theta(s)+\operatorname{csen}^{2} \theta(s)+\rho(s) m \cos \theta(s)+\rho(s)^{2} \alpha\right] d s=S_{1}+S_{2},
\end{aligned}
$$




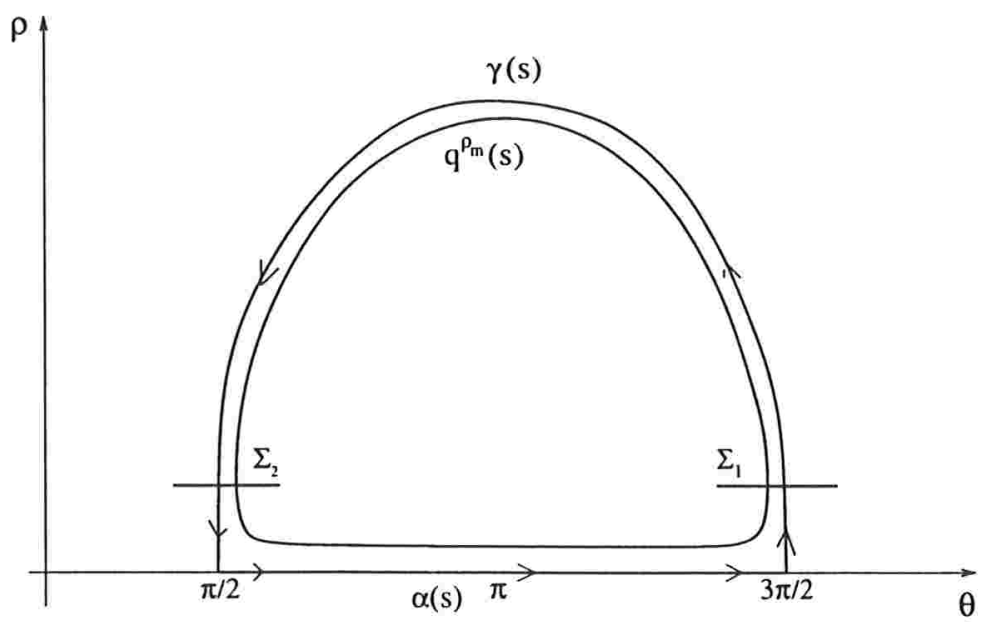

Figura 4.8: Orbita periódica $q^{\rho_{m}}(s)$, vista no plano $(\theta, \rho), \operatorname{com} \theta \in[0,2 \pi)$ e $\rho \in \mathbb{R}$, seccionada pelas seções transversais $\Sigma_{i}, i=1,2$. O eixo $\{\rho=0\}$ corresponde ao bordo do disco de Poincaré.

onde $T_{1}$ é o tempo que leva para a órbita $q^{\rho_{m}}(s)$ ir de $\Sigma_{1}$ até $\Sigma_{2}$, no sentido positivo.

Mostremos que a primeira das integrais na soma acima, $S_{1}$, é estritamente positiva e o valor da segunda integral, $S_{2}$, é muito pequeno, próximo de zero, para $\rho_{m}$ suficientemente pequeno.

Analisemos primeiramente $S_{2}$. Pela forma como foram tomadas as seções transversais acima (Figura 4.8) e como $q^{\rho_{m}}(s)$ fica $\epsilon$-próxima do ciclo singular $\gamma(s) \cup \theta_{1} \cup \alpha(s) \cup \theta_{2}$, para $s \in\left[T_{1}, m T\right]$ tem-se $0<\rho(s) \leq \epsilon<<1$, donde $\rho(s)^{3}<\epsilon$. Por outro lado, sendo $\rho(s)$ limitado, o termo

$$
\sigma(s)=\sum_{i=0}^{n} \rho(s)^{i} A_{n-i}^{\prime}(\theta(s))-\sum_{i=0}^{n}(i+1) \rho(s)^{i} R_{n-i}(\theta(s))
$$

é limitado, donde segue que $\exp \left(-\int_{0}^{s} \sigma(\tau) d \tau\right)$ é limitado para $s \in\left[T_{1}, m T\right]$, digamos por uma constante $K_{1}<\infty$. Além disso, é claro que $\left[a \cos ^{2} \theta(s)+\operatorname{csen}^{2} \theta(s)+\rho(s) m \cos \theta(s)+\right.$ $\left.\rho(s)^{2} \alpha\right] \leq K_{2}$, para uma constante $K_{2}<\infty$. Então tem-se

$$
\left|\int_{T_{1}}^{m T} e^{\left.-\int_{0}^{s} \sigma(\tau)\right) d \tau} \rho(s)^{3}\left[\cos ^{2} \theta(s)+\operatorname{csen}^{2} \theta(s)+\rho(s) m \cos \theta(s)+\rho(s)^{2} \alpha\right] d s\right|<\kappa_{1} K_{2}\left(m T-T_{1}\right) \epsilon .
$$


Para mostrar que a integral $S_{1}$ é estritamente positiva, usa-se o fato de que, para $\rho_{m}$ suficientemente pequeno e $s \in\left[0, T_{1}\right]$, tem-se, pelo Teorema da Dependência Contínua. que $q^{\rho_{m}}(s)$ fica próxima da porção da órbita heteroclínica $\gamma(s)$ compreendida entre as seções transversais $\Sigma_{1}$ e $\Sigma_{2}$ (Figura 4.8). Considerando as relações

$$
x \rho=\cos \theta, \quad y \rho=\operatorname{sen} \theta \quad \epsilon \quad \frac{d s}{d t}=\frac{1}{\rho_{n-1}}
$$

(conforme observação 2.2.2, Capítulo 2), e fazendo esta mudança de variáveis na integral acima obtem-se

$$
\begin{array}{r}
S_{1}=\int_{0}^{T_{1}} e^{\left.-\int_{0}^{s} \sigma(\tau)\right) d \tau} \rho(s)^{3}\left[a \cos ^{2} \theta(s)+\operatorname{csen}^{2} \theta(s)+\rho(s) m \cos \theta(s)+\rho(s)^{2} \alpha\right] d s= \\
\int_{t_{1}}^{t_{2}} \epsilon^{\left.-\int_{0}^{s} \tilde{\sigma}(\tau)\right) d \tau}\|(x(t), y(t))\|^{-2}\left[a x(t)^{2}+c y(t)^{2}+m x(t)+\alpha\right] d t= \\
\int_{t_{1}}^{t_{2}} e^{\left.-\int_{0}^{s} \tilde{\sigma}(\tau)\right) d \tau}\|(x(t), y(t))\|^{-2} Q(x(t), y(t)) d t,
\end{array}
$$

onde $q(t)=(x(t), y(t))$, com $t \in\left[t_{1}, t_{2}\right]$, é a porção da órbita periódica contida no plano, em coordenadas $(x, y)$, que é uma reparametrização da porçào da órbita periódica $q^{\rho_{m}}(s)$, com $s \in\left[0, T_{1}\right]$. sendo que a relação entre os tempos $0, T_{1}$, no disco de Poincaré. e $t_{1}, t_{2}$, no plano-( $\left.x, y\right)$, é dada pelo Lema 2.2 .3 (Capítulo 2 ).

Como a mudança de coordenadas (4.34) é um difeomorfismo para $(x, y) \neq(0,0)$, e como, para $s \in\left[0, T_{1}\right] \cdot q^{\rho_{m}}(s)$ fica $\epsilon$-próxima de $\hat{i}_{i}(s)$. no disco de Poincaré, segue do Teorema da Dependência Contínua, que para $t \in\left[t_{1}, t_{2}\right]$ a órbita periódica no plano. $q(t)=(x(t), y(t))$, fica próxima da solução $\varphi(t)=\left(1 . \varphi_{2}(t)\right)$ do sistema polinomial no plano, contida na reta invariante $\{x=1\}$, correspondente a $\gamma(s)$ (conforme a Proposiçào 3.1.1). Assim, a coordenada $x(t)$ da órbita periódica $q(t)$ fica próxima de 1 , para $t \in\left[t_{1}, t_{2}\right]$. Como, por hipótese, $Q(1, y)>0, \forall y$. tem-se, por continuidade, que o integrando de $S_{1}$ é produto de funções positivas e, portanto, a integral é estritamente positiva.

Portanto, tomando-se $\epsilon$ suficientemente pequeno na construção das seções transversais $\Sigma_{1}$ e $\Sigma_{2}$, fica provado o Lema. 
Do Lema acima segue que $S_{m}<0$.

Lema 4.3.5 A função $M_{m}\left(\phi_{0}\right)$, associada ao sistema (4.31), possui dois únicos pontos críticos nâo degenerados, no intervalo $[0,2 \pi)$, sendo um de máximo e um de mínimo.

Prova: Pelo Lema (4.3.2), a expressão de $M_{m}\left(\phi_{0}\right)$ é dada por

$$
M_{m}\left(\phi_{0}\right)=\int_{0}^{m T} e^{-\int_{0}^{s} \sigma(\tau) d \tau} \rho(s)^{3} K\left(\theta, \rho, \phi_{0}\right) d s,
$$

onde

$$
\sigma(s)=\sum_{i=0}^{n} \rho^{i} A_{n-i}^{\prime}(\theta)-\sum_{i=0}^{n}(i+1) \rho^{i} R_{n-i}(\theta),
$$

que independe de $\phi_{0}$, e

$$
\begin{array}{r}
K\left(\theta, \rho, \phi_{0}\right)=\left[a_{1} \cos \left(\omega s+\phi_{0}\right)+a_{2} \operatorname{sen}\left(\omega s+\phi_{0}\right)\right]\left(a \cos ^{2} \theta+\operatorname{csen}^{2} \theta+\rho m \cos \theta+\rho^{2} \alpha\right) \\
+\left[b_{1} \cos \left(\omega s+\phi_{0}\right)+b_{2} \operatorname{sen}\left(\omega s+\phi_{0}\right)\right]\left(\rho \operatorname{sen} \theta-\rho^{2} \operatorname{sen} \theta \cos \theta\right),
\end{array}
$$

onde $(\theta, \rho)=(\theta(s), \rho(s))$ são as coordenadas da órbita periódica ressonante $q^{\rho_{m}}(s)$ de (4.31).

Derivando então $M_{m}\left(\phi_{0}\right)$ com relação a $\phi_{0}$ segue que $M_{m}^{\prime \prime}\left(\phi_{0}\right)=-M_{m}\left(\phi_{0}\right)$, para todo $\phi_{0} \in[0,2 \pi)$. Assim, $M\left(\phi_{0}\right)$ é solução da equação diferencial ordinária $x^{\prime \prime}+x=0$ e, portanto, tem a forma

$$
M_{m}\left(\phi_{0}\right)=B \cos \left(\phi_{0}\right)+D \operatorname{sen}\left(\phi_{0}\right)
$$

onde as constantes $B$ e $D$ dependem da condição inicial considerada. Portanto, $M_{m}\left(\phi_{0}\right)$ possui um ponto de máximo e um ponto de mínimo, no intervalo $[0,2 \pi)$, não-degenerados, $\operatorname{com} M\left(\phi_{\min }\right)<0<M\left(\phi_{\max }\right)$.

Dos Lemas 4.3.4 e 4.3.5 pode-se concluir, usando o Teorema 4.2.4, que o diagrama de bifurcação do sistema 4.31 é como o mostrado na Figura 4.9. 


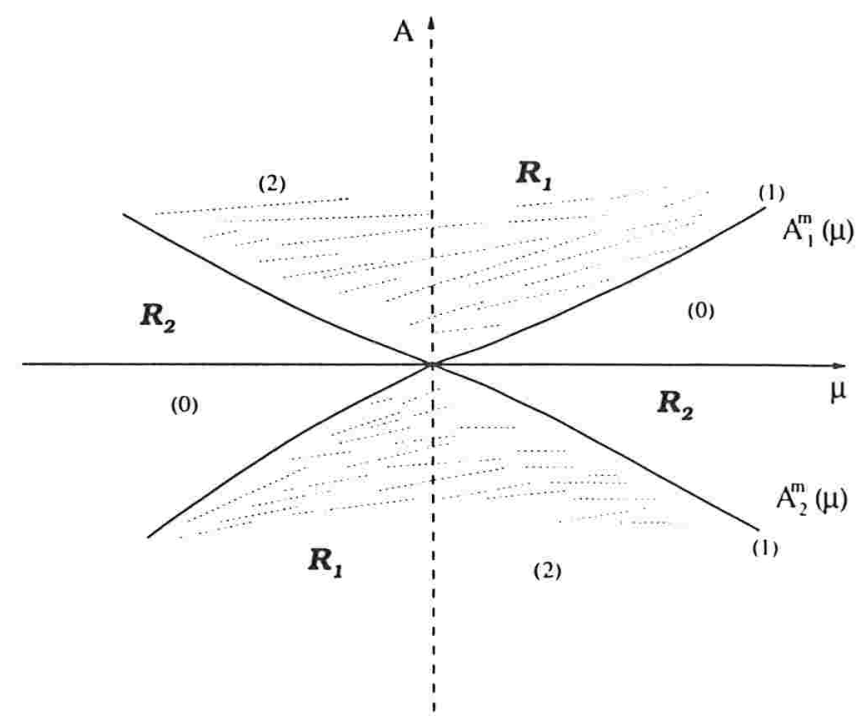

Figura 4.9: Diagrama de bifurcação do sistema quadrático. Os números entre parentesis se referem a quantidade de subharmonicas de ordem $m$ do sistema, que bifurcam de $q^{\rho_{m}}(s)$, para os respectivos valores dos parámetros.

Observe que, como neste caso $S_{m}<0$, as curvas de bifurcação de subharmônicas, $A_{1}^{m}(\mu)$ e $A_{2}^{m}(\mu)$, aparecem em posições trocadas, com relação à Figura 4.6, relativa ao Teorema 4.2.4. onde foi considerado $S_{m}>0$.

Concluindo. existem regiões no espaço de parâmetros, $(\mu, \mathcal{A})$. para as quais o sistema (4.31) possui órbitas periódicas subharmônicas de ordem $m$, bifurcando da órbita periódica ressonante $q^{\rho_{m}}(s)$ e regiòes onde não bifurcam subharmônicas de $q^{\rho_{n}}(s)$. Tais regiões são determinadas por curvas que dependem dos valores críticos, de máximo e mínimo, de $M_{m}\left(\phi_{0}\right)$. que, por sua vez, dependem diretamente das funções periódicas de perturbação do sistema, $f\left(\omega s+\phi_{0}\right)$ e $g\left(\omega s+\phi_{0}\right)$. 


\subsection{Comportamento assintótico das bifurcações sub- harmônicas - caso quadrático}

As órbitas subharmônicas de ordem $m$, detectadas com o Teorema da seção anterior, correspondem somente àquelas que bifurcam da órbita ressonante $q^{\rho_{m}}(s)$, quando variamos os parâmetros $\mu$ e $A$ numa vizinhança da origem, no espaço de parâmtros.

Pelo Lema 4.2.2, para $\bar{\rho}$ suficientemente pequeno, existe uma sequência enumerável $\rho_{m_{i}} \subset(0, \bar{\rho})$, com $i \in \mathbb{N}$, tal que, a cada elemento da sequência, corresponde uma órbita periódica ressonante $q^{\rho_{m_{i}}}(s)$, do sistema (4.31), contida no anel regular de órbitas periódicas que se acumula no ciclo singular infinito. Além disso, $\lim _{i \rightarrow+\infty} \rho_{m_{i}}=0$, donde se conclui que as órbitas periódicas ressonantes $q^{\rho_{m_{i}}}(s)$ tendem, como conjunto, para o ciclo singular infinito, quando $i \rightarrow+\infty$.

Com base nestas considerações, será feita, nesta seção, uma análise do comportamento limite das órbitas periódicas subharmônicas de ordem $m_{i}$, que bifurcam das órbitas ressonantes $q^{\rho_{m_{i}}}(s)$, quando a sequência $\rho_{m_{\mathrm{t}}}$ tende a zero, para a família de campos quadráticos com perturbação periódica, (4.31), da seção anterior.

Mostraremos que as inclinações das retas tangentes, na origem, às curvas de bifurcações subharmônicas, $A_{1}^{m_{i}}(\mu)$ e $A_{2}^{m_{i}}(\mu)$, associadas a órbita ressonante $q^{\rho_{m_{i}}}(s)$, temdem, quando $i \rightarrow+\infty$, para as inclinações das retas tangentes às curvas de tangências heteroclínicas

$$
A_{M}(\mu)=-\frac{S}{M\left(\phi_{\max }\right)} \mu-O\left(\mu^{2}\right)
$$

$\mathrm{e}$

$$
A_{m}(\mu)=-\frac{S}{M\left(\phi_{\min }\right)} \mu-O\left(\mu^{2}\right)
$$

obtidas no Capítulo 3. Em outras palavras, mostraremos as seguintes convergências

$$
S_{m_{i}} \rightarrow S, \quad M_{m_{i}}\left(\phi_{\max }\right) \rightarrow M\left(\phi_{\max }\right) \quad \text { e } \quad M_{m_{i}}\left(\phi_{\min }\right) \rightarrow M\left(\phi_{\min }\right),
$$

para $i \rightarrow+\infty$. 
Como, para cada $m_{i}$ existem duas subharmônicas de ordem $m_{i}$, que bifurcam das órbitas ressonantes $q^{\rho_{m_{1}}}(s)$, quando os parâmetros $\mu, A$ cruzam as curvas $A_{1}^{m_{i}}(\mu) \mathrm{e}$ $A_{2}^{m_{i}}(\mu)$, então, se $m_{i} \rightarrow+\infty$, o número total de subharmônicas do sistema tende também para infinito. Por outro lado, como as órbitas ressonantes $q^{\rho_{m_{1}}}(s)$ se acumulam no ciclo singular infinito $\gamma(s) \cup \theta_{1} \cup \alpha(s) \cup \theta_{2}$, as subharmônicas de ordem $m_{i}$ que bifurcam delas se acumulam nas tangências heteroclínicas, desde que as convergências acima sejam verificadas. Na Figura 4.10 é mostrado um esquema pictórico, com base nas considerações acima.

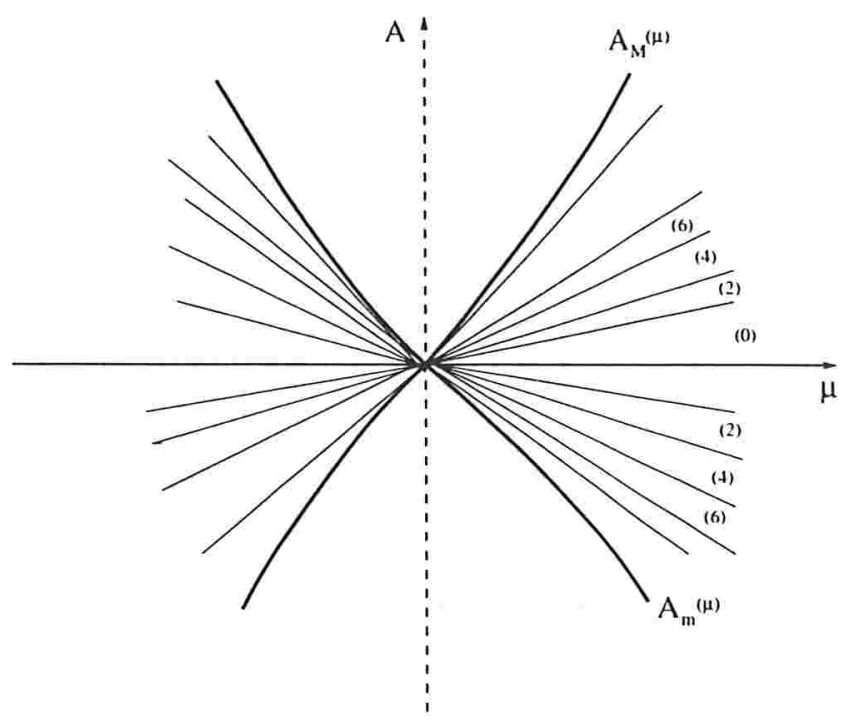

Figura 4.10: As inclinaçōes das curvas de bifurcaçōes subharmonicas, $A_{1}^{m_{1}}(\mu)$ e $A_{2}^{m_{1}}(\mu)$, associadas a órbita ressonante $q^{\rho_{m}}(s)$. tendem, quando $i \rightarrow+\infty$ (e, consequentemente $\left.m_{i} \rightarrow+\infty\right)$, para as inclinações das curvas de tangéncias heteroclínicas $A_{M}(\mu)$ e $A_{m}(\mu)$, obtidas no Capítulo 3 . O número entre parentesis indica a quantidade mínima de subharmonicas do sistema para $(\mu, A)$ pertencente aquela região do espaço de parámetros.

Considere então a seguinte perturbação periódica do sistema quadrático com dois centros e uma reta invariante

$$
\dot{x}=x y-y+\mu+A\left(a_{1} \cos (\tilde{\omega} t)+a_{2} \operatorname{sen}(\tilde{\omega} t)\right)
$$




$$
\dot{y}=\alpha+m x+a x^{2}+c y^{2}+A\left(b_{1} \cos (\tilde{\omega} t)+b_{2} \operatorname{sen}(\tilde{\omega} t)\right),
$$

conforme a seção anterior. Sua compactificação no disco de Poincaré fornece o sistema

$$
\dot{u}=F(u)+\mu G_{1}(u)+A G_{2}\left(u \cdot \omega s+\phi_{0}\right),
$$

definido em (4.31).

Conforme visto na seção anterior, as curvas de bifurcações subharmônicas do sistema acima, associadas a uma órbita periódica ressonante $q^{\rho_{m_{i}}}(s)$, são dadas por

$$
A_{1}^{m_{i}}(\mu)=-\frac{S_{m_{i}}}{M_{m_{i}}\left(\phi_{\max }\right)} \mu-O\left(\mu^{2}\right)
$$

e

$$
A_{2}^{m_{i}}(\mu)=-\frac{S_{m_{i}}}{M_{m_{1}}\left(\phi_{\min }\right)} \mu-O\left(\mu^{2}\right)
$$

onde

$$
\begin{gathered}
S_{m_{i}}=\int_{0}^{m_{i} T} e^{-\int_{0}^{s} \sigma(\tau) d \tau} \rho_{m_{i}}(s)^{3}\left[\operatorname{acos}^{2} \theta_{m_{i}}(s)+\operatorname{csen}^{2} \theta_{m_{1}}(s)+\rho_{m_{\mathrm{t}}}(s) m \cos \theta_{m_{\mathrm{t}}}(s)+\rho_{m_{i}}(s)^{2} \alpha\right] d s \\
M_{m_{i}}\left(\phi_{\max }\right)=\int_{0}^{m_{i} T} e^{-\int_{0}^{s} \sigma(\tau) d \tau} \rho_{m_{i}}(s)^{3} F\left(\theta_{m_{i}}(s), \rho_{m_{i}}(s), \phi_{\max }\right) d s, \\
M_{m_{i}}\left(\phi_{\min }\right)=\int_{0}^{m_{i} T} e^{-\int_{0}^{s} \sigma(\tau) d \tau} \rho_{m_{i}}(s)^{3} F\left(\theta_{m_{1}}(s), \rho_{m_{i}}(s), \phi_{\min }\right) d s,
\end{gathered}
$$

com

$$
\sigma(s)=\sum_{i=0}^{2} \rho_{m_{i}}(s)^{i} A_{n-i}^{\prime}\left(\theta_{m_{i}}(s)\right)-\sum_{i=0}^{2}(i+1) \rho_{m_{i}}(s)^{i} R_{n-i}\left(\theta_{m_{i}}(s)\right),
$$

e

$$
\begin{array}{r}
F\left(\theta_{m_{i}}(s), \rho_{m_{1}}(s), \phi_{0}\right)=-\left(a_{1} \cos \left(\omega s+\phi_{0}\right)+a_{2} \operatorname{sen}\left(\omega s+\phi_{0}\right)\right) \sum_{i=0}^{2} \rho_{m_{1}}(s)^{i} Q_{n-i}\left(\cos \theta_{m_{1}}(s), \operatorname{sen} \theta_{m_{1}}(s)\right)+ \\
\left(b_{1} \cos \left(\omega s+\phi_{0}\right)+b_{2} \operatorname{sen}\left(\omega s+\phi_{0}\right)\right) \sum_{i=0}^{2} \rho_{m_{1}}(s)^{i} P_{n-i}\left(\cos \theta_{m_{\mathrm{t}}}(s), \operatorname{sen} \theta_{m_{\mathrm{t}}}(s)\right),
\end{array}
$$

sendo $\left(\theta_{m_{i}}(s), \rho_{m_{i}}(s)\right)$ as coordenadas da órbita periódica ressonante $q^{\rho_{m_{i}}}(s), P_{k}$ e $Q_{k}$ os polinômios homogêneos de grau $k$ tais que $P(x, y)=\sum_{k=0}^{2} P_{k}(x, y)$ e $Q(x, y)=$ 
$\sum_{k=0}^{2} Q_{k}(x, y)$, onde $P(x, y)=x y-y$ e $Q(x, y)=\alpha+m x+a x^{2}+c y^{2}$ são os polinômios que determinam o sistema quadrático no plano.

Do estudo desenvolvido no Capítulo 3, segue que as tangências heteroclínicas, associadas à conexão heteroclínica $\gamma(s)$ do sistema (4.31), são determinadas, no espaço de parâmetros pelas curvas

$$
A_{M}(\mu)=-\frac{S}{M\left(\phi_{\max }\right)} \mu-O\left(\mu^{2}\right)
$$

$\mathrm{e}$

$$
A_{m}(\mu)=-\frac{S}{M\left(\phi_{\min }\right)} \mu-O\left(\mu^{2}\right)
$$

onde

$$
\begin{gathered}
S=\int_{-\infty}^{+\infty} e^{-\int_{0}^{s} \sigma(\tau) d \tau} \rho_{0}(s)^{3}\left[a \cos ^{2} \theta_{0}(s)+\operatorname{csen}^{2} \theta_{0}(s)+\rho m \cos \theta_{0}(s)+\rho^{2}(s) \alpha\right] d s, \\
M\left(\phi_{\min }\right)=\int_{-\infty}^{+\infty} e^{-\int_{0}^{s} \sigma(\tau) d \tau} \rho_{0}(s)^{3} F\left(\theta_{0}(s), \rho_{0}(s) \cdot \phi_{\min }\right) d s \\
M\left(\phi_{\max }\right)=\int_{-\infty}^{+\infty} e^{-\int_{0}^{s} \sigma(\tau) d \tau} \rho_{0}(s)^{3} F\left(\theta_{0}(s) \cdot \rho_{0}(s) \cdot \rho_{\max }\right) d s
\end{gathered}
$$

com

$$
\sigma(s)=\sum_{i=0}^{2} \rho_{0}(s)^{i} A_{n-i}^{\prime}\left(\theta_{0}(s)\right)-\sum_{i=0}^{2}(i+1) \rho_{0}(s)^{i} R_{n-i}\left(\theta_{0}(s)\right)
$$

$\mathrm{e}$

$$
\begin{array}{r}
F\left(\theta_{0}(s), \rho_{0}(s), \phi_{0}\right)=-\left(a_{1} \cos \left(\omega s+\phi_{0}\right)+a_{2} \operatorname{sen}\left(\omega s+\phi_{0}\right)\right) \sum_{i=0}^{n} \rho_{0}(s)^{i} Q_{n-i}\left(\cos \left(\theta_{0}(s)\right) \cdot \operatorname{sen}\left(\theta_{0}(s)\right)\right)+ \\
\left(b_{1} \cos \left(\omega s+\phi_{0}\right)+b_{2} \operatorname{sen}\left(\omega s+\phi_{0}\right)\right) \sum_{i=0}^{n} \rho_{0}(s)^{i} P_{n-i}\left(\cos \left(\theta_{0}(s)\right) \cdot \operatorname{sen}\left(\theta_{0}(s)\right)\right),
\end{array}
$$

sendo $\left(\theta_{0}(s), \rho_{0}(s)\right)$ as coordenadas da órbita heteroclínica $\gamma(s)$. que conecta os pontos de sela, $\theta_{1}$ e $\theta_{2}$, do campo compactificado não perturbado, e $P_{k}$ e $Q_{k}$ são os polinômios homogêneos de grau $k$ tais que $P(x, y)=\sum_{k=0}^{2} P_{k}(x, y)$ e $Q(x, y)=\sum_{k=0}^{2} Q_{k}(x, y)$, sendo $P$ e $Q$ os polinômios quadráticos que determinam o sistema no plano.

Observe a semelhança das integrais $S \operatorname{com} S_{m}$ e $M\left(\phi_{0}\right)$ com $M_{m}\left(\phi_{0}\right)$. Mostremos então, primeiramente. que $S_{m_{\imath}} \rightarrow S$, para $i \rightarrow+\infty$. 
Lema 4.4.1 $S_{m_{i}} \rightarrow S$, se $i \rightarrow+\infty$.

Prova: Para estabelecer a convergência acima, vamos mostrar que, para todo $\epsilon>0$ dado, existe $i \in \mathbb{N}$ tal que $\left|S-S_{m_{i}}\right|<\epsilon$.

Como $\rho_{m_{\mathrm{t}}} \rightarrow 0$, para $i \rightarrow+\infty$, então a órbita periódica ressonante correspondente, $q^{\rho_{m_{i}}}(s)$ tal que $q^{\rho_{m_{i}}}(0)=\rho_{m_{i}}$, tende, como conjunto, para o ciclo singular $\gamma(s) \cup \theta_{1} \cup \alpha \cup \theta_{2}$ (Figura 4.11), para $i \rightarrow+\infty$.

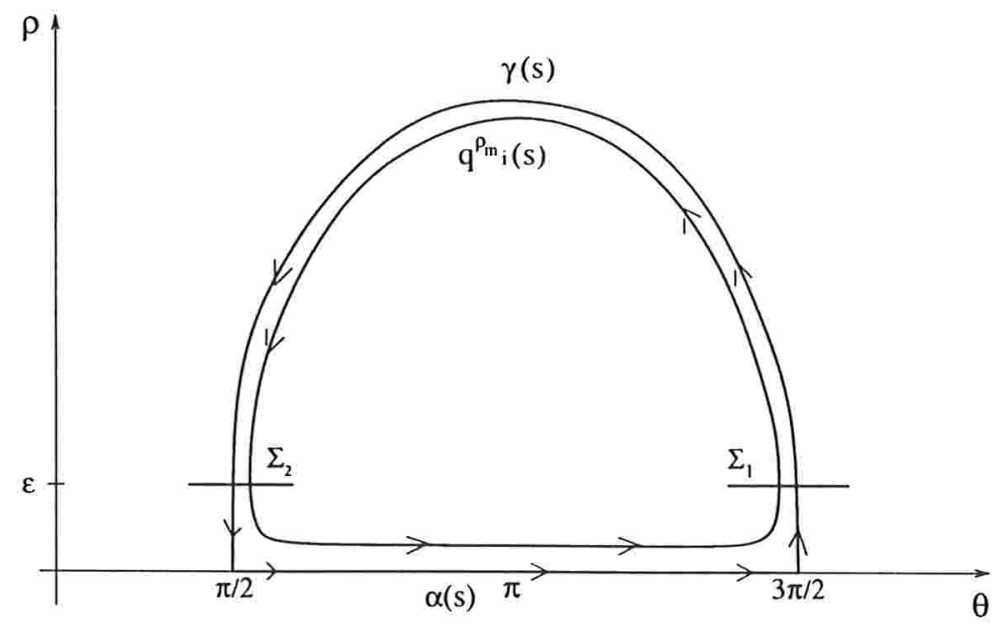

Figura 4.11: Orbita periódica $q^{\rho_{m}}(s)$, vista no plano $(\theta, \rho), \epsilon$-próxima do ciclo singular infinito $\gamma(s) \cup \theta_{1} \cup \alpha \cup \theta_{2}$, para $i$ suficientemente grande, seccionada pelas seçōes transversais $\Sigma_{i}, i=1,2$.

Assim podemos tomar as seções transversais a $q^{\rho_{m_{1}}}(s)$ e a $\gamma(s)$, definidas por

$$
\begin{gathered}
\Sigma_{1}=\left\{(\theta, \rho) \mid \theta \in\left(\frac{3 \pi}{2}-\epsilon, \frac{3 \pi}{2}+\epsilon\right) \quad \epsilon \quad \rho=\epsilon\right\}, \\
\Sigma_{2}=\left\{(\theta, \rho) \mid \theta \in\left(\frac{\pi}{2}-\epsilon, \frac{\pi}{2}+\epsilon\right) \quad \epsilon \quad \rho=\epsilon\right\},
\end{gathered}
$$

com $\epsilon>0$ suficientemente pequeno (Figura 4.11).

Como, para $i \rightarrow+\infty, q^{\rho_{m_{i}}}(s)$ tende, como conjunto, para o ciclo singular $\gamma(s) \cup \theta_{1} \cup$ $\alpha \cup \theta_{2}$, para $i$ suficientemente grande tem-se, pelo Teorema da Dependência Contínua, 
que existe $\tau>0$ tal que $q^{\rho_{m_{1}}}(s)$ fica $\epsilon$-próxima de $\gamma(s)$, para todo $s \in[-\tau, \tau]$, com (Figura 4.11)

$$
q^{\rho_{m_{i}}}(-\tau), \gamma(-\tau) \in \Sigma_{1} \quad \text { e } \quad q^{\rho_{m_{i}}}(\tau), \gamma(\tau) \in \Sigma_{2}
$$

Consideremos então a integral $S$ escrita na forma

$$
\begin{aligned}
S & =\int_{-\infty}^{-\tau} e^{-\int_{0}^{s} \sigma(\tau) d \tau} \rho_{0}(s)^{3}\left[\operatorname{acos}^{2} \theta_{0}(s)+\operatorname{csen}^{2} \theta_{0}(s)+\rho m \cos \theta_{0}(s)+\rho^{2}(s) \alpha\right] d s \\
& +\int_{-\tau}^{\tau} e^{-\int_{0}^{s} \sigma(\tau) d \tau} \rho_{0}(s)^{3}\left[\operatorname{acos}^{2} \theta_{0}(s)+\operatorname{csen}^{2} \theta_{0}(s)+\rho m \cos \theta_{0}(s)+\rho^{2}(s) \alpha\right] d s \\
& +\int_{\tau}^{+\infty} e^{-\int_{0}^{s} \sigma(\tau) d \tau} \rho_{0}(s)^{3}\left[a \cos ^{2} \theta_{0}(s)+\operatorname{csen}^{2} \theta_{0}(s)+\rho m \cos \theta_{0}(s)+\rho^{2}(s) \alpha\right] d s .
\end{aligned}
$$

Usando agora a periodicidade de $q^{\rho_{m_{i}}}(s)$, podemos escrever a integral $S_{m_{\mathrm{t}}}$ da seguinte maneira

$S_{m_{i}}=\int_{-m_{i} T / 2}^{m_{i} T / 2} e^{-\int_{0}^{s} \sigma(\tau) d \tau} \rho_{m_{i}}(s)^{3}\left[\operatorname{acos}^{2} \theta_{m_{i}}(s)+\operatorname{csen}^{2} \theta_{m_{i}}(s)+\rho_{m_{i}}(s) m \cos \theta_{m_{i}}(s)+\rho_{m_{i}}^{2}(s) \alpha\right] d s$

e, assim, como $m_{i} \rightarrow+\infty$, para $i \rightarrow+\infty$ (conforme Lema 4.2.2), podemos escrever

$$
\begin{aligned}
S_{m_{\mathrm{i}}} & =\int_{-m_{\mathrm{t}} T / 2}^{-\tau} \epsilon^{-\int_{0}^{s} \sigma(\tau) d \tau} \rho_{m_{i}}^{3}\left[a \cos ^{2} \theta_{m_{\mathrm{t}}}+\operatorname{csen}^{2} \theta_{m_{\mathrm{t}}}+\rho_{m_{\mathrm{t}}} m \cos \theta_{m_{\mathrm{t}}}+\rho_{m_{\mathrm{t}}}^{2} \alpha\right] d s \\
& +\int_{-\tau}^{\tau} e^{-\int_{0}^{s} \sigma(\tau) d \tau} \rho_{m_{\mathrm{t}}}^{3}\left[\operatorname{acos}^{2} \theta_{m_{i}}+\operatorname{csen}^{2} \theta_{m_{i}}+\rho_{m_{i}} m \cos \theta_{m_{\mathrm{t}}}+\rho_{m_{\mathrm{t}}}^{2} \alpha\right] d s \\
& +\int_{\tau}^{m_{\mathrm{i}} T / 2} \epsilon^{-\int_{0}^{s} \sigma(\tau) d \tau} \rho_{m_{i}}^{3}\left[\cos ^{2} \theta_{m_{i}}+\operatorname{csen}^{2} \theta_{m_{\mathrm{t}}}+\rho_{m_{\mathrm{t}}} m \cos \theta_{m_{\mathrm{t}}}+\rho_{m_{\mathrm{t}}}^{2} \alpha\right] d s
\end{aligned}
$$

onde $\theta_{m_{i}}=\theta_{m_{i}}(s)$ e $\rho_{m_{\mathrm{t}}}=\rho_{m_{i}}(s)$ são as coordenadas da órbita periódica ressonante

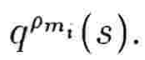

Para simplificar a notação nos cálculos que vem a seguir, chamemos $F\left(q^{\rho_{m_{l}}}(s)\right)$ e $G(\gamma(s))$ os integrandos de $S_{m_{1}}$ e $S$, respectivamente. Procedendo assim, das considerações acima tem-se que

$$
\begin{aligned}
\left|S-S_{m_{i}}\right| & =\mid \int_{-\infty}^{-\tau} G(\gamma(s)) d s+\int_{-\tau}^{\tau} G(\gamma(s)) d s+\int_{\tau}^{+\infty} G(\gamma(s)) d s \\
& -\int_{-m_{1} T / 2}^{-\tau} F\left(q^{\rho_{m_{i}}}(s)\right) d s-\int_{-\tau}^{\tau} F\left(q^{\rho_{m_{i}}}(s)\right) d s-\int_{\tau}^{m_{i} T / 2} F\left(q^{\rho_{m_{i}}}(s)\right) d s \mid \\
& \leq\left|\int_{-\infty}^{-\tau} G(\gamma(s)) d s\right|+\left|\int_{-m_{i} T / 2}^{-\tau} F\left(q^{\rho_{m_{i}}}(s)\right) d s\right| \\
& +\left|\int_{-\tau}^{\tau} G(\gamma(s)) d s\right|+\left|\int_{-\tau}^{\tau} F\left(q^{\rho_{m_{i}}}(s)\right) d s\right| \\
& +\left|\int_{-}^{+\infty} G(\gamma(s)) d s\right|+\left|\int_{\tau}^{m_{i} T / 2} F\left(q^{\rho_{m_{i}}}(s)\right) d s\right| .
\end{aligned}
$$


Façamos uma análise de cada uma das integrais acima, para $i \rightarrow+\infty$ (e, consequentemente, $\rho_{m_{i}} \rightarrow 0$ ).

Como, para $i$ suficientemente grande e $s \in[-\tau, \tau]$, temos que $\gamma(s)$ e $q^{\rho_{m_{i}}}(s)$ ficam $\epsilon$-próximas (Teorema da Dependência Contínua) e, sendo $G$ e $F$ funções contínuas de $\gamma(s)$ e $q^{\rho_{m_{i}}}(s)$, respectivamente, com

$$
\lim _{i \rightarrow+\infty} F\left(q^{\rho_{m_{i}}}(s)\right)=G_{i}^{\prime}(\gamma(s))
$$

tem-se que, para $i$ suficientemente grande,

$$
\left|\int_{-\tau}^{\tau} G(\gamma(s)) d s-\int_{-\tau}^{\tau} F\left(q^{\rho_{m_{i}}}(s)\right) d s\right|=\left|\int_{-\tau}^{\tau}\left[G(\gamma(s))-F\left(q^{\rho_{m_{i}}}(s)\right)\right] d s\right|<\epsilon .
$$

Seja $\gamma(s)=\left(\theta_{0}(s), \rho_{0}(s)\right)$ e $q^{\rho_{m_{i}}}(s)=\left(\theta_{m_{i}}(s), \rho_{m_{i}}(s)\right)$. Pela construção das seções transversais $\Sigma_{1}$ e $\Sigma_{2}$ (Figura 4.11) e pelo fato de $q^{\rho_{m_{i}}}(s)$ tender ao ciclo singular $\gamma(s) \cup$ $\theta_{1} \cup \alpha(s) \cup \theta_{2}$, segue que, para $i$ suficientemente grande e $s \in[-\infty,-\tau)$ ou $s \in[\tau,+\infty)$, valem as desigualdades

$$
\left|\theta_{m_{i}}(s)\right|<\epsilon \quad e \quad\left|\rho_{m_{i}}(s)\right|<\epsilon
$$

Então tem-se

$$
\left|\int_{-m_{i} T / 2}^{-\tau} F\left(q^{\rho_{m_{i}}}(s)\right) d s\right| \leq \int_{-m_{\mathrm{i}} T / 2}^{-\tau}\left|F\left(q^{\rho_{m_{i}}}(s)\right)\right| d s<\epsilon_{1} M_{1}
$$

e também

$$
\left|\int_{\tau}^{m_{i} T / 2} F\left(q^{\rho_{m_{i}}}(s)\right) d s\right| \leq \int_{\tau}^{m_{i} T / 2}\left|F\left(q^{\rho_{m_{i}}}(s)\right)\right| d s \quad<\epsilon_{2} M_{2}
$$

onde

$$
M_{1}=\sup _{s \in\left[-m_{i} T / 2,-\tau\right]}\left|F\left(q^{\rho_{m_{i}}}(s)\right)\right| \quad e \quad M_{2}=\sup _{s \in\left[\tau, m_{i} T / 2\right]}\left|F\left(q^{\rho_{m_{i}}}(s)\right)\right| .
$$

Como $m_{i} \rightarrow+\infty$, para $i \rightarrow+\infty$, então, como as integrais impróprias acima são convergentes, podemos tomar $\tau$ suficientemente grande (digamos $\tau=\frac{m_{i} T}{2}-\epsilon$ ), de maneira que

$$
\left|\int_{-\infty}^{-\tau} G(\gamma(s)) d s\right|<\epsilon / 4
$$


$\mathrm{e}$

$$
\left|\int_{\tau}^{\infty} G(\gamma(s)) d s\right|<\epsilon / 4
$$

Tomando $\epsilon_{i}, i=1,2$ de forma apropriada nas majorações acima, pode-se concluir que

$$
\left|S-S_{m_{1}}\right|<\epsilon
$$

para todo $\epsilon$ dado, com o que fica provada a convergência das integrais, para $i \rightarrow+\infty$.

Lema 4.4.2 $M_{m_{1}}^{(j)}\left(\phi_{\max }\right) \rightarrow M^{(j)}\left(\phi_{\max }\right)$, se $i \rightarrow+\infty$, para $j=0,1,2$, onde $(j)$ denota a j-ésima derivada de $M$ com relação a $\phi_{0}$.

Prova: A prova é feita de maneira inteiramente análoga à prova do Lema 4.4.1.

Com a prova dos lemas acima, fica estabelecido que as tangências heteroclínicas entre as variedades invariantes das órbitas periódicas no infinito, para o campo polinomial, são limites de bifurcações subharmônicas que ocorrem no anel de órbitas periódicas que se acumula no ciclo heteroclínico infinito. Além disso, como $m_{i} \rightarrow+\infty$, se $i \rightarrow+\infty$, e para cada $m_{i}$ existem duas subharmônicas de ordem $m_{i}$ que bifurcam da órbita periódica ressonante $q^{\rho_{m}}(s)$, pode-se concluir também que existem infintas subharmônicas do sistema, na vizinhança das tangências heteroclínicas.

Resultado semelhante foi obtido em [8], para outra classe de campos vetoriais com perturbação periódica. considerando-se regiòes compactas do plano. 


\section{Capítulo 5}

\section{Conclusões e Considerações Finais}

Podemos observar atualmente, na literatura relacionada com a área, a ocorrência de inúmeros sistemas determinísticos de equações diferenciais ordinárias, cujas soluções apresentam comportamento dito "caótico" (ver por exemplo, [1], [2], [11], [15], [22], [28], [29]).

Os exemplos mais conhecidos são as equações de Lorenz, equações de Duffing e do Pêndulo com termo forçante periódico, equações de Rösller, equações que determinam o circuito de Chua, e outras (conforme [1] ou [2]).

O complexo comportamento dinâmico das soluçōes destes sistemas é, em geral, atribuido ao fato deles apresentarem órbitas homoclínicas e/ou heteroclínicas a um ponto de equilíbrio ou a uma órbita periódica, contidos em regiões compactas do plano ou do espaço de fase.

No presente trabalho mostramos que se um campo polinomial, definido no plano todo, apresenta certas propriedades envolvendo o "infinito", entào certos tipos de perturbações periódicas deste campo podem levar a uma dinâmica bastante complexa de suas soluções.

A hipótese básica que colocamos sobre os sistemas polinomiais aqui estudados foi a da existência de uma solução ilimitada, que tende para infinito, em tempo finito. Com 
o artifício da compactificação de Poincaré, e a hipótese adicional de que tal solução ilimitada conecta dois pontos de sela "no infinito", pudemos provar que as soluções de tais sistemas polinomiais podem apresentar, para determinados valores dos parâmetros, uma dinâmica bastante complexa, devido à interseção transversal das variedades invariantes das órbitas periódicas no infinito. Tais intersecções ocorrem na parte "finita" do plano. São propriedades relacionadas com o "infinito" influenciando no comportamento das soluções na parte "finita" do plano.

As simulações numéricas desenvolvidas, e apresentadas no capítulo 3, sugerem que, se o sistema polinomial perturbado apresenta duas linhas de singularidades no infinito, suas soluções podem apresentar um comportamento dinâmico bastante complexo, e isso se dá numa vizinhança da origem no espaço de fase. Tal fato não é tão surpreendente. De fato, como o valor do campo polinomial é muito grande, para valores de $(x, y)$ grandes, é de se esperar que a perturbação periódica considerada (que é muito pequena com relação ao campo) influencie nas soluções do sistema somente numa vizinhança da origem. As simulações sugerem a existência de um atrator estranho para a Transformação de Poincaré determinada pelo fluxo do sistema no tempo $2 \pi$.

Embora os resultados teóricos obtidos sejam válidos para valores pequenos dos parâmetros (pertencentes a uma vizinhança da origem), as simulações numéricas foram efetuadas explorando-se o comportamento das soluçòes do sistema perturbado para valores grandes dos parâmetros. especialmente do parâmetro A. relativo à amplitude da perturbação periódica. Os resultados teóricos serviram como base e ponto de partida para o estudo numérico desenvolvido.

Outro fato sugerido pelas simulaçòes numéricas efetuadas, é que o suposto atrator, existente para a Transformação de Poincaré associada ao sistema do Exemplo 1 do Capítulo 2, não atrai somente os pontos de sua vizinhança, mas sim todos os pontos do plano, menos um conjunto de medida nula. Na Figura 5.1. fazemos. usando o disco de Poincaré, um esquema de como isso se dá: as trajetórias se afastam da regiào $\mathbf{R}$, próxima da origem, acompanhando a variedade estável do ponto de sela $\theta_{1}$ no infinito: chegando 
próximas a este ponto de sela, elas retornam pelo bordo do disco e são conduzidas de volta à região $\mathbf{R}$ pela variedade instável do outro ponto de sela no infinito, $\theta_{2}$; a única solução que "escapa" da região $\mathbf{R}$ e não retorna mais é a própria variedade estável de $\theta_{1}$.

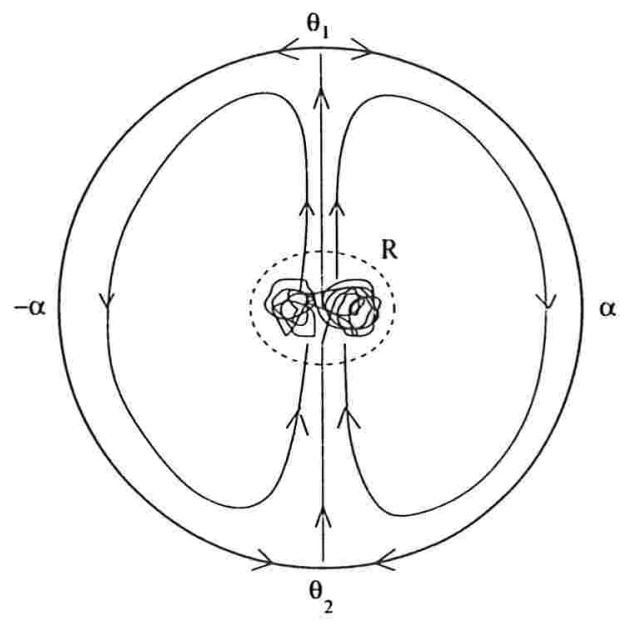

Figura 5.1: Esquema pictórico de como as iteradas da transformação de Poincaré associada ao campo polinomial perturbado do Exemplo 1 (Cap.2) tendem para uma vizinhança da origem (região $\mathbf{R}$ ).

Recentemente tomamos conhecimento da existência de dois artigos, relacionados ao estudo do problema restrito dos três corpos, nos quais é estudada a existência de pontos homoclínicos transversais de variedades invariantes de órbitas periódicas no infinito [12]. [30]. As técnicas utilizadas são distintas das apresentadas aqui. Além disso, os pontos críticos no infinito, considerados nos referidos trabalhos. são pontos de sela degenerados.

Existem muitos trabalhos sobre o estudo de órbitas periódicas subharmônicas para sistemas de equações diferenciais com perturbações periódicas (ver, por exemplo, [1], [2], [4], [5], [8], [15], [26], [28], [29]). O estudo de soluções subharmônicas aparece, em geral, em muitos tipos de aplicações físicas, como no caso das equações do pêndulo, de Van der Pol e de Duffing, com termo forçante periódico. Destaca-se também o interesse pela existência de órbitas subharmônicas no estudo da estabilidade do sistema solar, 
relacionada às órbitas dos planetas, satélites e asteróides.

Acreditamos que o estudo desenvolvido no Capítulo 4, sobre as bifurcações subharmônicas de grande amplitude, pode vir a contribuir no estudo de problemas aplicados. Embora tenha sido considerada uma perturbação um tanto particular na análise desenvolvido aqui, as técnicas utilizadas podem ser estendidas ao estudo de outros tipos de perturbações periódicas de campos polinomiais, conforme o interesse. 


\section{Referências Bibliográficas}

[1] K.T. Alligood, T.D. Sauer, and J.A. Yorke. CHAOS - An Introduction to Dynamical Systems. Springer Verlag New York, 1997.

[2] H. Bai-Lin. Chaos II. World Scientific, Singapore, 1990.

[3] S. Chandrasekhar. An Introduction to the Study of Stellar Structure. Dover, New York, 1967.

[4] C. Chicone. Bifurcations of nonlinear oscillations and frequency entrainment near resonance. SIAM J. Math. Anal., 23(6):157T-1608, 1992.

[5] C. Chicone. Ordinary Differential Equations with Applications. Texts in Appl. Math., 34. Springer-Verlag, New York, 1999.

[6] C. Chicone and F. Dumortier. Finiteness for critical periods of planar analytic vector fields. Nonlinear Analysis, Methods and Applications, 20(4):315-335, 1993.

[7] C. Chicone and J. Sotomayor. On a class of complete polynomial vector fields in the plane. Journal of Differential Equations, 61(3):398-418, 1986.

[8] S.N. Chow, J. Hale, and J. Mallet-Paret. An example of bifurcation to homoclinic orbits. Journal of Differential Equations, (37):351-373, 1980.

[9] C. Christopher. Quadratic systems having a parabola as an invariant curve. Proceedings of the Royal Society of Edingurgh, (112. ):113-134. 1989. 
[10] W.A. Coppel. A survey of quadratic systems. Journal of Differential Equations, (2):293-304, 1966.

[11] P. Cvitanovic. Universality in Chaos. Institute of Physics Publishing, London, 1989.

[12] H. Dankowicz and P. Holmes. The existence of transverse homoclinic points in the Sitnikov problem. Journal of Differential Equations, (116):468-483, 1995.

[13] S.P. Diliberto. On systems of ordinary differential equations. Annals of Mathematical Studies, 20:1-38, 1950. In Contributions to the Theory of Nonlinear Oscilations.

[14] D.G. Figueiredo and A.F. Neves. Equações Diferenciais Aplicadas. Coleção Matemática Universitária. IMPA/CXPq, 1997.

[15] J. Guckenheimer and P. Holmes. Nonlinear Oscilations, Dynamical Systems, and Bifurcations of Vector Fields. Appl. Math. Sciences, 42. Springer-Verlag, New York, 1983.

[16] J. Hale and P.Z. Táboas. Interaction of damping and forcing in a second order equation. Nonlinear Analysis, Theory, Methods and Applications, 2(1):77-84, 1978.

[1i] P. Hartman. Ordinary Differential Equations. John Wiley and Sons, New York, 1964.

[18] M.W. Hirsch and S. Smale. Differential Equations. Dynamical Systems and Linear Algebra. Academic Press, New York, 1974.

[19] X. Jarque and J. Llibre. Structural stability of planar hamiltonian polynomial vector fields. Proc. London Math. Soc., 68:617-640, 1964.

[20] V.K. Melnikov. On the stability of the center for time periodic perturbations. Trans. Moscow. Math. Soc., 12:1-5i, 1963. 
[21] H. Poincaré. Mémoire sur les courbes définies par une équation différentielle. $J$. Matématiques, 7:375-422, 1881.

[22] L. Shilnikov. Mathematical problems of nonlinear dynamics: a tutorial. Int. Journal of Bifurcation and Chaos, 7(9):1953-2001, 1997.

[23] J. Sotomayor. Estabilidade Estrutural de Primeira Ordem $\epsilon$ Variedades de Banach. Tese de Doutorado - IMPA, 1964.

[24] J. Sotomayor. Curvas Definidas por Equaçôes Diferenciais no Plano. IMPA-CNPq, 1981.

[25] J. Sotomayor and R. Paterlini. Bifurcations of polynomial vector fields in the plane. Canadian Mathematical Society - Conference Procєtdings. \$:665-685, 1987.

[26] P. Táboas. Periodic solutions of a forced Lotka-Volterra equation. Journal of Mathematical Analysis and Applications, (124):82-9ૉ, 1987.

[27] E.G. Velasco. Generic properties of polynomial vector fields at infinity. Amer. Math. Soc., 143, 1969.

[28] S. Wiggins. Global Bifurcations and Chaos - Analytical Methods. Applied Mathematical Sciences, 73. Springer-Verlag, New York, 1988.

[29] S. Wiggins. Introduction to Applied Nonlinear Dynamical Systems and Chaos. Texts in Applied Mathematics, 2. Springer-Verlag, New York, 1990.

[30] Z. Xia. Melnikov method and transversal homoclinic points in the restricted threebody problem. Journal of Differential Equations, 96:170-184, 1992. 